\title{
THE MEMBRANE THEORY OF SHELLS OF REVOLUTION
}

\author{
BY \\ C. TRUESDELL(")
}

Contents

I. METHODS AND CONCEPTS

1. The mathematical analysis of the statics of shells................. 97

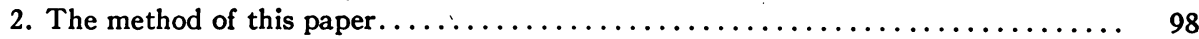

3. Revolution coordinates............................... 98

4. Stress resultants and moments. ............................ 99

II. Conical shells

5. The equations of the bending theory for conical shells............... 102

6. The equations of the membrane theory, and their integrals............. 109

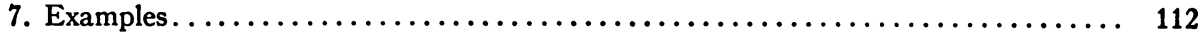

III. The DIfFERENTIAL EQUATIONS OF THE MEMBRANE THEORY FOR AN ARBITRARY SHELL OF REVOLUTION

8. The differential equations of the bending theory $\ldots \ldots \ldots \ldots \ldots \ldots \ldots \ldots \ldots \ldots$

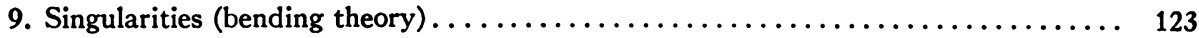

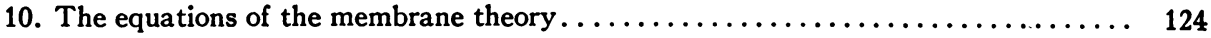

11. Stress functions. . . . . . . . . . . . . . . . . . 126

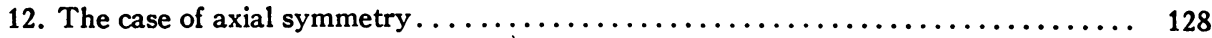

13. An example comparing the three-dimensional theory and the membrane theory.... 129

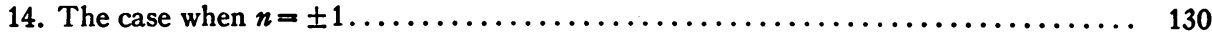

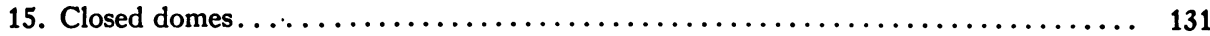

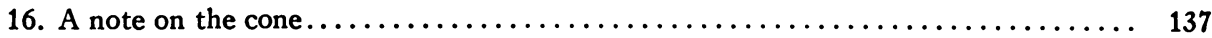

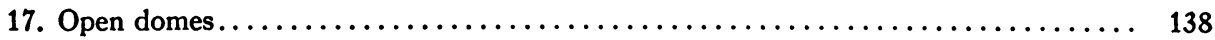

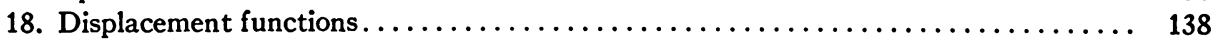

IV. EXamples

19. An indirect method of solution of the stress equation $\ldots \ldots \ldots \ldots \ldots \ldots \ldots \ldots \ldots 14$

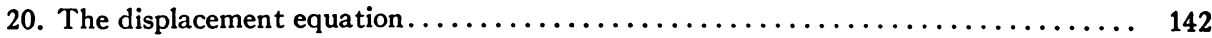

21. Bessel and exponential functions; paraboloids $\ldots \ldots \ldots \ldots \ldots \ldots \ldots \ldots \ldots \ldots, 143$

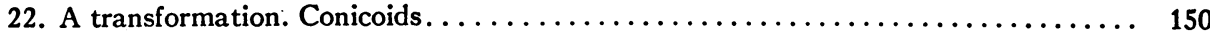

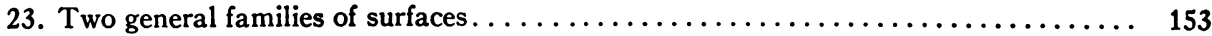

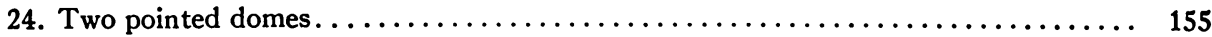

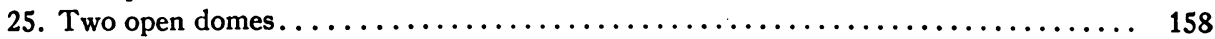

26. Two domes with a point of inflection in the meridian $\ldots \ldots \ldots \ldots \ldots \ldots \ldots \ldots \ldots 16 \ldots$

27. Stress functions for a few other surfaces....................... 163

Presented to the Society, September 13,1943, and February 26, 1945; received by the editors April 21, 1944.

(1) I began these investigations while an assistant at the School of Mathematical Mechanics, Brown University, in the summer of 1942. Much of the material in this paper formed a part of my Princeton doctoral thesis (1943). I wish to thank Professors Bateman, Bohnenblust, Lefschetz, Prager, E. Reissner, H. Reissner, and Tukey, and especially Professor Nemenyi, for reading and criticizing portions of these results in earlier versions. 
28. Nemenyi's integral equation $\ldots \ldots \ldots \ldots \ldots \ldots \ldots \ldots \ldots \ldots \ldots \ldots \ldots \ldots, 164$

Index of definitions and notations $\ldots \ldots \ldots \ldots \ldots \ldots \ldots \ldots \ldots \ldots \ldots, 165$

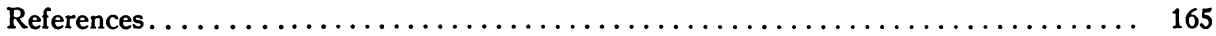

\section{Methods AND CONCEPTS}

1. The mathematical analysis of the statics of shells $\left({ }^{2}\right)$. It is possible to treat the equilibrium of a thin shell as a problem of three-dimensional elasticity. The boundary conditions are usually difficult to satisfy and the solutions are complicated, but there are cases of success, a notable one being given by Lamé( $\left.{ }^{3}\right)$ for a shell of any thickness bounded by arbitrarily loaded or displaced concentric spheres. When the shell is thin the variation of stress across it is often not particularly interesting, and a simpler but more enlightening description may be constructed in terms of the resultant forces and moments per unit length across a normal section; the theory of this description, under some special assumptions about the nature of the displacements produced, is called the bending theory of thin shells. For portions of the shell far from supports or points where one of the radii of normal curvature is small, the moments and certain other terms are negligible in comparison with the resultant stresses; the theory resulting when the former quantities are neglected is called the membrane theory of thin shells. We shall but touch on the bending theory to aid us in clarifying our notions; our concern in this paper is the membrane theory of shells whose middle section is initially a surface of revolution.

The raison d'être of the membrane theory is its simplicity in contrast to the bending theory, which, so far as I know, has not been forced to yield practical results except for the sphere, the circular cylinder, and the cone $\left(^{4}\right)$. In particular, the bending theory offers difficulties, as far as practical solutions are concerned, even for simple surfaces, when the load or support is not axially symmetric $\left({ }^{5}\right)$, although these difficulties have been overcome in some cases. Even when the solution given by the membrane theory is not adequate, it is customary to use it as a first approximation to which correcting terms from the bending theory are added. Even though the membrane theory is much simpler than the bending theory, except in cases of axial symmetry it has rarely been treated with success $\left({ }^{6}\right)$. The purpose of this paper is to supplement Flügge's excellent treatise, Statik und Dynamik der Schalen, in its treat-

(2) For a brief history of the investigations of shells, see Love (1927) pp. 5, 29. References are to the list at the end of the paper.

(8) Lamé (1854).

(4) Flüıge (1934), chaps. 6, 7; Timoshenko (1940), chap. 10; Geckeler (1929), pp. 238-260; Love (1927), pp. 565-613.

(5) Flügge (1934), p. 147.

( $)$ For a discussion of the treatment of unsymmetrical problems in the membrane theory previous to this work, see $\$ 11$, next to last paragraph. 
ment of the membrane theory of shells of revolution by giving an easy and practical method of exact solution of all the equations concerned, and by using this method of solution to discuss the limitations and capabilities of the membrane theory in general.

2. The method of this paper. I have not attempted to replace any portion of Flügge's book in its entirety, and hence I have not repeated the results of that work except when it seemed advisable to preserve the continuity of development; with a few exceptions, mostly in Chapter II, this paper contains new results (or new derivations) only $\left({ }^{7}\right)$. On the other hand, it is self-contained; the reader will require a knowledge of the fundamental notions of three-dimensional elasticity and of power series solutions of linear differential equations, but nothing of the theory of shells.

Since a shell is a three-dimensional body, and since the equations of threedimensional elasticity are well established, I think it preferable to derive the equations of shell theory as consequences of the general equations of elasticity rather than to follow the usual practice of deriving them from figures $\left.{ }^{8}\right)$.

The two surfaces of revolution for which the equations can be solved by quadratures without the use of Fourier series, namely the cone and the circular cylinder, are conveniently excluded from the general discussions of the theory; a complete treatment of cylinders of all types has been given by Flügge $\left({ }^{9}\right)$, while in the next chapter I give a discussion of the cone, which should serve to acquaint the reader with the characteristic advantages and defects of the membrane theory without introducing the complications of calculation which are the chief obstacle in the general theory which follows.

3. Revolution coordinates. We shall find it convenient to describe a thin shell in terms of a reference surface, called the middle surface, on which there are any convenient coordinates $\alpha^{1}, \alpha^{2}$. To obtain a three-dimensional coordinate system we erect normals at each point of the middle surface and call the distance measured outward along these normals $x$; then sufficiently near to regular points of the middle surface $\alpha^{1}, \alpha^{2}, x$ are suitable coordinates $\left({ }^{10}\right)$. $x=+\delta / 2$ and $x=-\delta / 2$, where $\delta=\delta\left(\alpha^{1}, \alpha^{2}\right)$, are the equations of two other surfaces equally distant from the middle surface. By a thin shell we mean the region between $x=+\delta / 2$ and $x=-\delta / 2$, if, except perhaps at certain singular points, $|\delta / R| \ll 1$, where $R$ is the minimum radius of normal curvature of the middle surface. $\delta$ is called the thickness of the shell. A point where one of the radii of normal curvature of the middle surfaces vanishes or becomes infinite will be called a singular point or singularity of the shell.

Throughout this work we shall be interested only in the case when the

(7) In particular, the sections on the membrane theory of shells of revolution in Flügge's book to which I have nothing to add are pp. 25-37, pp. 58-66.

$\left.{ }^{8}\right)$ For a note on the different derivations of the equations, see $\S 8$, footnote 27 .

(9) Flügge (1934), chap. 3.

(10) Such systems are those called "normal" by Synge and Chien (1941), p. 109. 
middle surface is a surface of revolution. We shall always use meridians and parallels as coordinate curves upon it $\left.{ }^{11}\right)$, and the corresponding three-dimensional coordinate system we call revolution coordinates. If we use the longitude angle $\theta$ as a parameter for the meridians, if $\phi$ is the colatitude angle, $r_{2}$ the distance along the normal to the surface to the axis of revolution, $r_{1}$ the radius of curvature of the meridian on the middle surface, $d m$ the element of arc

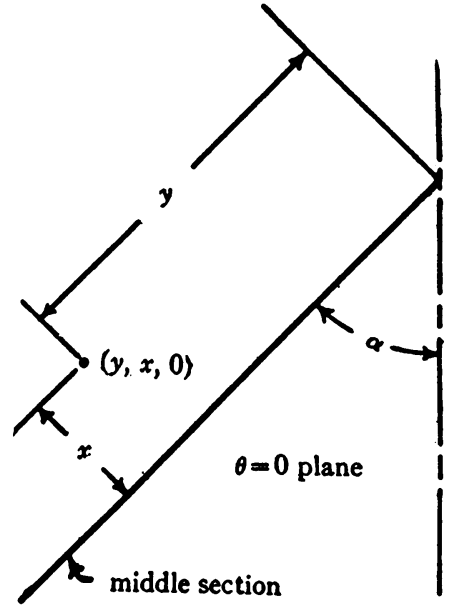

Fig. 1. Conical Coordinates.

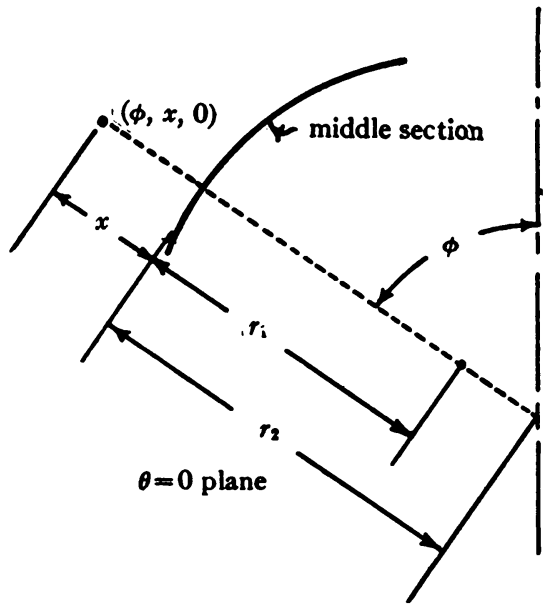

Fig. 2. Revolution Coordinates.

length on this meridian, then the element of arc length in revolution coordinates is defined by the equation( ${ }^{12}$ )

$$
d s^{2}=\left(\frac{r_{1}+x}{r_{1}}\right)^{2} d m^{2}+\left(r_{2}+x\right)^{2} \sin ^{2} \phi d \theta^{2}+d x^{2} .
$$

At a singularity of the shell, the element of arc length is undefined. If the middle section is a cone, it is convenient to parametrize the parallels by the distance $y$ along the meridian from the apex; formula (1) becomes, if $\alpha \equiv \pi / 2-\phi$,

$$
d s^{2}=d y^{2}+(y \sin \alpha+x \cos \alpha)^{2} d \theta^{2}+d x^{2} .
$$

Such a coordinate system we call conical coordinates (see Fig. 1). If the middle section is not a cone or cylinder, it is convenient to use $\phi$ as a parameter for the parallels, the element of arc length becoming then (see Fig. 2)

$$
d s^{2}=\left(r_{1}+x\right)^{2} d \phi^{2}+\left(r_{2}+x\right)^{2} \sin ^{2} \phi d \theta^{2}+d x^{2} .
$$

4. Stress resultants and moments. For three-dimensional bodies we are

(ii) To parallel the succeeding treatment for shells whose middle surface is not a surface of revolution one should use the lines of curvature as coordinate curves, as does E. Reissner (1941).

(12) In succeeding sections we refer to this formula as (3.1), and so on. 
accustomed to describe the distribution of forces by means of the stresses $\tau_{i j}$ (physical components) giving components of the force per unit area across a surface. For thin shells one can obtain a simpler and more enlightening picture by introducing stress-resultants, quantities defined on coordinate curves on the middle surface which when integrated along such a curve give the same total pressure as do the stresses integrated over a normal section of the shell cut along the curve. Thus, for example, the stress resultant $N_{\phi}$ in the $\phi$ direction across a $\phi=$ const. curve is defined by the relation

$$
\int_{\theta_{0}}^{\theta} N_{\phi} \cdot r_{2} \sin \phi d \theta=\int_{\theta_{0}}^{\theta} \int_{-\delta / 2}^{+\delta / 2} \tau_{\phi \phi} \cdot\left(r_{2}+x\right) \sin \phi d \theta d x .
$$

Hence, by differentiation,

$$
N_{\phi}=\int_{-\delta / 2}^{+\delta / 2} \tau_{\phi \phi}\left(1+\frac{x}{r_{2}}\right) d x .
$$

Resultant moments are similarly defined. In all, we have, in the conventional notation, ten resultants $\left({ }^{13}\right)$ :

$$
\begin{array}{rlrl}
N_{\phi} & =\int_{-\delta / 2}^{+\delta / 2} \tau_{\phi \phi}\left(1+\frac{x}{r_{2}}\right) d x, & N_{\theta}=\int_{-\delta / 2}^{+\delta / 2} \tau_{\theta \theta}\left(1+\frac{x}{r_{1}}\right) d x, \\
N_{\phi \theta}=\int_{-\delta / 2}^{+\delta / 2} \tau_{\theta \phi}\left(1+\frac{x}{r_{2}}\right) d x, & N_{\theta \phi}=\int_{-\delta / 2}^{+\delta / 2} \tau_{\phi \theta}\left(1+\frac{x}{r_{1}}\right) d x \\
\text { (1) } Q_{\phi}=-\int_{-\delta / 2}^{+\delta / 2} \tau_{\phi x}\left(1+\frac{x}{r_{2}}\right) d x, & Q_{\theta}=-\int_{-\delta / 2}^{+\delta / 2} \tau_{\theta x}\left(1+\frac{x}{r_{1}}\right) d x \\
M_{\phi}=-\int_{-\delta / 2}^{+\delta / 2} x \tau_{\phi \phi}\left(1+\frac{x}{r_{2}}\right) d x, & M_{\theta}=-\int_{-\delta / 2}^{+\delta / 2} x \tau_{\theta \theta}\left(1+\frac{x}{r_{1}}\right) d x \\
M_{\phi \theta}=-\int_{-\delta / 2}^{+\delta / 2} x \tau_{\phi \theta}\left(1+\frac{x}{r_{2}}\right) d x, & M_{\theta \phi}=-\int_{-\delta / 2}^{+\delta / 2} x \tau_{\theta \phi}\left(1+\frac{x}{r_{1}}\right) d x
\end{array}
$$

The moments $M_{\phi}$ and $M_{\theta}$ tend to bend the meridians and parallels, respectively, $M_{\phi \theta}$ and $M_{\theta \phi}$ to twist them.

While these components are not symmetric, the symmetry of the stress tensor implies the relation

$$
\begin{aligned}
\tau_{\theta \phi}\left(1+\frac{x}{r_{1}}\right)-\tau_{\phi \theta}\left(1+\frac{x}{r_{2}}\right)=-\frac{1}{r_{2}} & {\left[x \tau_{\theta \phi}\left(1+\frac{x}{r_{1}}\right)\right] } \\
& +\frac{1}{r_{1}}\left[x \tau_{\phi \theta}\left(1+\frac{x}{r_{2}}\right)\right]
\end{aligned}
$$

(13) These are the components of the "macroscopic force and bending tensors" of Synge and Chien (1941). They were introduced by Love in 1888 . 
integrating, we find that

$$
N_{\theta \phi}-N_{\phi \theta}=\frac{M_{\theta \phi}}{r_{2}}-\frac{M_{\phi \theta}}{r_{1}} .
$$

Body force $\left(\phi_{x}, \phi_{\phi}, \phi_{\theta}\right)$ (physical components) we may replace by an equivalent force per unit area acting on the middle surface:

$$
\begin{aligned}
& Z_{b}=-\int_{-\delta / 2}^{+\delta / 2} \phi_{x}\left(1+\frac{x}{r_{1}}\right)\left(1+\frac{x}{r_{2}}\right) d x, \\
& Y_{b}=\int_{-\delta / 2}^{+\delta / 2} \phi_{\phi}\left(1+\frac{x}{r_{1}}\right)\left(1+\frac{x}{r_{2}}\right) d x, \\
& X_{b}=\int_{-\delta / 2}^{+\delta / 2} \phi_{\theta}\left(1+\frac{x}{r_{1}}\right)\left(1+\frac{x}{r_{2}}\right) d x,
\end{aligned}
$$

while surface loads $\left(Z_{o}, Y_{o}, X_{o}\right)$ and $\left(Z_{i}, Y_{i}, X_{i}\right)$ in the $-x, \phi, \theta$ directions respectively on the outer and inner surfaces may be replaced by equivalent surface forces acting on the middle section:

$$
\begin{aligned}
& Z_{s}=\left(1+\frac{\delta}{2 r_{1}}\right)\left(1+\frac{\delta}{2 r_{2}}\right) Z_{o}-\left(1-\frac{\delta}{2 r_{1}}\right)\left(1-\frac{\delta}{2 r_{2}}\right) Z_{i}, \\
& Y_{c}=\left(1+\frac{\delta}{2 r_{1}}\right)\left(1+\frac{\delta}{2 r_{2}}\right) Y_{o}-\left(1-\frac{\delta}{2 r_{1}}\right)\left(1-\frac{\delta}{2 r_{2}}\right) Y_{i}, \\
& X_{c}=\left(1+\frac{\delta}{2 r_{1}}\right)\left(1+\frac{\delta}{2 r_{2}}\right) X_{0}-\left(1-\frac{\delta}{2 r_{1}}\right)\left(1-\frac{\delta}{2 r_{2}}\right) X_{i} .
\end{aligned}
$$

Let $\left(Z_{t}, Y_{t}, X_{t}\right)$ be the resultant force per unit area of the middle section due to the load :

$$
Z_{t}=Z_{b}+Z_{s}, \quad Y_{t}=Y_{b}+Y_{a}, \quad X_{t}=X_{b}+X_{\bullet} .
$$

Then the combined load on the shell may be represented by this equivalent force density, provided we add a suitable moment density $\left(L_{y}, L_{x}\right)$ :

(6)

$$
\begin{aligned}
L_{y}= & -\int_{-\delta / 2}^{+\delta / 2} x \phi_{\phi}\left(1+\frac{x}{r_{1}}\right)\left(1+\frac{x}{r_{2}}\right) d x \\
& -\frac{\delta}{2}\left[\left(1+\frac{\delta}{2 r_{1}}\right)\left(1+\frac{\delta}{2 r_{2}}\right) Y_{0}+\left(1-\frac{\delta}{2 r_{1}}\right)\left(1-\frac{\delta}{2 r_{2}}\right) Y_{i}\right], \\
L_{x}= & -\int_{-\delta / 2}^{+\delta / 2} x \phi_{\theta}\left(1+\frac{x}{r_{1}}\right)\left(1+\frac{x}{r_{2}}\right) d x \\
& -\frac{\delta}{2}\left[\left(1+\frac{\delta}{2 r_{1}}\right)\left(1+\frac{\delta}{2 r_{2}}\right) X_{0}+\left(1-\frac{\delta}{2 r_{1}}\right)\left(1-\frac{\delta}{2 r_{2}}\right) X_{i}\right] .
\end{aligned}
$$


All these definitions, which have been phrased for an element of arc length (3.3) in revolution coordinates, can be transferred to conical coordinates with element of arc length (3.2) if we replace $r_{1}$ by $\infty, \phi$ by $y$, and $r_{2}$ by $y \tan \alpha$ respectively.

From the usual sort of equilibrium considerations it is easy to show that the resultants transform according to the tensor law under orthogonal transformations.

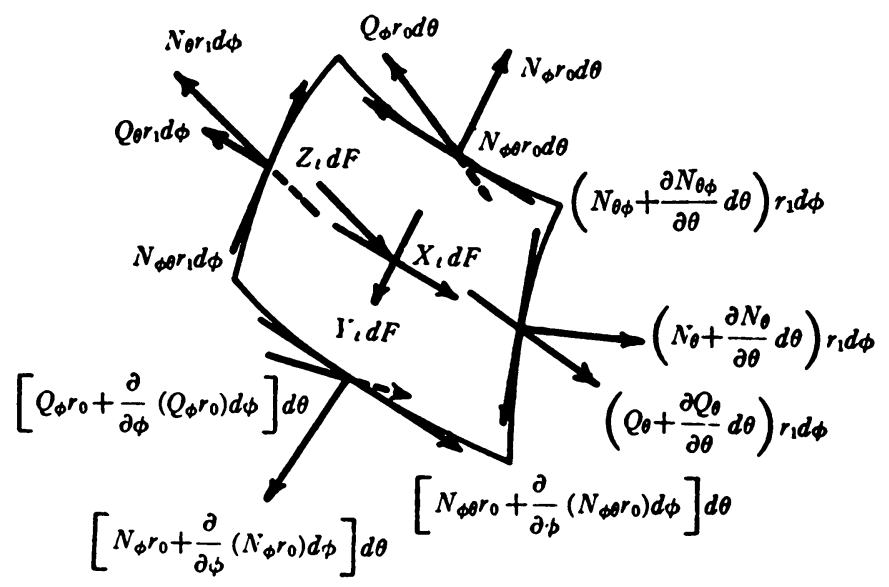

FIG. 3a.

Stress resultants and load force density.

A visualization of the forces and moments acting on a small section $d F$ of the shell may be seen in Fig. 3.

\section{Conical shells}

5. The equations of the bending theory for conical shells. Let the threedimensional stresses and strains (physical components) be $\tau_{x x}, \cdots, \epsilon_{x x}, \cdots$, the physical components of body force be $\phi_{x}, \ldots$. Then the equilibrium equations of three-dimensional elasticity $\left({ }^{14}\right)$ written in conical coordinates with element of arc length (3.2) are

$$
\frac{\partial}{\partial y}\left[(y \sin \alpha+x \cos \alpha) \tau_{y y}\right]-\tau_{\theta \theta} \sin \alpha+\frac{\partial \tau_{y \theta}}{\partial \theta}
$$

$$
+\frac{\partial}{\partial x}\left[(y \sin \alpha+x \cos \alpha) \tau_{y x}\right]+(y \sin \alpha+x \cos \alpha) \phi_{y}=0,
$$

(14) Love (1927), p. 90; Trefftz (1928), p. 81; Sokolnikoff (1941), p. 179. 
$\frac{\partial}{\partial y}\left[(y \sin \alpha+x \cos \alpha) \tau_{y \theta}\right]+\tau_{y \theta} \sin \alpha+\frac{\partial \tau_{\theta \theta}}{\partial \theta}$

$$
+\frac{\partial}{\partial x}\left[(y \sin \alpha+x \cos \alpha) \tau_{x \theta}\right]+\tau_{x \theta} \cos \alpha+(y \sin \alpha+x \cos \alpha) \phi_{\theta}=0,
$$

$\frac{\partial}{\partial x}\left[(y \sin \alpha+x \cos \alpha) \tau_{x x}\right]+\frac{\partial}{\partial y}\left[(y \sin \alpha+x \cos \alpha) \tau_{x y}\right]$

$$
+\frac{\partial \tau_{x \theta}}{\partial \theta}-\tau_{\theta \theta} \cos \alpha+(y \sin \alpha+x \cos \alpha) \phi_{x}=0 .
$$

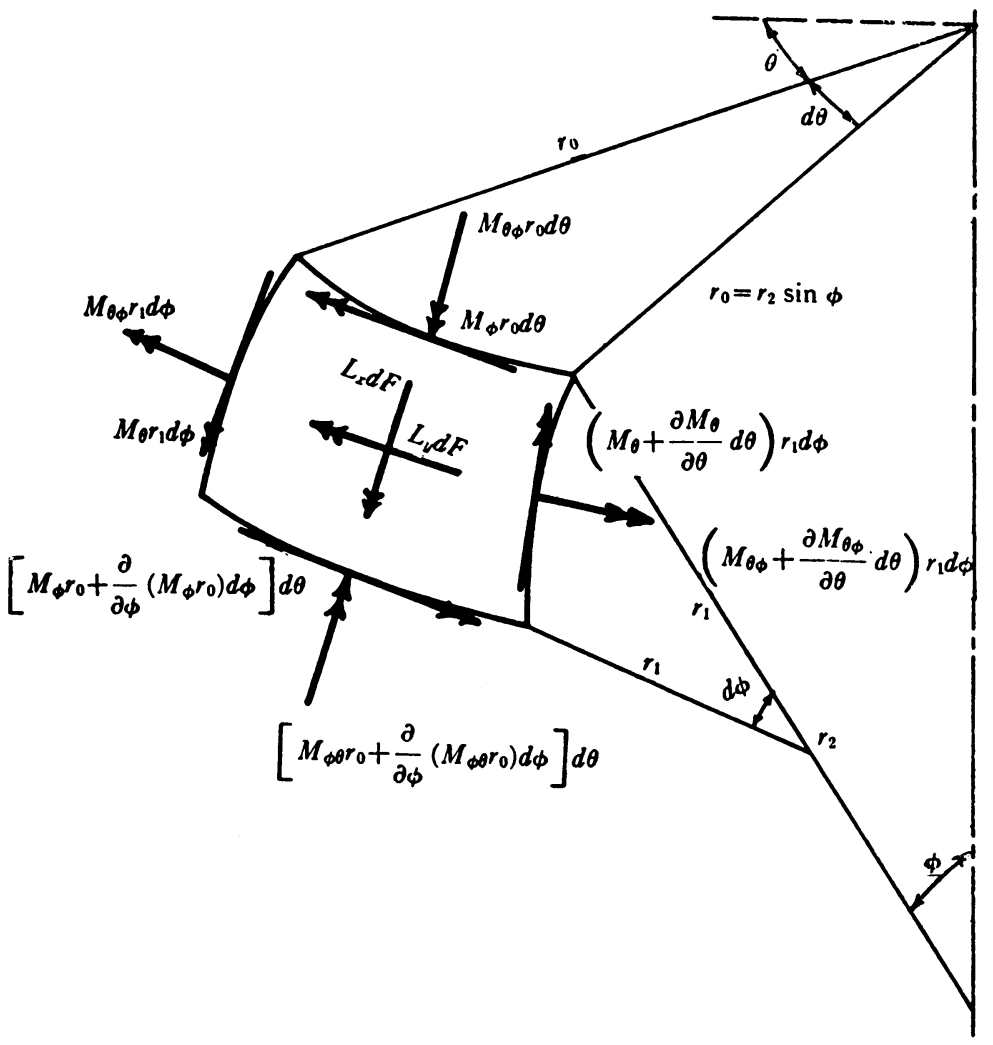

FIG. 3b.

Moment resultants and load moment density.

To these equations we add Hooke's law connecting the stresses and strains $\left({ }^{(15)}\right.$

(15) Love (1927), pp. 102-103; Trefftz (1928), pp. 61-62; Sokolnikoff (1941), p. 179. 
and the conditions of compatibility $\left({ }^{10}\right)$. We shall suppose there exist unique functions $\tau_{x x}, \cdots, \epsilon_{x x}, \cdots$ satisfying all these equations, such that on $x=+\delta / 2$,

$$
\tau_{x x}=-Z_{o}, \quad \tau_{x y}=Y_{o}, \quad \tau_{x \theta}=X_{o},
$$

and on $x=-\delta / 2$,

$$
\tau_{x x}=-Z_{i}, \quad \tau_{x y}=Y_{i}, \quad \tau_{x \theta}=X_{i},
$$

while on some boundary (or boundaries) $y=$ const., $\tau_{y x}, \tau_{y \theta}$, and $\tau_{y y}$ assume prescribed values. If we integrate the equations (1) with respect to $x$ from $-\delta / 2$ to $+\delta / 2$, using the definitions (4.1) and (4.5), we obtain three equilibrium equations in terms of the resultants :

$$
\begin{array}{r}
\frac{\partial N_{y}}{\partial y}+\frac{1}{y}\left(N_{y}-N_{\theta}\right)+\frac{\csc \alpha}{y} \frac{\partial N_{\theta y}}{\partial y}+Y_{t}=0 \\
\frac{\partial N_{y \theta}}{\partial y}+\frac{1}{y}\left(N_{y \theta}+N_{\theta y}\right)+\frac{\csc \alpha}{y} \frac{\partial N_{\theta}}{\partial \theta}-\frac{\cot \alpha}{y} Q_{\theta}+X_{t}=0 \\
\sin \alpha \frac{\partial}{\partial y}\left(y Q_{y}\right)+\frac{\partial Q_{\theta}}{\partial \theta}+N_{\theta} \cos \alpha+y \sin \alpha Z_{t}=0 .
\end{array}
$$

If we multiply the first and second of equations (1) by $x$ and integrate from $-\delta / 2$ to $+\delta / 2$, using (4.1) and (4.6), we obtain two more equilibrium conditions:

$$
\begin{aligned}
& \frac{\partial}{\partial y}\left(y M_{y}\right)-M_{\theta}+\csc \alpha \frac{\partial M_{\theta y}}{\partial \theta}-y Q_{y}+y L_{y}=0, \\
& \frac{\partial}{\partial y}\left(y M_{y \theta}\right)-M_{\theta y}+\csc \alpha \frac{\partial M_{\theta}}{\partial \theta}-y Q_{\theta}+y L_{x}=0 .
\end{aligned}
$$

The conditions satisfied on boundaries $y=$ const. can be translated by the definitions (4.1) into boundary conditions on the solutions of the systems (2) and (3). While we now have five equilibrium equations in ten resultants and moments instead of three equations in six stresses, the new system is much easier to handle because the boundary conditions have been reduced by six:

In order to obtain a manageable description of the deformation, we agree to treat only those problems which lead to displacements having the following properties $\left({ }^{17}\right)$ :

$$
\begin{aligned}
& \text { (I) }\left|\epsilon_{x y}\right| \ll \delta / R,\left|\epsilon_{x \theta}\right| \ll \delta / R,\left|\epsilon_{x x}\right| \ll \delta / R,\left|R \frac{\partial \epsilon_{x x}}{\partial y}\right| \ll 1,\left|\frac{\partial \epsilon_{x x}}{\partial \theta}\right| \ll 1, \\
& \text { (II) }\left|\tau_{x x} / E\right| \ll\left|\epsilon_{x x}+\nu \Delta /(1-\nu)\right|
\end{aligned}
$$

(10) Odqvist (1937).

(17) These are essentially the assumptions of E. Reissner (1941). 
where $R=y \tan \alpha, \Delta$ is the cubical dilatation, $E$ is Young's modulus, and $\nu$ is Poisson's ratio. Assumption (I) states that a line initially normal to the midsection is deformed into a line essentially normal to the deformed mid-section, and that the thickness $\delta$ is great enough that if $\Delta \delta$ is the change in $\delta$ due to the deformation, then $|\Delta \delta| \ll \delta^{2} / R$. Assumption (II) does not state that $\tau_{x x}$ is negligible in the equilibrium considerations, but that the contribution of the dimensionless ratio $\tau_{x x} / E$ to the deformation is negligible. As a consequence, from Hooke's law we see that, approximately,

$$
\epsilon_{x x}=-\nu \Delta /(1-\nu) \text {; }
$$

this equation implies, by Assumption (I), that $|\Delta| \ll \delta / R$, that is, the cubical dilatation is much smaller than the relative thickness, and that, since $\nu \approx 1 / 3$ for most structural materials, $\epsilon_{x x} \approx\left(\epsilon_{y y}+\epsilon_{\theta \theta}\right) / 2$, or that the normal deformation is the negative of the mean tangential deformation, and that, in particular, deformations in which the shell is compressed or extended both in area and thickness are excluded. These assumptions are such as to exclude very thin or very thick shells, and to make the displacements of points on the middle surface a sufficiently accurate indication of the distribution of displacements throughout the shell, as we shall see shortly.

By using (4) we may simplify the remaining equations of Hooke's law, three of which become:

$$
\begin{gathered}
\tau_{\nu y}=\left(E /\left(1-\nu^{2}\right)\right)\left(\epsilon_{y y}+\nu \epsilon_{\theta \theta}\right), \quad \tau_{\theta \theta}=\left(E /\left(1-\nu^{2}\right)\right)\left(\epsilon_{\theta \theta}+\nu \epsilon_{y y}\right), \\
\tau_{\nu \theta}=E \epsilon_{y \theta} /(1+\nu) .
\end{gathered}
$$

We shall not need to use the last two equations of Hooke's law, so I do not write them here.

Let the displacements of a point in the $\theta, y, x$ directions be denoted by $u, v, w$ respectively. Then the expressions giving the strains in terms of the displacements become $\left({ }^{18}\right)$

$$
\epsilon_{y y}=\frac{\partial v}{\partial y}, \quad \epsilon_{\theta \theta}=\frac{\frac{\partial u}{\partial \theta}+v \sin \alpha+w \cos \alpha}{y \sin \alpha+x \cos \alpha}, \quad \epsilon_{x x}=\frac{\partial w}{\partial x},
$$

$$
\begin{aligned}
\epsilon_{y \theta} & =\frac{\frac{\partial v}{\partial \theta}+(y \sin \alpha+x \cos \alpha) \frac{\partial u}{\partial y}-u \sin \alpha}{2(y \sin \alpha+x \cos \alpha)}, \\
\epsilon_{x y} & =\left(\frac{\partial w}{\partial y}+\frac{\partial v}{\partial x}\right) / 2, \quad \epsilon_{x \theta}=\frac{(y \sin \alpha+x \cos \alpha) \frac{\partial u}{\partial x}-u \cos \alpha+\frac{\partial w}{\partial \theta}}{2(y \sin \alpha+x \cos \alpha)} .
\end{aligned}
$$

(18) Love (1927), p. 54; Trefftz (1928), p. 78; Sokolnikoff (1941), pp. 177-178. 
Let us now express the displacement $(u, v, w)$ of a point $(\theta, y, X)$ in terms of $X$, the displacement $(U, V, W)$ of its projection $(\theta, y, 0)$ on the middle surface, and the strains, using the equations (6). For example,

$$
\begin{aligned}
u & =U+\int_{0}^{x} \frac{\partial u}{\partial x} d x \\
& =U-\int_{0}^{x} \frac{u \cos \alpha-\frac{\partial w}{\partial \theta}}{y \sin \alpha+x \cos \alpha} d(X-x)+2 \int_{0}^{x} \epsilon_{x \theta} d x \\
& =U+X \frac{U \cos \alpha-\frac{\partial W}{\partial \theta}}{y \sin \alpha} \\
& \quad-\int_{0}^{x} \frac{X-x}{y \sin \alpha+x \cos \alpha}\left[2 \epsilon_{x \theta} \cos \alpha-\frac{\partial \epsilon_{x x}}{\partial \theta}\right] d x+2 \int_{0}^{x} \epsilon_{x \theta} d x .
\end{aligned}
$$

Rearranging this result, and carrying out a similar computation for $v$ and $w$, we obtain the formulas

$$
\begin{aligned}
& u=U+X\left[\frac{\cot \alpha}{y} U-\frac{\csc \alpha}{y} \frac{\partial W}{\partial \theta}\right] \\
& +\int_{0}^{x} \frac{2(y \sin \alpha-x \cos \alpha) \epsilon_{x \theta}+(X-x) \frac{\partial \epsilon_{x x}}{\partial \theta}}{y \sin \alpha+x \cos \alpha} d x, \\
& v=V-X \frac{\partial W}{\partial y}+\int_{0}^{x}\left[(X-x) \frac{\partial \epsilon_{x x}}{\partial y}+2 \epsilon_{x y}\right] d x, \\
& w=W+\int_{0}^{x} \epsilon_{x x} d x .
\end{aligned}
$$

Replacing $X$ by $x$, and retaining only terms of up to and including the second order in $x$, we find that under Assumptions (I) and (II) the relations (7) become simply

$$
\begin{aligned}
& u=U+\frac{x}{y}\left(U \cot \alpha-\csc \alpha \frac{\partial W}{\partial \theta}\right), \\
& v=V-x \frac{\partial W}{\partial y}, \\
& w=W .
\end{aligned}
$$

We can now put these expressions into the formulas (6) for the strains, put 
the resulting equations into Hooke's law (5), and evaluate the integrals (4.1) for the resultants and moments. We are justified in retaining terms in $x^{2}$ in the integrands; hence the resulting expressions are correct up to and including terms in $\delta^{4}$, since any terms in $x^{3}$ would vanish in the integration. We obtain the following formulas expressing the resultants and moments in terms of the displacements $U, V, W$ of the middle surface:

$$
\begin{aligned}
& N_{y}=D\left[\frac{\partial V}{\partial y}+\frac{\nu \csc \alpha}{y}\left(\frac{\partial U}{\partial \theta}+V \sin \alpha+W \cos \alpha\right)\right] \\
& -\frac{K}{y} \cot \alpha \frac{\partial^{2} W}{\partial y^{2}} \\
& N_{\theta}=D\left[\frac{\csc \alpha}{y}\left(\frac{\partial U}{\partial \theta}+V \sin \alpha+W \cos \alpha\right)+\nu \frac{\partial V}{\partial y}\right] \\
& +\frac{K \cot \alpha}{y^{8} \sin \alpha}\left[V \cos \alpha+W \cot \alpha \cos \alpha+\csc \alpha \frac{\partial^{2} W}{\partial \theta^{2}}+y \sin \alpha \frac{\partial W}{\partial y}\right] \text {, } \\
& N_{y \theta}=\frac{D(1-\nu)}{2 y \sin \alpha}\left[\frac{\partial V}{\partial \theta}+y \sin \alpha \frac{\partial U}{\partial y}-U \sin \alpha\right] \\
& +\frac{K(1-\nu) \cot \alpha}{2 y^{3} \sin \alpha}\left[y \cos \alpha \frac{\partial U}{\partial y}-U \cos \alpha-y \frac{\partial^{2} W}{\partial \theta \partial y}+\frac{\partial W}{\partial \theta}\right] \text {, } \\
& N_{\theta y}=\frac{D(1-\nu)}{2 y \sin \alpha}\left[\frac{\partial V}{\partial \theta}+y \sin \alpha \frac{\partial U}{\partial y}-U \sin \alpha\right] \\
& +\frac{K(1-\nu) \cot \alpha}{2 y^{3} \sin \alpha}\left[\cot \alpha \frac{\partial V}{\partial \theta}+y \frac{\partial^{2} W}{\partial \theta \partial y}-\frac{\partial W}{\partial \theta}\right], \\
& M_{y}=K\left[\frac{\partial^{2} W}{\partial y^{2}}-\frac{\cot \alpha}{y} \frac{\partial V}{\partial y}\right. \\
& \left.+\frac{\nu}{y}\left(\frac{\partial W}{\partial y}+\frac{\csc ^{2} \alpha}{y} \frac{\partial^{2} W}{\partial \theta^{2}}-\frac{\cot \alpha \csc \alpha}{y} \frac{\partial U}{\partial \theta}\right)\right] \text {, } \\
& M_{\theta}=K\left[\frac{1}{y^{2}}\left(V \cot \alpha+W \cot ^{2} \alpha+\csc ^{2} \alpha \frac{\partial^{2} W}{\partial \theta^{2}}+y \frac{\partial W}{\partial y}\right)+\nu \frac{\partial^{2} W}{\partial y^{2}}\right] \text {, } \\
& M_{y \theta}=\frac{K(1-\nu)}{y^{2} \sin \alpha}\left[U \cos \alpha-y \cos \alpha \frac{\partial U}{\partial y}+y \frac{\partial^{2} W}{\partial \theta \partial y}-\frac{\partial W}{\partial \theta}\right] \text {, } \\
& M_{\theta \nu}=\frac{K(1-\nu)}{2 y^{2} \sin \alpha}\left[\cot \alpha \frac{\partial V}{\partial \theta}-y \cos \alpha \frac{\partial U}{\partial y}\right. \\
& \left.+U \cos \alpha+2 y \frac{\partial^{2} W}{\partial \theta \partial y}-2 \frac{\partial W}{\partial \theta}\right] \text {, }
\end{aligned}
$$


where

$$
D \equiv E \delta /\left(1-\nu^{2}\right), \quad K \equiv E \delta^{3} /\left(12\left(1-\nu^{2}\right)\right) .
$$

$D$ and $K$ are called the flexural rigidity and the bending rigidity respectively. $E$, and hence also $D$ and $K$, has been assumed independent of $x$, but not necessarily of $y$ and $\theta$. Similar expressions could be written down for $Q_{\theta}$ and $Q_{\boldsymbol{y}}$, but we shall not need them. Of course, these formulas satisfy identically the relation (4.2), which in conical coordinates has the form

$$
N_{\theta y}-N_{y \theta}=\frac{\cot \alpha}{y} M_{\theta y} .
$$

Equations (2), (3), and (9) form a system of thirteen equations in thirteen unknowns, which can easily be reduced by elimination to a system of three equations in $U, V, W$ alone. These are the equations of the bending theory. If the three-dimensional solution of a problem in question produces a state of strain satisfying Assumptions (I) and (II), there will exist a solution of these equations, and this solution will be as correct as the corresponding threedimensional solution, provided we are justified in neglecting powers of $\delta /(y \tan \alpha)$ higher than the fourth. In this form the bending theory presents a rather complicated but completely correct picture of the behavior of a thin conical shell under small deformations with small strains satisfying Assumptions (I) and (II). If such a deformation is produced by given loads in a given shell, the bending theory equations will be correct. An estimate of the validity of the assumptions can be obtained by computing the values of the strains at the middle section by substituting the solutions $U, V, W$ in (6) and putting $x$ equal to zero; if these values of the strains do not satisfy Assumptions (I) and (II), we know the bending theory is inadequate to treat the problem in question. If these values of the strains do satisfy Assumptions (I) and (II) at the middle surface, we are reasonably sure the bending theory is adequate; if we were certain the strains did not vary much with $x$, we should be certain the bending theory was correct for the problem in question.

We have two boundaries $y=$ const. (one may be the apex, the other at infinity) at which three stress components, and hence five resultants and moments, may be prescribed. These ten quantities are subject to the six conditions of equilibrium of the entire body under all its loads. Hence we may prescribe four independent boundary conditions on the moments and resultants.

The formal difficulties of the bending theory are considerable unless further simplifying assumptions are made. Under conditions of complete symmetry with respect to $\theta$, when the shear resultants and moments vanish, the equations may be solved in terms of Bessel functions if $\delta$ is a constant $\left({ }^{10}\right)$,

(19) Flügge (1934), pp. 160-165; Timoshenko (1940), pp. 475-479. 
or with simpler functions if $\delta=k y$. Our concern with these equations, however, is only to clarify the nature of the membrane theory.

Before leaving the bending theory we should notice that the effect of the singularity at $x=0, y=0$ is that the resultants and moments should not be expected to have much meaning in its vicinity, since the integrals defining them are unaffected by the stress distribution in the portion of the region where $x>0$ and $y<0$ which lies in the cone (the shaded region in Fig. 4). The bending theory is not necessarily incorrect in this vicinity, but it does not furnish a satisfactory description of events. For an accurate picture we should

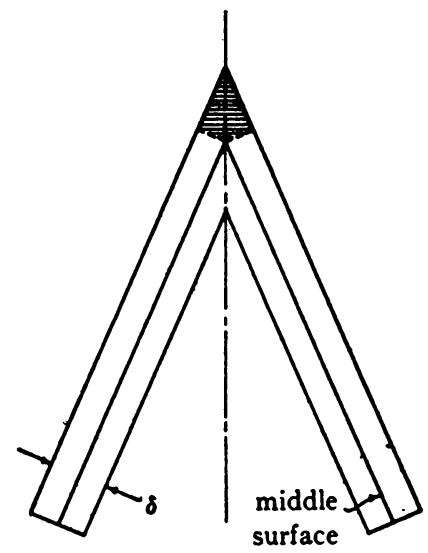

FIG. 4.

resort to the three-dimensional theory. Usually, however, the apex is not a point of interest. Since $\theta$ is undefined at the apex, so are $\tau_{\theta \theta}, \tau_{\theta y}$, and $\tau_{\theta x}$, so the equilibrium equations (1) cannot be expected to have a meaning there, and hence neither can the equations of the bending theory; we shall content ourselves for the present with the artificial requirement that all resultants and moments remain finite at the apex, the boundary condition customarily imposed. Further discussion of singularities we defer until $\S \S 9,15$.

6. The equations of the membrane theory, and their integrals. Since we are supposing that, except near $y=0, \delta /(y \tan \alpha) \ll 1$, it follows that $K /\left(y^{2} \tan ^{2} \alpha\right) \ll D$, and the terms in (5.9) of which $K$ is a coefficient may be neglected unless they are much larger than the terms of which $D$ is a coefficient. Under the assumptions of the classical theory of elasticity, that displacements and their derivatives are small, these terms will then be negligible except (i) near the apex, $y=0$, or (ii) when they are prescribed quantities at certain boundaries. When these quantities are negligible the shell is said to admit a state of membrane stress, and the equations of the bending theory are much simplified. All moments vanish, and $N_{y \theta}=N_{\theta y}$. Equations (5.3) give us the values of $Q_{\nu}$ and $Q_{0}$ at once: 
(1)

$$
Q_{y}=L_{y}, \quad Q_{\theta}=L_{x} .
$$

If we define quantities $Z, Y, X$ :

$$
\begin{aligned}
& Z \equiv Z_{t}+\frac{\csc \alpha}{y} \frac{\partial L_{x}}{\partial \theta}+\frac{1}{y} \frac{\partial}{\partial y}\left(y L_{y}\right), \\
& Y \equiv Y_{t}, \\
& X \equiv X_{t}-\frac{\cot \alpha}{y} L_{x},
\end{aligned}
$$

the remaining equilibrium equations (5.2) become simply

$$
\begin{gathered}
\frac{\partial N_{y}}{\partial y}+\frac{1}{y}\left(N_{y}-N_{\theta}\right)+\frac{\csc \alpha}{y} \frac{\partial N_{\theta y}}{\partial \theta}+Y=0, \\
\frac{\partial N_{\theta y}}{\partial y}+\frac{2}{y} N_{\theta y}+\frac{\csc \alpha}{y} \frac{\partial N_{\theta}}{\partial \theta}+X=0, \\
N_{\theta}=-y \tan \alpha Z .
\end{gathered}
$$

For most cases of interest, the load moments are negligible, and $(Z, Y, X)$ represents the equivalent force density of load on the middle section. The relations (5.9) which do not vanish may be rearranged into the form

$$
\frac{\partial V}{\partial y}=\frac{1}{E \delta}\left(N_{\nu}-\nu N_{\theta}\right),
$$

$$
\begin{aligned}
\frac{\partial U}{\partial \theta}+V \sin \alpha+W \cos \alpha & =\frac{y \sin \alpha}{E \delta}\left(N_{\theta}-\nu N_{y}\right), \\
\csc \alpha \frac{\partial V}{\partial \theta}+y \frac{\partial U}{\partial y}-U & =\frac{2 y(1+\nu)}{E \delta} N_{\theta y} .
\end{aligned}
$$

The equations (3) and (4) are the equations of the membrane theory of cones; like those of the bending theory, they form a determined system, from which by elimination we could derive three equations in the three unknowns $U, V, W$ alone.

When we have solved the equations of the membrane theory for a particular boundary problem, we can always tell whether the problem in question admits a membrane state of stress by substituting the displacements $U, V, W$ in the equations of the bending theory (5.9); if all the terms following $K$ are of the same magnitude as those following $D$, then we know that our solution of the membrane theory equations is a solution of the bending theory equations correct up to and including terms of the second order in $\delta /(y \tan \alpha)$. Thus except near the apex of a cone whose behavior is described by the bending theory, there is always a membrane state of stress unless the boundary 
conditions forbid it. It is possible, of course, that even near the apex there may be a membrane state of stress.

In the usual treatment of the theory Hooke's law is written in the form

$$
\epsilon_{y y}=\frac{1}{E \delta}\left(N_{y}-\nu N_{\theta}\right), \quad \epsilon_{\theta \theta}=\frac{1}{E \delta}\left(N_{\theta}-\nu N_{y}\right), \quad \epsilon_{\theta y}=\frac{1+\nu}{E \delta} N_{\theta y} .
$$

If $\epsilon_{y y}, \epsilon_{\theta \theta}, \epsilon_{\theta y}$ are regarded as approximations to the three-dimensional strains, the expressions (5) are incorrect; they contradict our earlier formulation of Hooke's law (5.5), and furthermore such strains cannot satisfy the conditions of compatibility, even approximately $\left({ }^{20}\right)$. The equations (5) give the correct values of the three-dimensional strains at the middle section, but it is incorrect to neglect their variation across the thickness of the shell. The conditions of compatibility may in fact be used, with the aid of equations (5), to compute the correct values of the derivatives of the strains with respect to $x$ evaluated at the middle section. The preceding treatment shows that in the theory of shells it is quite unnecessary to introduce two-dimensional strains at all $\left({ }^{21}\right)$.

The two systems (3) and (4) are separate, so that (3) may be solved alone. As a consequence we see that if the load $(X, Y, Z)$ is independent of the thickness of the shell, so are the membrane stresses, and in any case the membrane stresses are not affected by the material of the shell. By considering different types of shells of varying thickness but the same middle section, one may get quite different displacement distributions corresponding to the same state of membrane stress.

The integrals of the membrane equations (3) and (4) may be written down at once $\left({ }^{22}\right)$ :

$$
\begin{aligned}
N_{\theta} & =-y \tan \alpha Z \\
N_{\theta y} & =-\frac{1}{y^{2}}\left[A(\theta)+\int\left(X-\sec \alpha \frac{\partial Z}{\partial \theta}\right) y^{2} d y\right] \\
N_{y} & =-\frac{1}{y}\left[B(\theta)+\int\left(Z \tan \alpha+\frac{\csc \alpha}{y} \frac{\partial N_{\theta y}}{\partial \theta}+Y\right) y d y\right], \\
V & =C(\theta)+\int\left(N_{y}-\nu N_{\theta}\right) \frac{d y}{E \delta}, \\
U & =y\left[D(\theta)+\int\left(\frac{2 y(1+\nu)}{E \delta} N_{\theta y}-\csc \alpha \frac{\partial V}{\partial \theta}\right) \frac{d y}{y^{2}}\right] \\
W & =\frac{y \tan \alpha}{E \delta}\left(N_{\theta}-\nu N_{y}\right)-\sec \alpha \frac{\partial U}{\partial \theta}-V \tan \alpha .
\end{aligned}
$$

${ }^{(20)}$ Truesdell (1943), pp. 51-54.

(21) For a correct "Hooke's law" for stress and moment resultants, see (8.10).

(22) The first three of these results were apparently first given in series form by Dischinger (1928); see Flügge (1934), pp. 45-46. 
In these results $E$ and $\delta$ may depend on $y$ and $\theta$. The arbitrary functions $A$ and $B$ represent two boundary conditions on the stresses out of the original four of the bending theory; the other two have been absorbed by the vanishing of the moments. The arbitrary functions $C$ and $D$ represent a rigid translation along the axis and a rigid rotation about the axis respectively, and may be chosen in any convenient way.

7. Examples. While we have seen that a membrane state of stress is not likely to exist near the apex of a closed cone, solutions for closed cones are of interest far from the apex. Let us suppose $X=O\left(y^{-1}\right), Y=O\left(y^{-1}\right), Z=O\left(y^{-1}\right)$ as $y \rightarrow 0$; then a necessary and sufficient condition for the resultants given by (6.6) to remain finite at the apex is that $A(\theta)=0$ and $B(\theta)=0$. In other words, for a given load distribution there can exist at most one boundary problem for a closed cone admitting a membrane state of stress near the apex. For example, suppose the cone is subject to its own weight:

$$
X=0, \quad Y=p \cos \alpha, \quad Z=p \sin \alpha,
$$

$p$ being the surface density of the shell (note that although we do not need to specify whether the thickness $\delta$ is a constant or not in order to obtain the following equations (2), they represent the effect of the shell's own weight only in the case of constant thickness, since we have assumed $p$ is constant in order to obtain them); then the only boundary problem which might be satisfied by membrane stresses near the apex is that for which

$$
N_{\theta}=-p y \sin \alpha \tan \alpha, \quad N_{\theta y}=0, \quad N_{y}=-p y \sec \alpha / 2 .
$$

Since this state of stress is independent of $\theta$ and since the total force across the boundary $y=y_{0}$ is directed axially and is of magnitude $\left[-\left(p y_{0} / 2\right) \sec \alpha\right][\cos \alpha]\left[2 \pi y_{0} \sin \alpha\right]$, equal to the weight of the shell above $y=y_{0}$, this solution is for the problem of a cone uniformly supported. From (6.6) we may compute the displacements, when $\delta$ is constant:

$$
\begin{aligned}
V & =p\left(l^{2}-y^{2}\right) \sec \alpha /(4 E \delta), \\
U & =0, \\
W & =p\left[(1+2 \nu) y^{2}-l^{2}\right] \sec \alpha \tan \alpha /(4 E \delta),
\end{aligned}
$$

where we have evaluated the constants of integration by prescribing that $U=0, V=0$ at the lower edge $y=l$.

Although the results (2) and (3) are solutions of the membrane equations which remain finite at the apex, nevertheless a membrane state of stress does not exist near the apex, for if we put these displacements back into (5.9) we find that although $M_{y \theta}=0, M_{\theta y}=0, N_{\theta y}=0, N_{y \theta}=0, M_{\nu} \approx 0$, and $M_{\theta} \approx 0$, nevertheless $N_{\theta}$ and $N_{y}$ become infinite as $y \rightarrow 0$. Then while the stress resultants (2) describe a membrane state of stress far from the apex, near the apex they are not justified at all, and are in fact meaningless. A closed cone stand- 
ing under its own weight does not admit a membrane state of stress near the apex, even though a solution of the membrane equations yielding formulas which remain finite at the apex exists for this problem.

A membrane state of stress may be inadmissible by the nature of the support at $y=l$. For example, there is no solution of the membrane theory equations finite at the apex and satisfying the condition $N_{\theta}=0$ at $y=l$, when the load is given by (1); we must resort to the bending theory in this case $\left({ }^{23}\right)$.

We may now ask, is it ever possible for a closed cone to admit a state of membrane stress near the apex? Since a necessary condition for finiteness at the apex is that $A(\theta)=0$ and $B(\theta)=0$, there must be axial symmetry; for simplicity let us suppose all quantities expansible in power series in $y$ near the apex, and let us suppose $\delta=O(1)$. Then the conditions that the terms following $K$ in the expressions (5.9) remain finite at $y=0$ may be shown to be

$$
V=V_{0}+\psi, \quad W=-V_{0} \tan \alpha+\phi,
$$

where $V_{0}$ is a constant and $\phi$ and $\psi$ are arbitrary power series starting with $y^{8}$. Putting these results into the first parts of the expressions (5.9) for $N_{\theta}$ and $N_{y}$, we see that these resultants may be arbitrary series starting with $y^{2}$, and hence by the equilibrium conditions (6.3) the loads $Y$ and $Z$ may be arbitrary series starting in $y$. For example, suppose

$$
X=0, \quad Y=p y \cos \alpha, \quad Z=p y \sin \alpha ;
$$

this load represents the weight of a uniform cone with varying density, or of a tapered cone of uniform density. The corresponding resultants and displacements are quickly found:

$$
\begin{aligned}
N_{\theta}= & -p y^{2} \sin \alpha \cos \alpha, \quad N_{\nu}=\left(-p y^{2} / 3\right) \sec \alpha, \\
V= & \frac{p\left(l^{3}-y^{3}\right)}{3 E \delta}(\sec \alpha / 3-\nu \sin \alpha \tan \alpha), \\
W= & \frac{p \tan \alpha}{E \delta}\left[y^{3}([1 / 3+\nu] \sec \alpha / 3-[1+\nu / 3] \sin \alpha \tan \alpha)\right. \\
& \left.-\frac{l^{3}}{3}(\sec \alpha / 3-\nu \sin \alpha \tan \alpha)\right] .
\end{aligned}
$$

These displacements are for a uniform and homogeneous cone, $\delta$ and $E$ both being constant; the solution (5), (6) is a correct first order approximation to a solution of the bending theory equations when $\delta /(y \tan \alpha)$ is small, valid even near the apex, and hence represents a membrane state of stress. For a tapered cone, say $\delta=k y$, the stresses (5) are still correct, but the displacements become, if $E$ is constant,

(23) For this example worked out in detail, see Flügge (1934), p. 165. 


$$
\begin{aligned}
V= & \frac{p\left(l^{2}-y^{2}\right)}{2 k E}(\sec \alpha / 3-\nu \sin \alpha \tan \alpha), \\
W= & \frac{p \tan \alpha}{k E}\left[y^{2}([\nu+1 / 2] \sec \alpha / 3-[1+\nu / 2] \sin \alpha \tan \alpha)\right. \\
& \left.-\frac{l^{2}}{2}(\sec \alpha / 3-\nu \sin \alpha \tan \alpha)\right] .
\end{aligned}
$$

The displacements (7), however, correspond to a membrane state of stress only far from the apex, so that the resultants (5) are now not correct in a region near the apex.

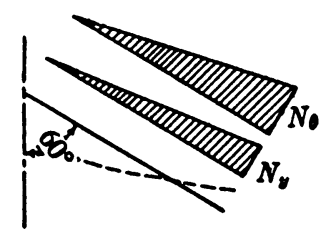

(a)

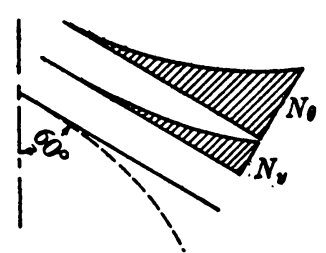

(b)

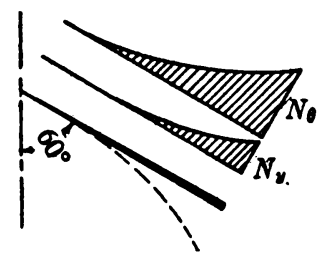

(c)
Stresses
Displacements
(a) Equations
(2)
Equations
(3)
(b) Equations
(5)
(c) Equations
(5)
Equations
Equations (7)

Stresses are read normal to the meridian. The broken line represents the deformed meridian, the displacements being greatly exaggerated.

Fig. 5.

The preceding three examples (see Fig. 5) are good instances of the flexibility of the membrane theory; for the load (1), a membrane state of stress can exist only far from the apex, while for the load (4), the same stress distribution (5) represents everywhere a membrane state of stress with displacements (6) if the thickness of the cone is constant, but is correct only far from the apex with displacements ( 7 ) if the cone is tapered. They indicate also the care with which the membrane theory should be handled.

Another problem of possible interest is that of a closed cone subject to wind pressure. Various authors have been accustomed to approximate wind pressure by a load of type:

$$
X=0, \quad Y=0, \quad Z=F \cos \alpha \cos \theta,
$$

supposing $F$ to be the surface force of the wind on a flat normal surface and the wind to be coming from the direction $\theta=0$, and experimental studies show that while such a load is not sufficient to fit wind pressure curves, it represents an important component (see (14.3)). The only stress distribution (6.6) finite at the apex is 


$$
\begin{gathered}
N_{\theta}=-F y \sin \alpha \cos \theta, \quad N_{y \theta}=(-F y / 3) \sin \theta, \\
N_{y}=(F y / 2) \cos \theta(\csc \alpha / 3-\sin \alpha) .
\end{gathered}
$$

It is interesting to notice that the shear is independent of the vertex angle $\alpha$, while for $\alpha=35^{\circ} 16^{\prime}$ the resultant $N_{y}$ vanishes everywhere. If we preclude a rigid body motion by prescribing $U=0, V=0$ for $y=l$, we obtain from (6.6) the displacements for a uniform cone:

$$
\begin{aligned}
& V=\frac{F\left(y^{2}-l^{2}\right) \cos \theta}{4 E \delta}[\csc \alpha / 3-(1-2 \nu) \sin \alpha] \\
& U=\frac{F \sin \theta}{E \delta}\left[-2(1+\nu)\left(l y-y^{2}\right) / 3+(y-l)^{2}\left(\csc ^{2} \alpha / 3-1+2 \nu\right) / 4\right] \\
& W=\frac{F \cos \theta \sec \alpha}{12 E \delta}\left[-y^{2}\left(9 \sin ^{2} \alpha+6+12 \nu+\csc ^{2} \alpha\right)\right. \\
& \left.\quad+2 y l\left(1+10 \nu+\csc ^{2} \alpha\right)+3 l^{2} \cot \alpha\left(\csc ^{2} \alpha / 3-(1-2 \nu)\right)\right] .
\end{aligned}
$$

A membrane state of stress exists only far from the apex.

Since little can be done in the membrane theory with a closed cone whose stresses are free of singularity, we shall now consider some examples when the stress is allowed to become infinite with $y^{-1}$ at the apex $y=0$. Such a singularity often occurs when the region of application of a load is diminished indefinitely; in this case the solution is said to represent a point load, and sufficiently far from the singularity it may be considered to approximate the stress and displacement patterns due to a load applied in a very small region near the point where the singularity occurs. First let us consider the effect of a load $P$ in the direction of the axis. For such a load the total force across any section of the cone, say $y=y_{0}$, should be axially directed and of magnitude $P$. From (6.6), since the cone is free of surface and body forces except at the apex, we obtain the stress resultants

$$
N_{y}=-P \csc 2 \alpha /(\pi y), \quad N_{\theta}=0, \quad N_{y \theta}=0 .
$$

We may check these results by comparing them with the solution of the threedimensional equations of elasticity given by Neuber( ${ }^{24}$ ) for an infinite tapered cone bearing an axial point load $P$ at the apex. In spherical coordinates $(r, \phi, \theta)$ this solution is

$$
\begin{aligned}
\tau_{r r} & =\frac{1}{r^{2}}[A-2(2+\nu) B \cos \phi+C], \\
\tau_{\phi \phi} & =\frac{1}{r^{2}}\left[-A \frac{\cos \phi}{1+\cos \phi}+(1-2 \nu) B \cos \phi+C \cot ^{2} \phi\right],
\end{aligned}
$$

(24) Neuber (1934), pp. 207-208. 


$$
\begin{aligned}
& \tau_{\theta \theta}=\frac{1}{r^{2}}\left[-A \frac{1}{1+\cos \phi}+(1-2 \nu) B \cos \phi-C \csc ^{2} \phi\right], \\
& \tau_{r \phi}=\frac{\sin \phi}{r^{2}}\left[-A \frac{1}{1+\cos \phi}+(1-2 \nu) B+C \cos \phi \csc ^{2} \phi\right], \\
& \tau_{r \theta}=0, \quad \tau_{\phi \theta}=0,
\end{aligned}
$$

where

$$
\begin{aligned}
& A=-\frac{1+\cos \beta \cos \gamma}{(1-\cos \beta)(1-\cos \gamma)} C, \\
& B=-\frac{C}{(1-2 \nu)(1-\cos \beta)(1-\cos \gamma)}, \\
& C=-\frac{P(1-2 \nu)(1-\cos \beta)(1-\cos \gamma)}{2 \pi(\cos \beta-\cos \gamma)\left(\cos ^{2} \beta+\cos ^{2} \gamma+2 \nu \cos \beta \cos \gamma\right)},
\end{aligned}
$$

the cone being bounded by $\phi=\gamma$ and $\phi=\beta, \gamma>\beta$. If in these results we make the substitutions $\phi \approx \alpha, \beta \approx \alpha, \gamma \approx \alpha, r(\gamma-\beta) \approx \delta, \tau_{r r}=N_{y} / \delta, \tau_{\theta \theta^{\circ}}=N_{\theta} / \delta$, $\tau_{\theta r}=N_{\theta y} / \delta$, we find that $\tau_{\phi \phi} \approx 0, \tau_{r \phi} \approx 0$, and we obtain the equations (11) for $N_{y}, N_{\theta}$, and $N_{\theta y}$. In this case then the solutions of the membrane equations give a correct first approximation to the solutions of the complete equations of the infinitesimal theory of elasticity when the cone is thin. The displacements corresponding to (11) for a cone of uniform thickness are

$$
\begin{aligned}
V & =-[P /(2 \pi E \delta)] \sec \alpha \csc \alpha \log (y / l), \\
U & =0, \\
W & =\left[P \sec ^{2} \alpha /(2 \pi E \delta)\right][\nu+\log (y / l)],
\end{aligned}
$$

where again we have prescribed that $U=0, V=0$ when $y=l$.

Another case of interest is that of pure torsion. The boundary conditions are that at $y=l, N_{y}=0$ and $N_{\theta y}=\left[M /\left(2 \pi l^{2}\right)\right] \csc ^{2} \alpha$, there being an axial moment $M$ applied at the vertex. We obtain a state of pure shear:

$$
\begin{array}{ll}
N_{\theta}=0, & N_{y}=0, \quad N_{\theta y}=M \cdot \csc ^{2} \alpha /\left(2 \pi y^{2}\right), \\
V=0, & W=0, \quad U=-\frac{M(1+\nu) \csc ^{2} \alpha}{2 \pi E \delta} \frac{l^{2}-y^{2}}{y},
\end{array}
$$

where the displacements are for a cone of uniform section.

Suppose now we have a uniform conical roof of surface density $p$ supported by a pillar at the apex (or held down by a guy wire at the apex) and supported at its base by several symmetrically placed equal pillars (Fig. 6). It is reasonable to suppose the structure so set up that conditions are the same at each pillar, and uniform across each, in other words, that at $y=l$ 


$$
N_{y}=\left\{\begin{array}{l}
0 \text { between the supports, } \\
P \text { (constant) at the supports. }
\end{array}\right.
$$

The most convenient way to find such a solution is to superpose upon the

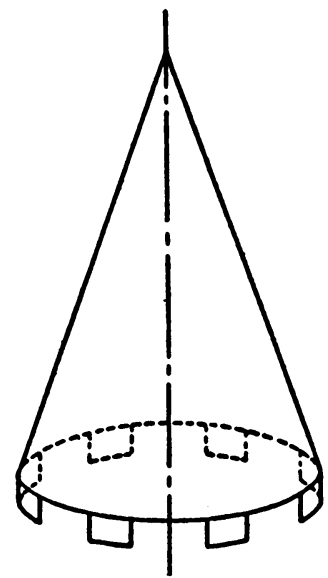

Fig. 6.

solution (2) a second solution for a load $X=0, Y=0, Z=0$, which satisfies at $y=l$ the boundary condition

$$
N_{y}=\left\{\begin{array}{l}
(p l / 2) \text { sec } \alpha \text { between the supports } \\
P^{\prime}(\text { constant) at the supports }
\end{array}\right\} \equiv f(\theta), \text { say. }
$$

We notice that, except at the discontinuities of $f, f^{\prime}(\theta)=0$. The solution contained in (6.6) satisfying the boundary condition (17) is

$$
\begin{aligned}
N_{0} & =0, \quad N_{\theta y}=0, \quad N_{y}=l f(\theta) / y, \\
V & =[f(\theta) /(E \delta)] \log (y / l), \quad U=0, \\
W & =-[f(\theta) /(E \delta)][\nu+\log (y / l)] \tan \alpha,
\end{aligned}
$$

where $U=0$ and $V=0$ at $y=l$, and $\delta$ is assumed constant. $P^{\prime}$ should be determined from consideration of the equilibrium of the whole structure. If we suppose the central column bears a load $H$, that the peripheral columns are $n$ in number and each of angular width $2 \epsilon$, then

$$
P^{\prime}=(p l / 2) \sec \alpha-\left(p \pi l^{2} \sin \alpha-H\right) /(2 \epsilon n l \sin \alpha \cos \alpha) .
$$

Fig. 7 shows graphs of the stress and displacement distribution for equations (2), (3), and (18) superposed, when $H=-T, T$ being a tension equal to twice the weight $p \pi l^{2} \sin \alpha$ of the cone, when $n=4, \epsilon=\pi / 32, \alpha=\pi / 3$. The lines drawn from the edges of the supports up to the vertex are lines of discontinuity of both stresses and displacements. 
When $H=0$, the column or guy wire may be removed and we may no longer speak of a point load, but the singularity at the apex does not disappear. Sufficiently far from the apex, the resulting solution will be the correct one for the cone with a free apex (see §16); the singularity in the membrane stresses does not necessarily indicate a singularity in the three-dimensional solution of the same problem, since when the stresses are $O(1)$ at $y=0$ the resultant $N_{y}$ may become infinite with $y^{-1}$, as can be seen from (4.1). The general character of the solution is as in Fig. 7, but the stresses and displacements are not so great.
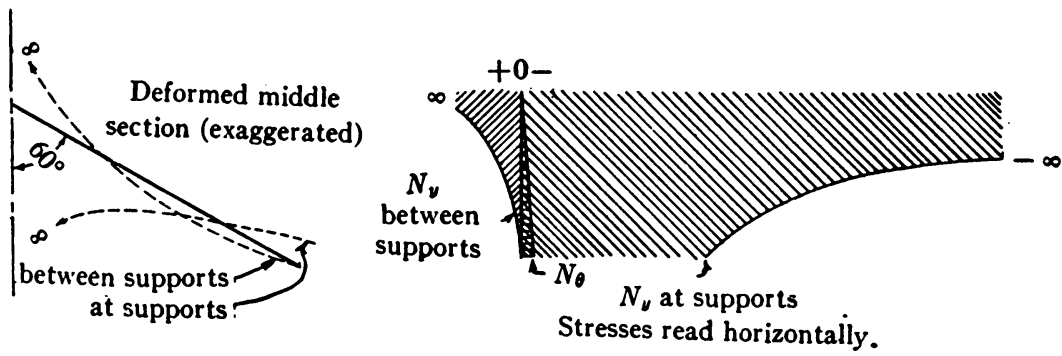

FIG. 7.

In this chapter we have seen from the example of the cone that the membrane theory produces ready solutions to fit a large variety of physical situations, but that while sometimes these solutions are correct approximations, sometimes they are not, depending on the region in which they are considered and the type of boundary solutions they are made to satisfy. We shall find results of the same character in the general theory of shells of revolution, which we now proceed to investigate.

\section{The DIFFERENTIAL EQUATIONS OF THE MEMbRANE THEORY FOR AN ARBITRARY SHELL OF REVOLUTION}

8. The differential equations of the bending theory. Using the expression (3.3) for the element of arc length, we can set up the equations of the threedimensional theory of elasticity in revolution coordinates. To put them in a convenient form we need to notice that

$$
\frac{\partial}{\partial \phi}\left[\left(r_{2}+x\right) \sin \phi\right]=\left(r_{1}+x\right) \cos \phi .
$$

To prove this relation, suppose that the Cartesian equation of the middle surface meridian in some axial plane is $r=f(z)$, where the $z$-axis is the axis of revolution. Then we have the geometrical formulas

(2) $f^{\prime}=-\cot \phi, \quad \frac{\partial}{\partial \phi}=\frac{\csc ^{2} \phi}{f^{\prime \prime}} \frac{\partial}{\partial z}, \quad r_{2}=f \csc \phi, \quad r_{1}=-\frac{\csc ^{3} \phi}{f^{\prime \prime}}$, 
from which (1) follows if we carry out the differentiation on the left. The equilibrium equations of three-dimensional elasticity $\left.{ }^{(25}\right)$ can now be put in the form

$$
\begin{aligned}
& \frac{\partial}{\partial \phi}\left[\left(r_{2}+x\right) \sin \phi \tau_{\phi \phi}\right]-\left(r_{1}+x\right) \cos \phi \tau_{\theta \theta}+\left(r_{1}+x\right) \frac{\partial \tau_{\theta \phi}}{\partial \theta} \\
& \quad+\frac{\sin \phi}{r_{1}+x} \frac{\partial}{\partial x}\left[\left(r_{1}+x\right)^{2}\left(r_{2}+x\right) \tau_{\phi x}\right]+\left(r_{1}+x\right)\left(r_{2}+x\right) \phi_{\phi} \sin \phi=0, \\
& \left(r_{1}+x\right) \frac{\partial \tau_{\theta \theta}}{\partial \theta}+\frac{\partial}{\partial \phi}\left[\left(r_{2}+x\right) \sin \phi \tau_{\theta \phi}\right]+\left(r_{1}+x\right) \cos \phi \tau_{\theta \phi} \\
& \quad+\frac{\sin \phi}{r_{2}+x} \frac{\partial}{\partial x}\left[\left(r_{1}+x\right)\left(r_{2}+x\right)^{2} \tau_{\theta x}\right]+\left(r_{1}+x\right)\left(r_{2}+x\right) \phi_{\theta} \sin \phi=0 \\
& \frac{\partial}{\partial x}\left[\left(r_{1}+x\right)\left(r_{2}+x\right) \tau_{x x}\right]-\left(r_{2}+x\right) \tau_{\phi \phi}-\left(r_{1}+x\right) \tau_{\theta \theta} \\
& \quad+\left(r_{1}+x\right) \csc \phi \frac{\partial \tau_{\theta x}}{\partial \theta}+\csc \phi \frac{\partial}{\partial \phi}\left[\left(r_{2}+x\right) \sin \phi \tau_{\phi x}\right]
\end{aligned}
$$

while the expressions $\left({ }^{26}\right)$ giving the strains in terms of the displacements become

$$
\begin{aligned}
\epsilon_{\phi \phi} & =\frac{1}{r_{1}+x}\left[\frac{\partial v}{\partial \phi}+w\right], \\
\epsilon_{\theta \theta} & =\frac{\csc \phi}{r_{2}+x}\left[\frac{\partial u}{\partial \theta}+v \cos \phi+w \sin \phi\right], \\
\epsilon_{x x} & =\frac{\partial w}{\partial x}
\end{aligned}
$$

$$
\begin{aligned}
& \epsilon_{\theta \phi}=\frac{\csc \phi}{2\left(r_{1}+x\right)\left(r_{2}+x\right)}\left[\left(r_{1}+x\right) \frac{\partial v}{\partial \theta}-u\left(r_{1}+x\right) \cos \phi\right. \\
& \left.+\left(r_{2}+x\right) \sin \phi \frac{\partial u}{\partial \phi}\right], \\
& \epsilon_{\theta x}=\frac{1}{2\left(r_{2}+x\right)}\left[\left(r_{2}+x\right) \frac{\partial u}{\partial x}-u+\csc \phi \frac{\partial w}{\partial \theta}\right], \\
& \epsilon_{\phi x}=\frac{1}{2\left(r_{1}+x\right)}\left[\left(r_{1}+x\right) \frac{\partial v}{\partial x}-v+\frac{\partial w}{\partial \phi}\right],
\end{aligned}
$$

(25) Love (1927), p. 90; Trefftz (1928), p. 81; Sokolnikoff (1941), p. 179.

(28) Love (1927), p. 54; Trefftz (1928), p. 78; Sokolnikoff (1941), pp. 177-178. 
where $u, v, w$ are the displacements in the directions of $\theta, \phi, x$ respectively. We shall now derive the equations of the bending theory as we did for conical shells in $\$ 5$. We shall not repeat the general discussion made at that time, for all our observations, with a few obvious changes, are effective here as well.

If we integrate equations (3), prescribing on $x=+\delta / 2$ and $x=-\delta / 2$ the boundary values $\tau_{x x}=-Z_{o}, \tau_{\phi x}=Y_{o}, \tau_{\theta x}=X_{0}$ and $\tau_{x x}=-Z_{i}, \tau_{\phi x}=Y_{i}, \tau_{\theta x}=X_{i}$ respectively, and introducing the quantities defined by equations (4.1) and (4.5), we obtain the equilibrium equations

$$
\frac{\partial}{\partial \phi}\left[r_{2} \sin \phi N_{\phi}\right]-r_{1} \cos \phi N_{\theta}+r_{1} \frac{\partial N_{\theta \phi}}{\partial \theta}-r_{2} \sin \phi Q_{\phi}+Y_{t} r_{1} r_{2} \sin \phi=0,
$$

$$
\begin{aligned}
& r_{1} \frac{\partial N_{\theta}}{\partial \theta}+\frac{\partial}{\partial \phi}\left[r_{2} \sin \phi N_{\phi \theta}\right]+r_{1} \cos \phi N_{\theta \phi}-r_{1} \sin \phi Q_{\theta}+X_{t} r_{1} r_{2} \sin \phi=0, \\
& \sin \phi\left(r_{2} N_{\phi}+r_{1} N_{\theta}\right)+\frac{\partial}{\partial \phi}\left[r_{2} \sin \phi Q_{\phi}\right]+r_{1} Q_{\theta}+Z_{t} r_{1} r_{2} \sin \phi=0 .
\end{aligned}
$$

If we multiply equations (3) by $x$ and then integrate, using also the definitions (5.6) we obtain two more equilibrium equations :

$$
\frac{\partial}{\partial \phi}\left[r_{2} \sin \phi M_{\phi}\right]-r_{1} \cos \phi M_{\theta}+r_{1} \frac{\partial M_{\theta \phi}}{\partial \theta}-r_{1} r_{2} \cdot \sin \phi\left(Q_{\phi}-L_{y}\right)=0,
$$

$$
r_{1} \frac{\partial M_{\theta}}{\partial \theta}+\frac{\partial}{\partial \phi}\left[r_{2} \sin \phi M_{\phi \theta}\right]+r_{1} \cos \phi M_{\theta \phi}-r_{1} r_{2} \sin \phi\left(Q_{\theta}-L_{x}\right)=0 .
$$

Again we suppose that the deformation will satisfy Assumptions (I) and (II) of $\$ 5$, expressed, of course, in revolution coordinates, and we deduce simplified Hooke's law equations similar to (5.5). The expressions for the displacements $u, v, w$ of the point $(\phi, \theta, x)$ in terms of the strains and of the displacements $U, V, W$ of its projection $(\phi, \theta, 0)$ on the middle surface are easily shown to be

(7)

$$
\begin{aligned}
u=U+ & \frac{x}{r_{2}}\left(U-\csc \phi \frac{\partial W}{\partial \theta}\right)+\int_{0}^{x} \frac{x-\xi}{r_{2}+\xi}\left(\frac{\partial \epsilon_{x x}}{\partial \theta} \csc \phi-2 \epsilon_{\theta x}\right) d \xi \\
& +2 \int_{0}^{x} \epsilon_{\theta x} d \xi, \\
v=V+ & \frac{x}{r_{1}}\left(V-\frac{\partial W}{\partial \phi}\right)+\int_{0}^{x} \frac{x-\xi}{r_{1}+\xi}\left(\frac{\partial \epsilon_{x x}}{\partial \phi}-2 \epsilon_{\phi x}\right) d \xi+2 \int_{0}^{x} \epsilon_{\phi x} d \xi, \\
w=W & +\int_{0}^{x} \epsilon_{x x} d \xi,
\end{aligned}
$$

which become, under Assumptions (I) and (II), simply 


$$
u \equiv U-x \frac{\frac{\partial W}{\partial \theta} \csc \phi-U}{r_{2}}
$$

$$
\begin{aligned}
v & \equiv V-x \frac{\frac{\partial W}{\partial \phi}-V}{r_{1}}, \\
w & =W,
\end{aligned}
$$

when terms of at most the second order in $x$ are retained. If we put these results into the expressions (4) for the strains, and with the aid of Hooke's law compute the integrals (4.1), we obtain a set of formulas, correct if we retain terms of at most fourth order in $\delta / R$, giving the resultants and moments in terms of $U, V, W, r_{1}, r_{2}$, and their derivatives; these expressions can be made symmetrical if we introduce the following abbreviations:

$$
\begin{aligned}
E_{\phi} & \left.\equiv \epsilon_{\phi \phi}\right|_{x=0},\left.\quad E_{\theta} \equiv \epsilon_{\theta \theta}\right|_{x=0},\left.\quad E_{\theta \phi} \equiv \epsilon_{\theta \phi}\right|_{x=0},\left.\quad E_{\phi \theta} \equiv \epsilon_{\phi \theta}\right|_{x=0}, \\
\kappa_{\theta} & =\frac{\csc \phi}{r_{2}}\left[\frac{1}{r_{2}}\left(\frac{\partial^{2} W}{\partial \theta^{2}} \csc \phi-\frac{\partial U}{\partial \theta}\right)+\frac{\cos \phi}{r_{1}}\left(\frac{\partial W}{\partial \phi}-V\right)\right], \\
\kappa_{\phi} & =\frac{1}{r_{1}} \frac{\partial}{\partial \phi}\left[\frac{1}{r_{1}}\left(\frac{\partial W}{\partial \phi}-V\right)\right],
\end{aligned}
$$

$$
\begin{aligned}
\mathfrak{E}_{\theta \phi} & \equiv E_{\theta \phi}-\frac{\csc \phi}{2 r_{1}}\left(\frac{\partial V}{\partial \theta}-U \cos \phi\right), \\
\mathfrak{E}_{\phi \theta} & \equiv E_{\phi \theta}-\frac{1}{2 r_{1}} \frac{\partial U}{\partial \phi}, \\
\kappa_{\theta \phi} & \equiv \frac{\csc \phi}{r_{2}}\left[\frac{1}{r_{1}}\left(\frac{\partial^{2} W}{\partial \theta \partial \phi}-\frac{\partial V}{\partial \theta}\right)+\frac{\cos \phi}{r_{2}}\left(\frac{\partial W}{\partial \theta} \csc \phi-U\right)\right], \\
\kappa_{\phi \theta} & \equiv \kappa_{\phi} / 2 .
\end{aligned}
$$

We may notice that since $E_{\theta \phi}=E_{\phi \theta}$, it follows that $\mathbb{E}_{\theta \phi}+\mathbb{E}_{\phi \theta}=E_{\theta \phi}$. With the aid of the abbreviations (9), the expressions for the resultants and moments become

$$
\begin{aligned}
& N_{\phi}=D\left[E_{\phi}+\nu E_{\theta}\right]+K\left(\frac{1}{r_{1}}-\frac{1}{r_{2}}\right)\left[\frac{1}{r_{1}} E_{\phi}+\kappa_{\phi}\right], \\
& N_{\theta}=D\left[E_{\theta}+\nu E_{\phi}\right]+K\left(\frac{1}{r_{2}}-\frac{1}{r_{1}}\right)\left[\frac{1}{r_{2}} E_{\theta}+\kappa_{\theta}\right], \\
& N_{\phi \theta}=D(1-\nu) E_{\phi \theta}+K(1-\nu)\left(\frac{1}{r_{1}}-\frac{1}{r_{2}}\right)\left[\frac{1}{r_{1}} E_{\theta \phi}+\kappa_{\phi \theta}\right],
\end{aligned}
$$




$$
\begin{aligned}
& N_{\theta \phi}=D(1-\nu) E_{\theta \phi}+K(1-\nu)\left(\frac{1}{r_{2}}-\frac{1}{r_{1}}\right)\left[\frac{1}{r_{2}} \xi_{\phi \theta}+\kappa_{\theta \phi}\right], \\
& M_{\phi}=K\left[\kappa_{\phi}+\nu \kappa_{\theta}+\left(\frac{1}{r_{1}}-\frac{1}{r_{2}}\right) E_{\phi}\right] \\
& M_{\theta}=K\left[\kappa_{\theta}+\nu \kappa_{\phi}+\left(\frac{1}{r_{2}}-\frac{1}{r_{1}}\right) E_{\theta}\right] \\
& M_{\theta \phi}=K(1-\nu)\left[\kappa_{\theta \phi}+\kappa_{\phi \theta}+\left(\frac{1}{r_{1}}-\frac{1}{r_{2}}\right) \xi_{\theta \phi}\right] \\
& M_{\phi \theta}=K(1-\nu)\left[\kappa_{\phi \theta}+\kappa_{\theta \phi}+\left(\frac{1}{r_{2}}-\frac{1}{r_{1}}\right) \xi_{\phi \theta}\right]
\end{aligned}
$$

where the moduli $D$ and $K$ are defined by (5.10). Equations (10) may be considered as a macroscopic Hooke's law, but we shall prefer to regard them merely as expressions giving the stress and moment resultants in terms of $U, V, W$ and their derivatives. We could write down similar expressions for $Q_{\phi}$ and $Q_{\theta}$, but we shall not need them.

The equations (5), (6), and (10) are the equations of the bending theory $\left({ }^{27}\right)$. A straightforward but prohibitive way to solve them would be to eliminate $Q_{\phi}$ and $Q_{\theta}$ from (5) by using (6), then to substitute the expressions (10) into the results, obtaining three partial differential equations in $U, V, W$ alone.

If the middle surface is a sphere, the formulas (10) are greatly simplified, and the shear resultants and moments become symmetrical. Practical solutions have been obtained for spheres even under unsymmetrical conditions by Schwerin ${ }^{(28)}$. If loads and boundary conditions are independent of $\theta$, then, whatever the form of the shell, the shear resultants and moments vanish, and a much simpler theory results, especially when, as is customary, in the equations (10) the terms following $K$ in the expressions for $N_{\phi}$ and $N_{\theta}$ and the terms involving $E_{\phi}$ and $E_{\theta}$ in the expressions for $M_{\phi}$ and $M_{\theta}$ are neglected; this simplified theory yields ready results for spheres and cones of constant

(27) The preceding derivation, deducing all the equations of the bending theory from threedimensional elasticity with the aid of Assumptions I and II, has not been given previously so far as I know. The first general treatment of thin shells is due to Aron (1874), who introduced averages rather than resultants. The first correct general treatment was given by Love in 1888; an account of his work may be found in Love (1927), chap. 24. A neat derivation in vector notation is given by $\mathrm{E}$. Reissner (1941): An elegant derivation in tensor notation of much more general equations of shell theory is given by Synge and Chien (1941), who develop separately a "macroscopic" and a "microscopic" theory; in the terminology of their paper, the present derivation deduces the macroscopic theory from the microscopic under the assumptions of small thickness and smaller strains and displacements. I have not followed the method of any of these authors.

(28) Schwerin (1918). 
thickness, and for tapered cones, and a discussion of it forms the bulk of the standard treatments of the bending theory $\left({ }^{29}\right)$. With these special theories we are not concerned here.

9. Singularities (bending theory). The expressions (8.10) give the terms up to the third order in the series expansions of the stress and moment resultants in powers of $\delta / R$, where $R \equiv \min \left(r_{1}, r_{2}\right)$, under the Assumptions (I) and (II) regarding the deformations. Under the assumptions of the three-dimensional theory of elasticity, that $U, V, W$, and their derivatives are all small, it is clear that when $|\delta / R|$ is sufficiently small the series will converge and the evaluations (8.10) will be sufficiently accurate. At a singularity of the middle surface of the type where $R \rightarrow 0$, the series will usually diverge; near a singularity, the expansion may or may not be justified, depending on the magnitude of $U, V, W$ and their derivatives. The divergence of one or more of these series does not mean that there is no three-dimensional solution of the problem in question, but that the description given by the bending theory is not a convenient one in this region.

For a surface free of singularity, if there exists a solution of the threedimensional equations satisfying prescribed values of the stresses on the boundaries $\left({ }^{30}\right)$, we may state at once an existence theorem for the bending theory: Under prescribed finite body forces and prescribed finite surface forces, for a thin shell with middle section an arbitrary given surface of revolution there exists a unique set of functions satisfying the bending theory partial differential equations such that the stress and moment resultants assume prescribed values, subject to the condition of equilibrium of the whole body, on the edges $\phi=\phi_{0}$ and $\phi=\phi_{1}$, provided that the strains given by the threedimensional theory satisfy the Assumptions (I) and (II).

The apex of a closed shell is of ten a singularity, for example, for a cone or for any pointed dome. Unfortunately it does not seem possible to make any general statements regarding the convergence of the series in problems concerning such shells. While the divergence of the series for some or all of the stress or moment resultants near the singularity may be of little concern to us if we are interested only in behavior far from the singularity in question, we need some boundary condition to be applied at such a point in order to obtain a solution valid elsewhere. It has been customary heretofore to require that all stress and moment resultants remain finite at the apex, and Flügge in a deceiving particular example $\left({ }^{31}\right)$ has claimed, wrongly, to show that this boundary condition is a requisite one. As a matter of fact, if finiteness at the apex were necessary to obtain a satisfactory solution, a great variety of prob-

(29) Flügge (1934), chap. 7; Timoshenko (1940), chap. 12.

${ }^{30}$ ) The existence of such a solution is probable from physical considerations. A mathematical existence theorem has been proved when the displacements, rather than the stresses, are prescribed on the boundaries; see Trefftz (1928), pp. 124-128.

(31) Flügge (1934), p. 40. 
lems of technical interest, in particular all those involving pointed shells under unsymmetrical loads or supports, would not be amenable to the treatment of the bending theory, as we shall see in $\$ 15$. Alternatively, if a small hole is cut out around the singularity, some of the resultants and moments may be made to vanish on the edge of this hole, and it might be argued that such a solution is the desired one, but it can be verified experimentally, say, for the cone, that the stress patterns given by such a solution depend significantly upon the dimensions of the hole, and that those resultants and moments which do not vanish become infinite as the size of the hole is diminished indefinitely. In any case either of these approaches is arbitrary, for near a singular point one might well expect the resultants to become infinite, as they clearly may (see the definitions (4.1)) even when the stress is quite small, without anything's being affected except the convergence of the series in $\delta / R$. When the series converge, term by term comparison indicates that the terms in $\delta$ must form an equilibrium system by themselves, as must the terms in $\delta^{3}$, and so on. As a boundary condition let us prescribe that the terms in $\delta$ must continue to form an equilibrium system in the limit at a singular point, even though the series may diverge and the terms become infinite, and similarly for the terms in $\delta^{3}$. Since the series usually diverge we may be going too far in prescribing this term by term equilibrium; I suggest it because it seems a reasonable boundary condition, automatically satisfied at all points which are not close to singularities, which will enable us to find well behaved solutions for numerous interesting problems not previously amenable to the methods of shell theory. When a solution of the equations of the bending theory satisfying in this sense the conditions of equilibrium cannot be found, we shall say simply that the problem in question is insoluble. Since we have preserved only two terms of the series in question, we shall never be able to show that a problem in question is not insoluble; we shall of ten, however, be able to say that a problem is insoluble from considering only the first or second terms, and if these two terms can be made to form equilibrium systems we shall describe the problem as soluble in the best sense which we can expect from the theory. In any case we shall not attempt to use these solutions near the singularity; we are imposing this boundary condition at a singularity simply to obtain solutions valid sufficiently far away from it.

We shall not pursue the study of singular points here, but we shall take up in detail in $\S \S 15-17$ their role in the membrane theory.

10. The equations of the membrane theory. Since except near a singularity $K / R^{2} \ll D$, the terms following $K$ in (8.10) may certainly be neglected in regions far from a singularity unless the boundary conditions prescribe that these terms are large. When it is justified to neglect these terms we describe the situation, as we did in the case of the cone, as a state of membrane stress. From equations (8.10) we see that the moments are negligible, that $N_{\theta \phi}=N_{\phi \theta}$, and that the expressions for $N_{\phi}, N_{\theta}$, and $N_{\theta \phi}$ are simplified. The 
equilibrium conditions (8.6) yield the results

$$
Q_{\phi}=L_{y}, \quad Q_{\theta}=L_{x} .
$$

If now we define effective load components $Z, Y, X$ :

$$
\begin{gathered}
Z=Z_{t}+\frac{1}{r_{1} r_{2} \sin \phi}\left[\frac{\partial}{\partial \phi}\left(r_{2} \sin \phi L_{y}\right)+r_{1} L_{x}\right], \\
Y=Y_{t}-L_{y} / r_{1} \\
X=X_{t}-L_{x} / r_{2}
\end{gathered}
$$

the remaining equilibrium equations become simply

$$
\frac{\partial}{\partial \phi}\left[r_{2} \sin \phi N_{\phi}\right]-r_{1} \cos \phi N_{\theta}+r_{1} \frac{\partial N_{\theta \phi}}{\partial \theta}+Y r_{1} r_{2} \sin \phi=0,
$$

$$
r_{1} \frac{\partial N_{0}}{\partial \theta}+\frac{\partial}{\partial \phi}\left[r_{2} \sin \phi N_{\phi \theta}\right]+r_{1} \cos \phi N_{\theta \phi}+X r_{1} r_{2} \sin \phi=0,
$$

$$
\frac{N_{\phi}}{r_{1}}+\frac{N_{\theta}}{r_{2}}=-Z
$$

while those of the equations (8.10) which do not vanish may be rearranged in the form

$$
\begin{aligned}
\frac{\partial V}{\partial \phi}+W & =\frac{r_{1}}{E \delta}\left(N_{\phi}-\nu N_{\theta}\right), \\
\frac{\partial U}{\partial \theta}+V \cos \phi+W \sin \phi & =\frac{r_{2} \sin \phi}{E \delta}\left(N_{\theta}-\nu N_{\phi}\right), \\
\frac{r_{2}}{r_{1}} \sin \phi \frac{\partial U}{\partial \phi}+\frac{\partial V}{\partial \theta}-U \cos \phi & =\frac{2(1+\nu) r_{2} \sin \phi}{E \delta} N_{\theta \phi} .
\end{aligned}
$$

These are the equations of the membrane theory. It is customary to treat problems in the bending theory by first solving the equations of the membrane theory under suitable boundary conditions and then superposing correcting terms involving solutions of the complete bending theory equations; that this process is justified is clear when we realize that the equations (3) and (4) are really the first terms in the expansions in powers of $\delta / R$ of the complete equilibrium and stress-displacement equations. When the shell is free of singularity and the boundary conditions are consistent with a membrane state of stress, a membrane state of stress will always exist for a thin enough shell. In practice it is observed that correcting terms supplied by the bending theory fall off exponentially from the supports, and that hence if a problem is soluble its solution, sufficiently far from singularities and supports, is given by the 
membrane theory. A solution of the systems (3) and (4) does not necessarily represent a state of membrane stress, however; near a singularity, when the series for the stress resultants diverge, the solutions may be meaningless, even though quite valid elsewhere (recall the examples given in \$7).

From the preceding remarks and the fact that the systems (3) and (4) are separate, the system (3) alone sufficing to determine the three stress resultants, it follows that sufficiently far from supports and singularities of a sufficiently thin shell, deformed in accordance with the assumptions of threedimensional elasticity and Assumptions (I) and (II), the state of stress is independent of the displacements and of the thickness and material of the shell, provided the loads $X, Y, Z$ are independent of the thickness of the shell. $X, Y$, and $Z$ may depend on $\delta$ through equations (2), (4.3), (4.4), and (4.6); if terms in $\delta / \mathrm{R}$ are neglected, then any load distribution $X, Y, Z$ is independent of $\delta$. Indeed, one state of stress leads to a variety of displacement distributions, depending on the thickness and material of the shell, which play an essential role in the equations (4). Its widespread adaptability, combined with the relative simplicity of its partial differential equations, makes the membrane theory at least the starting point in practically all elastic considerations of shells.

11. Stress functions. In order to solve the equations (10.3) it is convenient to introduce Fourier series:

$$
\begin{aligned}
& N_{\phi} \sim \sum_{-\infty}^{\infty} N_{\phi n} e^{i n \theta}, \quad N_{\theta} \sim \sum_{-\infty}^{\infty} N_{\theta n} e^{i n \theta}, \quad N_{\theta \phi} \sim \sum_{-\infty}^{\infty} N_{\theta \phi n} e^{i n \theta}, \\
& Z \sim \sum_{-\infty}^{\infty} Z_{n} e^{i n \theta}, \quad Y \sim \sum_{-\infty}^{\infty} Y_{n} e^{i n \theta}, \quad X \sim \sum_{-\infty}^{\infty} X_{n} e^{i n \theta} .
\end{aligned}
$$

The coefficients are functions of $\phi$ only. In practice cosine series for $N_{\phi}, N_{\theta}, Z$, and $Y$, and sine series for $N_{\theta \phi}$ and $X$, are usually sufficient; the coefficients of the cosine series will of course be $2\left(N_{\phi n}+N_{\phi,-n}\right)$, and so on. If we multiply equations $(10.3)$ by $(1 / 2 \pi) e^{-i n \theta} d \theta$ and integrate from 0 to $2 \pi$, we obtain the equations satisfied by the Fourier coefficients:

$$
\frac{N_{\phi n}}{r_{1}}+\frac{N_{\theta n}}{r_{2}}=-Z_{n}
$$

$\frac{d}{d \phi}\left(r_{2} \sin \phi N_{\phi n}\right)-r_{1} \cos \phi N_{\theta n}+i n r_{1} N_{\theta \phi n}+Y_{n} r_{1} r_{2} \sin \phi=0$,

$$
i n r_{1} N_{\theta n}+r_{1} \cos \phi N_{\theta \phi n}+\frac{d}{d \phi}\left(r_{2} \sin \phi N_{\theta \phi n}\right)+X_{n} r_{1} r_{2} \sin \phi=0 .
$$

These equations form a system of ordinary differential equations for the Fourier coefficients, but their complexity renders them unmanageable unless 
some tricks are used, or unless $n=0$. The analytically clumsy description of the middle surface in terms of its radii of curvature should be abandoned in favor of the Cartesian equation of the meridian introduced in \$8. Let us first define the stress functions of Nemenyi,

$$
\mathfrak{U}_{n} \equiv f r_{2} \sin ^{2} \phi N_{\phi n},
$$

and by putting (2) into (3) to eliminate $N_{\theta n}$ derive an expression for $N_{\theta \phi n}$ in terms of $\mathfrak{u}_{n}$ :

$$
i n r_{1} N_{\theta \phi n}=-\csc \phi \frac{d}{d \phi}\left(\frac{\mathfrak{u}_{n}}{f}\right)-Z_{n} r_{1} r_{2} \cos \phi-Y_{n} r_{1} r_{2} \sin \phi .
$$

Using (6) to eliminate $N_{\theta \phi n}$ from (4), and again using (2) and (5), we obtain an equaiıon satisfied by $\mathfrak{U}_{n}$ :

$$
-\frac{d}{d \phi}\left[\frac{r_{2}}{r_{1}} \frac{d}{d \phi}\left(\frac{\mathfrak{U}_{n}}{f}\right)\right]-\cot \phi \frac{d}{d \phi}\left(\frac{\mathfrak{U}_{n}}{f}\right)+n^{2} \csc ^{2} \phi \frac{\mathfrak{U}_{n}}{f}
$$

$$
\begin{aligned}
= & \frac{d}{d \phi}\left[Y_{n} r_{2}^{2} \sin ^{2} \phi+Z_{n} r_{2}^{2} \sin \phi \cos \phi\right] \\
& -Z_{n} r_{1} r_{2}\left(n^{2}-\cos ^{2} \phi\right)+Y_{n} r_{1} r_{2} \sin \phi \cos \phi-i n X_{n} r_{1} r_{2} \sin \phi .
\end{aligned}
$$

If we use the geometrical relations (8.2), this equation becomes simply

$$
\frac{d^{2} \mathfrak{U}_{n}}{d z^{2}}+\left(n^{2}-1\right) \frac{f^{\prime \prime}}{f} \mathfrak{U}_{n}=g_{n}(z),
$$

where

$$
\begin{aligned}
g_{n}(z) \equiv-\frac{d}{d z} & {\left[f^{2} f^{\prime} Z_{n}\right]+f\left[1+\left(n^{2}-1\right)\left(1+f^{\prime 2}\right)\right] Z_{n} } \\
& +f^{2} \frac{d Y_{n}}{d z}+3 f f^{\prime} Y_{n}+i n X_{n} f\left(1+f^{\prime 2}\right)^{1 / 2} .
\end{aligned}
$$

The equation (8) is the central equation of the membrane theory of shells of revolution.

The general solution of the equation (8) may be written in the form

$$
\mathfrak{u}_{n}=C_{n} \mathfrak{U}_{n 1}+D_{n} \mathfrak{U}_{n 2}+\mathfrak{u}_{n p},
$$

where $\mathfrak{U}_{n 1}$ and $\mathfrak{U}_{n 2}$ are two linearly independent solutions of the homogeneous equation associated with (8), $C_{n}$ and $D_{n}$ are arbitrary constants, and $\mathfrak{u}_{n p}$ is a particular integral. A particular integral may be written explicitly:

$$
\mathfrak{u}_{n p}=\frac{1}{\mathfrak{W}} \int_{a}^{s} g_{n}(\xi)\left[\mathfrak{u}_{n 2}(z) \mathfrak{U}_{n 1}(\xi)-\mathfrak{U}_{n 2}(\xi) \mathfrak{U}_{n 1}(z)\right] d \xi,
$$


where $a$ is any convenient point and the constant $\mathfrak{B}$ is the Wronskian determinant of $\mathfrak{u}_{n 1}$ and $\mathfrak{U}_{n 2}$.

Later we shall wish a notation for the complete solution:

$$
\mathfrak{U}_{n c} \equiv C_{n} \mathfrak{u}_{n 1}+D_{n} \mathfrak{u}_{n 2} \text {. }
$$

The $\mathfrak{U}_{n c}$ we shall call the complete stress functions, the $\mathfrak{U}_{n p}$ the particular stress functions.

When the stress functions $\mathfrak{U}_{n}$ have been chosen as solutions of (8), the system (2), (3), (4) will be satisfied if we obtain $N_{\phi n}, N_{\theta n}, N_{\theta \phi n}$ from

$$
\begin{gathered}
N_{\phi n}=\left(1+f^{\prime 2}\right)^{1 / 2} \mathfrak{U}_{n} / f^{2}, \quad N_{\theta n}=f^{\prime \prime} \mathfrak{U}_{n} /\left[f\left(1+f^{\prime 2}\right)^{1 / 2}\right]-f\left(1+f^{\prime 2}\right)^{1 / 2} Z_{n}, \\
i n N_{\theta \phi n}=\frac{d}{d z}\left(\frac{\mathfrak{U}_{n}}{f}\right)+f\left(f^{\prime} Z_{n}-Y_{n}\right) .
\end{gathered}
$$

The equation (8) is very easy to handle, as we shall see by numerous examples in Chapter IV.

The form of the equation (8) indicates that the stress functions will be real, since if $Z_{n}=\bar{Z}_{-n}, Y_{n}=\bar{Y}_{-n}$, and $X_{n}=\bar{X}_{-n}$, that is, if the loads are real, then the equation for $\mathfrak{U}_{n}$ is the same as that for $\overline{\mathfrak{u}}_{-n}$, so that by a proper arrangement of the constants $\mathfrak{U}_{n}=\overline{\mathfrak{U}}_{-n}$.

The stress functions (5) are very similar to those introduced by Nemenyi (1936) from graphical considerations; essentially the preceding derivation was given by Nemenyi and Truesdell (1943). A stress function for the membrane theory when the shell has a quite arbitrary middle surface has been given by Pucher (1938); it does not seem closely connected with these stress functions, and the differential equation for its Fourier coefficients for the case of a surface of revolution is more complicated than the equation (8). Pucher gave as examples of the use of his stress function a general solution for the spheroid in terms of hypergeometric functions and a simple algebraic solution for generalized paraboloids; these, and the original solutions of $\mathrm{H}$. Reissner (1912) for the sphere and Dischinger (1928) for the cone, were, so far as I know, the only general solutions of the system (11.2), (11.3), (11.4) published until the paper by Nemenyi and me (1943).

The relative usefulness and convenience of Pucher's and Nemenyi's stress functions may be seen by comparing Pucher's solution for spheroids, involving hypergeometric functions, with my simple algebraic solution (22.7) for a triply infinite family of surfaces including not only spheroids but also hyperboloids, ovoids, and more complicated surfaces.

12. The case of axial symmetry. In the case of axial symmetry, when $n=0$, the integral of (11.8) can easily be written explicitly. When $n=0$ in the third of equations (11.13) we may integrate directly, obtaining the result

$$
\mathfrak{u}_{0}=-f\left[\int_{a}^{z} f\left(f^{\prime} Z_{0}-Y_{0}\right) d \xi+C_{0}\right],
$$


where $C_{0}$ is an arbitrary constant and $a$ is any convenient point. From (1) and (11.13) we may compute $N_{\phi 0}$ and $N_{\theta 0}$; to find the shear $N_{\theta \phi 0}$, we may take the equation (11.4) when $n=0$ and after a little manipulation obtain the result

$$
N_{\theta \phi 0}=\frac{1}{f^{2}}\left[\int_{a}^{z} f^{2}\left(1+f^{\prime 2}\right)^{1 / 2} X_{0} d \xi+D_{0}\right],
$$

where $D_{0}$ is an arbitrary constant.

A loading of practical interest in the case when $n=0$ is that due to the surface density $p$ (not necessarily constant) of the shell: $Z_{0}=p \cos \phi$, $Y_{0}=p \sin \phi, X_{0}=0$, or

$$
Z_{0}=-p f^{\prime}\left(1+f^{\prime 2}\right)^{-1 / 2}, \quad Y_{0}=p\left(1+f^{\prime 2}\right)^{-1 / 2} .
$$

The integral (1) then assumes the form

$$
\mathfrak{u}_{0}=-f\left[\int_{a}^{2} p f\left(1+f^{\prime 2}\right)^{1 / 2} d \xi+C_{0}\right],
$$

whose geometric significance is obvious; doubtless this result was familiar to builders long before the science of mechanics was established.

The integral (1) has served as the basis of many engineering computations, some of which may be found in the standard treatments of the membrane theory $\left({ }^{32}\right)$; it is too well known to detain us here.

13. An example comparing the three-dimensional theory and the membrane theory. Suppose we have a sphere of radius $R$ and of constant thickness $\delta$ subject to a uniform internal pressure $p$. The appropriate solution of the three-dimensional equations is $\left({ }^{33}\right)$ :

$$
\begin{aligned}
\tau_{x x} & =\frac{p(R-\delta / 2)^{3}}{\delta\left(3 R^{2}+\delta^{2} / 4\right)}\left[1-\frac{(R+\delta / 2)^{3}}{(R+x)^{3}}\right], \\
\tau_{\theta \theta} & =\frac{p(R-\delta / 2)^{3}}{\delta\left(3 R^{2}+\delta^{2} / 4\right)}\left[1+\frac{(R+\delta / 2)^{3}}{2(R+x)^{3}}\right], \\
\tau_{\phi \phi} & =\tau_{\theta \theta}, \quad \tau_{x \theta}=0, \quad \tau_{x \phi}=0, \quad \tau_{\theta \phi}=0, \quad \epsilon_{x \theta}=0, \quad \epsilon_{x \phi}=0, \quad \epsilon_{\theta \phi}=0, \\
\epsilon_{x x} & =\frac{p(R-\delta / 2)^{3}}{\delta E\left(3 R^{2}+\delta^{2} / 4\right)}\left[1-2 \nu-(1+\nu) \frac{(R+\delta / 2)^{3}}{(R+x)^{3}}\right], \\
\epsilon_{\theta \theta} & =\frac{p(R-\delta / 2)^{3}}{\delta E\left(3 R^{2}+\delta^{2} / 4\right)}\left[1-2 \nu+\frac{(1+\nu)}{2} \frac{(R+\delta / 2)^{3}}{(R+x)^{3}}\right],
\end{aligned}
$$

(32) Flügge (1934), pp. 25-37; Timoshenko (1940), pp. 356-372.

(33) Love (1927), p. 142. 


$$
\begin{aligned}
\epsilon_{\phi \phi} & =\epsilon_{\theta \theta}, \quad u=0, \quad v=0, \\
w & =\frac{p(R-\delta / 2)^{3}}{\delta E\left(3 R^{2}+\delta^{2} / 4\right)}\left[(R+x)(1-2 \nu)+\frac{(1+\nu)}{2} \frac{(R+\delta / 2)^{3}}{(R+x)^{2}}\right],
\end{aligned}
$$

where the boundary conditions satisfied are that on $x=+\delta / 2, \tau_{x x}=0, \tau_{x \theta}=0$, $\tau_{x \phi}=0$, while on $x=-\delta / 2, \tau_{x x}=-p, \tau_{x \theta}=0, \tau_{x \phi}=0$.

Now in order for this solution to be correct in the three-dimensional theory it is necessary that $\left|\epsilon_{x x}\right| \ll 1,\left|\epsilon_{\theta \theta}\right| \ll 1,\left|\epsilon_{\phi \phi}\right| \ll 1$; then from (1), we see that it is necessary that $|p \nu / E| \ll \delta / R$. This condition is not so strong as Assumption (I), §5, which demands that $\left|\epsilon_{x x}\right| \ll \delta / R$, hence that

$$
|p \nu / E| \ll(\delta / R)^{2} \text {. }
$$

Assumption (II), §5, demanding that $\left|\tau_{x x} / E\right| \ll\left|\epsilon_{x x}+\nu \Delta /(1-\nu)\right|$ is always correct when $|\delta / R| \ll 1$. Hence (2) is the only condition which need be satisfied by the material and dimensions of the shell, provided only that the shell be thin, in order for the equations of the bending theory to be correct.

The range of applicability of our theory may be seen by an example. Suppose by " $a \ll 1$ " we agree to mean " $a \leqq .01$." Then for a spherical steel tank, for which $E=30,000,000 \mathrm{lbs}$./sq.in., $\nu=.3, \delta=1^{\prime \prime}, R=10^{\prime}$, we can treat pressures up to $65 \mathrm{lbs}$./sq.in. in the bending theory, and hence in the membrane theory, with accuracy. The three-dimensional theory, however, is accurate for pressures up to $7,000 \mathrm{lbs}$./sq.in.

It is sometimes assumed that the theory of shells is particularly applicable to very thin shells. This example, and indeed a moment's reflection would suffice, shows us that it is not applicable when the shell is too thin, and that in fact there is a range of thickness too small for the theory of shells and yet suitable to the three-dimensional theory. The explanation is, of course, that if the shell is too thin, the deformations will be too pronounced to satisfy the special assumptions of shell theory.

When the material and dimensions of the shell are such that condition (2) is satisfied, we are justified in using the expansions (8.10) and in retaining only the terms involving $D$. We obtain the results

$$
\begin{aligned}
& N_{\phi}=p R / 2, \quad N_{\theta}=p R / 2, \quad N_{\theta \phi}=0, \\
& U=0, \quad V=0, \quad W=(1-\nu) R^{2} p /(2 E \delta),
\end{aligned}
$$

exactly the solution which may be obtained directly in the membrane theory with the aid of the integral (12.1).

14. The case when $n= \pm 1$. If in (11.8) and (11.9) we consider the case when $n=1$, we find by integration and a little rearrangement that

$$
\begin{array}{r}
\mathfrak{U}_{1}=C_{1} z+D_{1}+\int_{a}^{z}\left[(z-\xi)\left(f Z_{1}+f f^{\prime} Y_{1}+i f\left(1+f^{\prime 2}\right)^{1 / 2} X_{1}\right)\right. \\
\left.-f^{2} f^{\prime} Z_{1}+f^{2} Y_{1}\right] d \xi
\end{array}
$$


where all the functions in the integrand are of the argument $\xi$, and $C_{1}$ and $D_{1}$ are arbitrary constants. A similar result holds when $n=-1$.

The case when $n= \pm 1$ is met most frequently in practice with a loading of type

$$
X=0, \quad Y=0, \quad Z=F \sin \phi \cos \theta
$$

or

$$
Z=-F\left(1+f^{\prime 2}\right)^{-1 / 2} \cos \theta
$$

which is used to represent wind pressure. Careful small scale experiments of Nemenyi( $\left.{ }^{(34}\right)$ have produced curves of wind pressure which might well be fitted with sufficient exactness by a load

$$
Z=A+B \cos \phi+C \sin \phi \cos \theta+D \sin \phi \cos 2 \theta,
$$

in which a term of type (2) occurs.

15. Closed domes. In this section we shall use the terms insoluble and soluble as defined in $\S 9$. A necessary condition for solubility is that the membrane stresses alone shall form an equilibrium system in the limit at the singularity, or, as we shall say briefly, that the problem is soluble in the membrane theory. A problem which is not soluble in the membrane theory is not soluble in the bending theory. We must remember that solubility in the membrane theory does not at all imply that a state of membrane stress exists near the singularity; a soluble problem, again, is one for which we can find a solution valid far from the singularity which, even though it may become infinite, continues to form an equilibrium system in the limit at the singularity.

We shall investigate the type of singularity of greatest practical interest, namely the apex of a closed dome, and we shall derive conditions for solubility in the membrane theory. Let us consider the equilibrium of a ring cut off by $\phi$ and $\phi+\Delta \phi$ in the limit as $\Delta \phi \rightarrow 0$. The conditions of equilibrium of this ring under membrane stresses only are

$$
\begin{aligned}
0= & \int_{0}^{2 \pi}\left[\frac{\partial}{\partial \phi}\left(N_{\phi} r_{2} \sin \phi \cos \phi\right)\left\{\begin{array}{l}
\cos \theta \\
\sin \theta
\end{array}\right\}+\frac{\partial}{\partial \phi}\left(N_{\theta \phi} r_{2} \sin \phi\right)\left\{\begin{array}{l}
\sin \theta \\
\cos \theta
\end{array}\right\}\right. \\
& \left.+r_{1} r_{2} \sin \phi\left([-Y \cos \phi+Z \sin \phi]\left\{\begin{array}{l}
\cos \theta \\
\sin \theta
\end{array}\right\}+X\left\{\begin{array}{l}
\sin \theta \\
\cos \theta
\end{array}\right\}\right)\right] d \theta \\
0= & \int_{0}^{2 \pi}\left[\frac{\partial}{\partial \phi}\left(N_{\phi} r_{2} \sin ^{2} \phi\right)-r_{1} r_{2} \sin \phi(Y \sin \phi+Z \cos \phi)\right] d \theta
\end{aligned}
$$

These conditions are satisfied in virtue of the differential equations (10.3) when the integrands are finite; but at an apex, where $r_{2} \sin \phi \rightarrow 0$, these integrands may not remain finite. Let us suppose the quantities $N_{\phi}, N_{\theta \phi}, X, Y$,

(34) Nemenyi (1936). 
and $Z$ replaced by their Fourier series. Then our conditions of equilibrium are that the quantities

$$
X_{n} r_{1} r_{2} \sin \phi, \quad Y_{n} r_{1} r_{2} \sin \phi \cos \phi, \quad Y_{n} r_{1} r_{2} \sin ^{2} \phi,
$$

$$
Z_{n} r_{1} r_{2} \sin \phi \cos \phi, \quad Z_{n} r_{1} r_{2} \sin ^{2} \phi,
$$

$$
\frac{d}{d \phi}\left[N_{\phi n} r_{2} \sin \phi \cos \phi\right], \quad \frac{d}{d \phi}\left[N_{\theta \phi n} r_{2} \sin \phi\right], \quad \frac{d}{d \phi}\left[N_{\phi n} r_{2} \sin ^{2} \phi\right]
$$

must all remain finite as $r_{2} \sin \phi \rightarrow 0$. With the aid of (8.2) and (11.13) the quantities (2) may be written in terms of the stress functions: the quantities

$$
\begin{aligned}
& \frac{1+f^{\prime 2}}{f^{\prime \prime}} \frac{d}{d z}\left[\frac{f^{\prime} \mathfrak{U}_{n}}{f}\right], \quad \frac{1+f^{\prime 2}}{f^{\prime \prime}} \frac{d}{d z}\left[\frac{\mathfrak{U}_{n}}{f}\right], \\
& \frac{1+f^{\prime 2}}{f^{\prime \prime}} \frac{d}{d z}\left[f \frac{d}{d z}\left(\frac{\mathfrak{U}_{n}}{f}\right)-f^{2}\left(f^{\prime} Z_{n}-Y_{n}\right)\right]
\end{aligned}
$$

must all remain finite as $f \rightarrow 0$.

We may suppose without loss of generality that the apex is at $z=0$. Near zero let the function $f$ be written in the form

$$
f=z^{\mu} \psi(z),
$$

where $\mu$ is a positive constant and $\psi$ is a power series such that $\psi(0) \neq 0$, a form which will suffice for cases of practical interest. When $\mu \neq 1 / 2$, the apex is a singularity of the shell; for any positive $\mu$ it may be a singular point of the differential equation (11.8) of the stress functions. When $\mu<1 / 2$, the apex is like a plate, with vanishing Gaussian curvature, and the element of arc length (3.3) and consequently the differential equations (10.3) are meaningless. In this case the boundary conditions (1) and (3) are clearly necessary conditions of equilibrium, since the series for the resultants and moments converge.

When $f$ is of the form (4), if there is a singularity of the differential equation (11.8) at $z=0$ it is regular( ${ }^{(35)}$, and we may easily calculate the exponents $\alpha_{n 1}$ and $\alpha_{n 2}$ of $\mathfrak{U}_{n 1}$ and $\mathfrak{U}_{n 2}\left({ }^{36}\right)$. We find that

$$
\begin{aligned}
& \alpha_{n 1}=\left(1+\left[1-4 \mu(\mu-1)\left(n^{2}-1\right)\right]^{1 / 2}\right) / 2, \\
& \alpha_{n 2}=\left(1-\left[1-4 \mu(\mu-1)\left(n^{2}-1\right)\right]^{1 / 2}\right) / 2 .
\end{aligned}
$$

When the apex is an ordinary point, the exponents are of course 0 and 1 .

We shall now work out conditions equivalent to (1) and (3) but expressed in terms of the loads and the exponent in (4). When a sum of terms must behave in a certain manner in the limit, we shall suppose each summand must

(35) The apex may not be a singularity of the differential equation at all, for example, if $f=z+z^{3}$ or $f=\sin z$.

(36) Those results in the theory of power series solutions of second order linear differential equations used in this paper may be found in Whittaker and Watson (1927), pp. 194-201. 
behave at worst in the same manner; then our resulting conditions will be sufficient but not quite necessary, since it is possible (though unlikely) that the sums which occur might have a cancelling effect. Strictly necessary conditions might be worked out in the same way, but they are too complicated to be useful. We shall consider $\mathfrak{U}_{n}$ written in the form (11.10). It is convenient to distinguish three types of shells :

Case I: $\mu<1$ (flat top),

Case II : $\mu=1$ (pointed top),

Case III : $\mu>1$ (spired top).

First let us consider the complete stress functions $\mathfrak{U}_{n c}$ only, for the case when $|n| \geqq 2$.

Case I. $\mu<1$. An easy calculation with the exponents (5) shows that $\mathfrak{U}_{n 2}$ will never satisfy conditions $(3)$, but that $\mathfrak{U}_{n 1}$ will satisfy them if

$$
\left[1-4 \mu(\mu-1)\left(n^{2}-1\right)\right]^{1 / 2} \geqq 3-2 \mu,
$$

or, equivalently, when

$$
|n| \geqq(2 / \mu)^{1 / 2} \text {. }
$$

When the condition (6) is satisfied, then the boundary condition at the apex is

$$
D_{n}=0 \text { when }|n| \geqq 2 .
$$

Case IIA. $\mu=1, f^{\prime \prime}(0) \neq 0$. The exponents are 0 and $1 ; \mathfrak{u}_{n 2}$ (which may be logarithmic) will not satisfy conditions $(3)$, but $\mathfrak{U}_{n 1}$, a power series starting in $z$, will satisfy them. The boundary condition is $(7)$.

Case IIB. $\mu=1, f^{\prime \prime}(0)=O\left(z^{a-2}\right)$, where $a$ is an integer greater than 2. Then $z=0$ is an ordinary point of the differential equation. $\mathfrak{U}_{n 2}$, a power series with a constant term, is again unsatisfactory. It is possible to show that $\mathfrak{U}_{n 1}=z+A z^{a}+\cdots$, and hence conditions (3) will be satisfied by it. The boundary condition again is (7).

(These two possibilities exhaust Case II for a shell of form (4).)

Case III. $\mu>1$. When $|n| \geqq 2$ the exponents (5) are conjugate complex numbers with real part equal to $1 / 2$. The complete stress functions may be written in the real form

$$
\mathfrak{u}_{n c}=z^{1 / 2}\left[A_{n} \cos \left(\beta_{n} \log z\right) \phi_{1 n}+B_{n} \sin \left(\beta_{n} \log z\right) \phi_{2 n}\right],
$$

where $\beta_{n}$ is the imaginary part of the exponents and $\phi_{1 n}$ and $\phi_{2 n}$ are power series which do not vanish at $z=0$. The conditions (3) can never be satisfied when $|n| \geqq 2$.

The cases when $n=0$ and $n= \pm 1$ are of no interest when there is no load.

Let us call the part of $N_{\phi n}$ dependent on $\mathfrak{U}_{n 1}$, say, $N_{\phi n}^{(1)}$. It is interesting to determine the behavior of $N_{\phi n}^{(1)}$ at the apex, when $|n| \geqq 2$. This behavior may be observed from the formulas (11.12) and the exponents (5).

Case I. $N_{\phi n}^{(1)}$ is zero, finite, or infinite according as $\alpha_{n 1}>\mu+1, \alpha_{n 1}=\mu+1$, 
$\alpha_{n 1}<\mu+1$; in other words, as we can easily verify, according as

(8) $|n|>[2 /(1-\mu)]^{1 / 2},|n|=[2 /(1-\mu)]^{1 / 2}$, or $|n|<[2 /(1-\mu)]^{1 / 2}$.

Cases II and III. In this case $N_{\phi n}^{(1)}$ is finite, zero, or infinite according as $R l \alpha_{n 1}>2 \mu, R l \alpha_{n 1}=2 \mu$, or $R l \alpha_{n 1}<2 \mu$. Hence we may easily see it is always infinite.

With the aid of (6) and (8) we may now construct a table summarizing these results. In each entry, besides the words "soluble" or "insoluble," we have put the value approached at the apex by $N_{\phi n}^{(1)}$.

\begin{tabular}{|c|c|c|c|c|c|}
\hline & $|n|=2$ & $|n|=3$ & $|n|=4$ & $|n|=5$ & $\cdots$ \\
\hline & $\dot{\bullet} \cdot$ & $\dot{.}$ & $\dot{.}$ & • & $\dot{.}$ \\
\hline $2 / 16>_{\mu} \geqq 2 / 25$ & $\begin{array}{c}\text { insoluble } \\
\text { zero }\end{array}$ & $\begin{array}{c}\text { insoluble } \\
\text { zero }\end{array}$ & $\begin{array}{c}\text { insoluble } \\
\text { zero }\end{array}$ & $\begin{array}{c}\text { soluble } \\
\text { zero }\end{array}$ & $\cdots$ \\
\hline $2 / 9>_{\mu} \geqq 2 / 16$ & $\begin{array}{c}\text { insoluble } \\
\text { zero }\end{array}$ & $\begin{array}{c}\text { insoluble } \\
\text { zero }\end{array}$ & $\begin{array}{c}\text { soluble } \\
\text { zero }\end{array}$ & $\begin{array}{c}\text { soluble } \\
\text { zero }\end{array}$ & $\cdots$ \\
\hline $2 / 4>\mu \geqq 2 / 9$ & $\begin{array}{c}\text { insoluble } \\
\text { zero }\end{array}$ & $\begin{array}{c}\text { soluble } \\
\text { zero }\end{array}$ & $\begin{array}{c}\text { soluble } \\
\text { zero }\end{array}$ & $\begin{array}{c}\text { soluble } \\
\text { zero }\end{array}$ & $\cdots$ \\
\hline$\mu=1 / 2$ & $\begin{array}{l}\text { soluble } \\
\text { finite }\end{array}$ & $\begin{array}{c}\text { soluble } \\
\text { zero }\end{array}$ & $\begin{array}{c}\text { soluble } \\
\text { zero }\end{array}$ & $\begin{array}{c}\text { soluble } \\
\text { zero }\end{array}$ & $\cdots$ \\
\hline $2 / 4<\mu<7 / 9$ & $\begin{array}{l}\text { soluble } \\
\text { infinite }\end{array}$ & $\begin{array}{c}\text { soluble } \\
\text { zero }\end{array}$ & $\begin{array}{c}\text { soluble } \\
\text { zero }\end{array}$ & $\begin{array}{c}\text { soluble } \\
\text { zero }\end{array}$ & $\cdots$ \\
\hline$\mu=7 / 9$ & $\begin{array}{l}\text { soluble } \\
\text { infinite }\end{array}$ & $\begin{array}{l}\text { soluble } \\
\text { finite }\end{array}$ & $\begin{array}{c}\text { soluble } \\
\text { zero }\end{array}$ & $\begin{array}{c}\text { soluble } \\
\text { zero }\end{array}$ & $\cdots$ \\
\hline $7 / 9<\mu<14 / 16$ & $\begin{array}{l}\text { soluble } \\
\text { infinite }\end{array}$ & $\begin{array}{l}\text { soluble } \\
\text { infinite }\end{array}$ & $\begin{array}{c}\text { soluble } \\
\text { zero }\end{array}$ & $\begin{array}{c}\text { soluble } \\
\text { zero }\end{array}$ & $\cdots$ \\
\hline$\mu=14 / 16$ & $\begin{array}{l}\text { soluble } \\
\text { infinite }\end{array}$ & $\begin{array}{l}\text { soluble } \\
\text { infinite }\end{array}$ & $\begin{array}{l}\text { soluble } \\
\text { finite }\end{array}$ & $\begin{array}{c}\text { soluble } \\
\text { zero }\end{array}$ & \\
\hline - & - & - & - & - & • \\
\hline - & - & - & - & - & - \\
\hline - & - & - & - & - & $\cdot$ \\
\hline$\mu=1$ & $\begin{array}{l}\text { soluble } \\
\text { infinite }\end{array}$ & $\begin{array}{l}\text { soluble } \\
\text { infinite }\end{array}$ & $\begin{array}{l}\text { soluble } \\
\text { infinite }\end{array}$ & $\begin{array}{l}\text { soluble } \\
\text { infinite }\end{array}$ & $\cdots$ \\
\hline$\mu>1$ & $\begin{array}{l}\text { insoluble } \\
\text { infinite }\end{array}$ & $\begin{array}{l}\text { insoluble } \\
\text { infinite }\end{array}$ & $\begin{array}{l}\text { insoluble } \\
\text { infinite }\end{array}$ & $\begin{array}{c}\text { insoluble } \\
\text { infinite }\end{array}$ & $\cdots$ \\
\hline
\end{tabular}


From table (9) we can see that the condition of finiteness at the apex which has been used up to the present as a boundary condition $\left({ }^{37}\right)$ cannot be satisfied by a large class of surfaces, in particular by any pointed dome, when $|n| \geqq 2$, but that the boundary condition used in this section can be satisfied by all surfaces of practical interest when $1 / 2 \leqq \mu \leqq 1$, and will lead to solutions which are correct sufficiently far from the apex.

Table (9) tells us also when it is possible for there to be a membrane state of stress at the apex. If the problem concerns all values of $n$ which are absolutely greater than 1 , then a necessary condition for a membrane state of stress at the apex is $\mu=1 / 2$, that is, that both radii of normal curvature are finite and not zero, and the apex is like that of a sphere. To see whether a membrane state of stress actually does exist there, we must calculate the displacements and estimate the terms neglected in (8.10) (cf. the examples in $\S 7$ ).

Now we must consider the effect of the load. Suppose that at the apex

$$
Z_{n}=O\left(z^{\rho(n)}\right), \quad Y_{n}=O\left(z^{v(n)}\right), \quad X_{n}=O\left(z^{\chi(n)}\right) .
$$

Then conditions (1) may be expressed in terms of these exponents.

(11) Case I: $\rho(n) \geqq 2(1-2 \mu), v(n) \geqq 2(1-2 \mu), \chi(n) \geqq 1-3 \mu$. Case II : If $f^{\prime \prime}(z)=O\left(z^{a}\right)$, where $a$ is zero or a positive integer, then $\rho(n) \geqq a-1, v(n)$ $\geqq a-1, \chi(n) \geqq a-1$.

In view of our preceding discussion, Case III is not of interest, except possibly when $n=0$ or $n= \pm 1$. One result contained in the criterion (11) is that if $\mu=1 / 2$, any loading finite or zero at the apex will lead to a soluble problem; another is that if $\mu=1$ and $f^{\prime \prime}(z)=A+B z+\cdots, A B \neq 0$ (and this is the case of greatest interest), any such loading will again lead to soluble problems.

We must also consider the effect of the loads on the particular integral $\mathfrak{U}_{n p}$. Suppose that at the apex $g_{n}(z)=O\left(z^{\gamma(n)}\right)$; then an easy computation in (11.11) with the exponents (5) shows that $\mathfrak{U}_{n p}=O\left(z^{\gamma(n)+2}\right)$, provided no logarithmic terms arise due to the integration. This result is not altered even if $\mathfrak{U}_{n 2}$ is logarithmic, for the logarithmic terms due to it in $\mathfrak{u}_{n p}$ will cancel. Using this estimate of $\mathfrak{U}_{n p}$ and the form of $g_{n}(z)$ as given by (11.9), we can show that the conditions (11) are sufficient to assure that $\mathfrak{U}_{n p}$ will satisfy the conditions (3). Under the same conditions (11), the terms in (3) in which $Y_{n}$ and $Z_{n}$ occur explicitly will not occasion trouble. If a logarithmic term appears in $\mathfrak{u}_{n p}$ due to the integration, as it can if there is a term in $g_{n}(z)$ involving $z^{-\alpha}$, where $\alpha$ equals either of the exponents (15.5) + a positive integer, then $\mathfrak{u}_{n p}=O\left(z^{\gamma(n)+2} \log z\right)$, and the signs " $\geqq$ " in (11) must be replaced by " $>$."

In a similar way we can calculate the limiting value of that part of $N_{\phi n}$ due to the dependence of $\mathfrak{U}_{n p}$ on $Z_{n}, Y_{n}, X_{n}$ respectively, at the apex, using

(37) Flügge (1934), pp. 40, 48, et passim. 
the formulas (11.12); in view of the insolubility of problems of Case III when $|n| \geqq 2$, we shall confine ourselves to the Cases I and II.

(12) The part due to $Z_{n}$ is zero if $\rho(n)>1-2 \mu$, finite but not zero if $\rho(n)=1-2 \mu$, infinite if $\rho(n)<1-2 \mu$. The part due to $Y_{n}$ is zero if $v(n)>-\mu$, finite but not zero if $v(n)=-\mu$, infinite if $v(n)<-\mu$. The part due to $X_{n}$, when $n \neq 0$, is zero if $\chi(n)>-\mu$, finite but not zero if $\chi(n)=-\mu$, infinite if $\chi(n)<-\mu$.

In case logarithmic terms occur in $\mathfrak{u}_{n p}$, the corresponding cases of finite but nonzero stress in (12) should be deleted and incorporated in the infinite case.

For completeness we should calculate similar results for the cases when $n=0$ and $n= \pm 1$. For these cases we use the integrals (12.1), (12.2), and (14.1). The criteria (11) are still valid. By a proper choice of $C_{0}$ and $D_{0}$, any problem of Cases I and II when $n=0$ is soluble if the conditions (11) are satisfied; the value of $N_{\phi 0}$ at the apex is given by (12). By a proper choice of $D_{1}$, a problem when $n= \pm 1$ is soluble in Cases I and II only, and if and only if the conditions (11) are satisfied; the stress $N_{\phi 1}$ or $N_{\phi,-1}$ will be infinite at the apex unless $C_{1}$ is properly chosen, in which case its value will be given by (12). The criteria for Case III when $n=0$ are as follows: the problem is soluble if $\rho(0) \geqq-2, v(0) \geqq-2, \chi(0) \geqq-2 ; N_{\phi 0}$ is zero at the apex if $\rho(0)>-1$ and $v(0)>-1$, finite but not zero if $\rho(0)=-1$ and $v(0)=-1$, or if $\rho(0)=-1$ and $v(0)>-1$, or if $\rho(0)>-1$ and $v(0)=-1$, and infinite if $\rho(0)<-1$ or if $v(0)<-1 ; N_{\theta \phi 0}$ is zero at the apex if $\chi(0)>-1$, finite but not zero if $\chi(0)=-1$, and infinite if $\chi(0)<-1$.

With the foregoing results one may determine immediately the solubility of a particular problem and the behavior of the stresses at the apex. For example, consider the load (12.3) due to the weight of the shell; here $\rho(0)=0$, $v(0)=1-\mu$ in Case I, $v(0)=0$ in Cases II and III. Then, from (11), we may see that in Case I, the problem of a shell under its own weight, uniformly supported, is soluble if $\mu \geqq 1 / 2$; in Case II it is soluble if $f^{\prime \prime}(z)=A+B z+\cdots$, $A B \neq 0$; in Case III it is always soluble. When $\mu=1 / 2, N_{\phi 0}$ is finite at the apex; if $\mu>1 / 2$, it is zero. If now instead of supporting this shell uniformly we support it on $m$ symmetrical columns, we shall need (see $\$ 21$ ) solutions involving $\mathfrak{u}_{n 1}$ when $n=m, 2 m, \cdots$, in addition to the previous solution. Then from (9), the problem will be soluble if $1 / 2 \leqq \mu \leqq 1$; from (8), the stress $N_{\phi}$ will be finite or infinite at the apex according as $m>[2 /(1-\mu)]^{1 / 2}$ or $m \leqq[2 /(1-\mu)]^{1 / 2}$, and in particular, it will always be infinite when $\mu=1$, finite when $\mu=1 / 2$.

The mere recitation of the results of this section has been lengthy and tedious. The patient reader, if he has persevered this far, will readily excuse my omitting practically all steps of the proofs, since they are lengthy but straightforward calculations, and he may in fact wonder why I have carried out these investigations at all. When I began to study the membrane theory I was particularly interested in Case II (pointed domes), and was surprised first to notice that in all the examples of unsymmetrical loads or supports 
which I could work out the stress $N_{\phi}$ became infinite at the apex, and second to observe that all the works on the membrane theory which I could find with one exception simply overlooked, probably because of the complications in calculation by all previous methods, any unsymmetrical problems involving pointed domes. The exception was Flügge's approximate numerical example which I shall discuss in $\$ 24$, but by exhibiting an exact solution for Flügge's problem I found that his result, as far as the apex was concerned, was incorrect. Was it then impossible to treat any pointed domes in the membrane theory? In considering this question I undertook the preceding investigation, which shows that the answer must be "yes" if the old boundary condition of finiteness at the apex is used. By formulating the new boundary conditions (1) and (3) I have been able to treat any pointed dome (Case II). The preceding investigation was necessary to show that the new boundary conditions can really be satisfied in a variety of problems where the old can not. The spired dome (Case III) remains excluded from the theory of shells, except in the case of axial symmetry of load and support. Both the new and the old boundary conditions can be satisfied always for a dome with a spherelike apex $(\mu=1 / 2)$ for any finite load system. The remainder of Case I, when $0<\mu<1 / 2$ and when $1 / 2<\mu<1$, is not of much technical interest. We may roughly summarize the results of technical interest in this section:

I. If the loading does not become infinite at the apex, a problem involving a dome with a sphere-like apex $(\mu=1 / 2)$ is soluble, the correct boundary condition at the apex being (7), and the stress resultants will be finite, possibly zero, at the apex.

II. Under the same conditions a problem involving a pointed dome $(\mu=1)$ is soluble if $f^{\prime \prime}(z)=A+B z+\cdots, A B \neq 0$, the correct boundary condition at the apex being again (7), but now the stress resultant $N_{\phi}$ will be infinite at the apex, except possibly in the case of axially symmetric loading and support.

Thus we see that the problems of greatest technical interest can all be treated satisfactorily in the membrane theory. That the stress functions afford an easy and efficient approach for numerical results as well as for the general considerations for which we have used them up to now, we shall see in Chapter IV. In particular, that the infinite $N_{\phi}$ at the apex of a pointed dome does not detract from the practical usefulness of the solution we shall see by an example in $\$ 24$.

16. A note on the cone. From all the preceding discussions one surface for which $\mu=1$ must be excluded, the cone, since the original coordinate system in which the partial differential equations (10.3) were expressed is not valid for it. By using the integrals (6.6) we may parallel the discussion (I spare the reader the details) and reach the following conclusions. If $X=O\left(y^{-1}\right)$, $Y=O\left(y^{-1}\right)$, and $Z=O\left(y^{-1}\right)$ at the apex, the conditions of equilibrium at the apex can be satisfied, and the boundary condition is

$$
A(\theta)=0 .
$$


We have already seen in $\$ 7$ that the stresses can be finite at the apex in cases of axially symmetric load and support only. These results show that the cone is no exception in Case II. In particular, the solution (7.18), (7.19) when $H=0$, for a cone supported on several pillars, while it seemed arbitrarily selected, may now be seen to be correct sufficiently far from the apex.

17. Open domes. If we have a shell free of singularity when $\phi_{0} \leqq \phi \leqq \phi_{1}$, it is clear that we may find a solution of the membrane equations satisfying one prescribed condition at $\phi=\phi_{0}$ and one at $\phi=\phi_{1}$.

While many practical problems concern domes free of singularity, some interesting open domes are singular at one boundary, and hence must be treated as in $\$ 15$. We shall consider one such type of dome, where

$$
f(z)=\phi(z)+z^{\nu} \psi(z), \quad 0<\nu<1,
$$

$\phi$ and $\psi$ being power series such that $\phi(0) \neq 0, \psi(0) \neq 0$. Such a dome has a flat top with a hole of radius $\phi(0)$ in it. The singularity of the differential equation (11.8) becomes regular if we choose $z^{\nu}$ as a new independent variable, and the exponents $\beta_{n 1}, \beta_{n 2}$ at the origin are

$$
\beta_{n 1}=1 / \nu, \quad \beta_{n 2}=0 .
$$

Hence $\mathfrak{U}_{n 1}=z+\cdots, \mathfrak{U}_{n 2}=1+\cdots$, but the criteria will not be the same as for $\$ 15$, Case II, since $f^{\prime \prime}(z)=A z^{\nu-2}+\cdots$. Conditions (15.3) are satisfied if and only if $\nu \geqq 1 / 2$, the boundary condition again being $D_{n}=0 . N_{\phi n}^{(1)}$ vanishes at $z=0$ in all cases. We shall not devote the space required for a detailed analysis like that of $\S 15$; an example of the difference in behavior of the stresses for two shells of parabolic meridian, one of type (15.4) and one of type (1), may be seen in $\$ 21$, Fig. 8, and $\$ 25$, Fig. 19.

18. Displacement functions. When the equilibrium equations $(10.3)$ have been solved by the method of $\S 11$, the quantities on the right-hand sides of equations (10.4) are known, at least up to two arbitrary functions, and we may regard the system (10.4) as three equations for $U, V, W$. Let us introduce Fourier series for these quantities:

$$
U \sim \sum_{-\infty}^{\infty} U_{n} e^{i n \theta}, \quad V \sim \sum_{-\infty}^{\infty} V_{n} e^{i n \theta}, \quad W \sim \sum_{-\infty}^{\infty} W_{n} e^{i n \theta},
$$

where $U_{n}, V_{n}$, and $W_{n}$ are functions of $\phi$ alone. If we multiply the equations (10.4) by $(1 / 2 \pi) e^{-i n \theta} d \theta$ and integrate from 0 to $2 \pi$, we obtain the equations of the Fourier coefficients :

$$
\begin{gathered}
\frac{d V_{n}}{d \phi}+W_{n}=\frac{r_{1}}{E \delta}\left(N_{\phi n}-\nu N_{\theta n}\right), \\
i n U_{n}+V_{n} \cos \phi+W_{n} \sin \phi=\frac{r_{2} \sin \phi}{E \delta}\left(N_{\theta n}-\nu N_{\phi n}\right),
\end{gathered}
$$




$$
\frac{r_{2}}{r_{1}} \sin \phi \frac{d U_{n}}{d \phi}-U_{n} \cos \phi-i n V_{n}=\frac{2 r_{2}(1+\nu) \sin \phi}{E \delta} N_{\theta \phi n} .
$$

These equations form a system of ordinary differential equations for the Fourier coefficients, but their complexity renders them unmanageable unless some tricks are used, or unless $n=0\left({ }^{38}\right)$. Let us introduce the displacement functions $\mathfrak{B}_{n}$ :

$$
\mathfrak{B}_{n} \equiv V_{n} \csc \phi .
$$

Eliminating $W_{n}$ from (2) and (3), and using (5), we find an expression for $U_{n}$ in terms of the displacement function $\mathfrak{B}_{n}$ :

$$
i n U_{n}=\sin ^{2} \phi \frac{d \mathfrak{B}_{n}}{d \phi}+\frac{r_{2} \sin \phi}{E \delta}\left(N_{\theta n}-\nu N_{\phi n}\right)-\frac{r_{1} \sin \phi}{E \delta}\left(N_{\phi n}-\nu N_{\theta n}\right) .
$$

If we put (5) and (6) into (4) we obtain a differential equation for $\mathfrak{B}_{n}$ :

$$
\begin{aligned}
\frac{r_{2}}{r_{1}} \sin \phi \frac{d}{d \phi}[ & \left.\sin ^{2} \phi \frac{d \mathfrak{B}_{n}}{d \phi}\right]-\sin ^{2} \phi \cos \phi \frac{d \mathfrak{B}_{n}}{d \phi}-n^{2} \sin \phi \mathfrak{B}_{n} \\
= & -\frac{r_{2}}{r_{1}} \sin \phi \frac{d}{d \phi}\left[\frac{r_{2} \sin \phi}{E \delta}\left(N_{\theta n}-\nu N_{\phi n}\right)-\frac{r_{1} \sin \phi}{E \delta}\left(N_{\phi n}-\nu N_{\theta n}\right)\right] \\
& +\frac{\sin \phi \cos \phi}{E \delta}\left[r_{2}\left(N_{\theta n}-\nu N_{\phi n}\right)-r_{1}\left(N_{\phi n}-\nu N_{\theta n}\right)\right] \\
& +\frac{2 i n(1+\nu) r_{2} \sin \phi}{E \delta} N_{\theta \phi n} .
\end{aligned}
$$

If we use the geometrical relations (8.2) and the function $f(z)$ representing the meridian of the shell, this equation becomes simply

$$
\frac{d^{2} \mathfrak{B}_{n}}{d z^{2}}-\left(\frac{f^{\prime \prime \prime}}{f^{\prime \prime}}+\frac{f^{\prime}}{f}\right) \frac{d \mathfrak{B}_{n}}{d z}+n^{2} \frac{f^{\prime \prime}}{f} \mathfrak{B}_{n}=h_{n}(z),
$$

where

$$
\begin{aligned}
h_{n}(z) \equiv & -f f^{\prime \prime} \frac{d}{d z}\left[\frac{1}{E \delta}\left(N_{\theta n}-\nu N_{\phi n}\right)\right]-f^{\prime \prime} \frac{d}{d z}\left[\frac{1+f^{\prime 2}}{f^{\prime \prime} E \delta}\left(N_{\phi n}-\nu N_{\theta n}\right)\right] \\
& +\frac{f^{\prime}}{f E \delta}\left(1+f^{\prime 2}\right)\left(N_{\phi n}-\nu N_{\theta n}\right)-\frac{2 i n(1+\nu) f^{\prime \prime}}{E \delta}\left(1+f^{\prime 2}\right)^{1 / 2} N_{\theta \phi n} .
\end{aligned}
$$

The solution of (8) may be written in the form

$$
\mathfrak{B}_{n}=E_{n} \mathfrak{B}_{n 1}+F_{n} \mathfrak{B}_{n 2}+\mathfrak{B}_{n p},
$$

(38) So far as I know, the only general solution of these equations which has been exhibited explicitly before this present work is that for the sphere; see Flügge (1934), pp. 54-58. 
where $\mathfrak{B}_{n 1}$ and $\mathfrak{B}_{n 2}$ are two linearly independent solutions of the homogeneous equation associated with (8), $E_{n}$ and $F_{n}$ are arbitrary constants, and $\mathfrak{B}_{n p}$ is a particular integral. It is easy to verify that a particular integral is given by

$$
\mathfrak{B}_{n p}=\frac{f(z) f^{\prime \prime}(z)}{\mathfrak{B}(z)} \int_{a}^{z} \frac{h_{n}(\xi)}{f(\xi) f^{\prime \prime}(\xi)}\left[\mathfrak{B}_{n 1}(\xi) \mathfrak{B}_{n 2}(z)-\mathfrak{B}_{n 1}(z) \mathfrak{B}_{n 2}(\xi)\right] d \xi,
$$

where $\mathfrak{B}(z)$ is the Wronskian of $\mathfrak{B}_{n 1}$ and $\mathfrak{B}_{n 2}$, and $a$ is any convenient point.

When the functions $\mathfrak{B}_{n}$ have been found as solutions of (8), the equations (2), (3), (4) will be satisfied if we obtain $U_{n}, V_{n}, W_{n}$ from the formulas

$$
\begin{aligned}
V_{n} & =\left(1+f^{\prime 2}\right)^{-1 / 2 \mathfrak{B}_{n},} \\
W_{n} & =-\frac{1+f^{\prime 2}}{f^{\prime \prime}} \frac{d}{d z}\left[\left(1+f^{\prime 2}\right)^{-1 / 2 \mathfrak{B}_{n}}\right]-\frac{\left(1+f^{\prime 2}\right)^{3 / 2}}{f^{\prime \prime} E \delta}\left[N_{\phi n}-\nu N_{\theta n}\right], \\
i n U_{n} & =\frac{1}{f^{\prime \prime}} \frac{d \mathfrak{B}_{n}}{d z}+\frac{f}{E \delta}\left[N_{\theta n}-\nu N_{\phi n}\right]+\frac{1+f^{\prime 2}}{f^{\prime \prime} E \delta}\left[N_{\phi n}-\nu N_{\theta n}\right] .
\end{aligned}
$$

Unfortunately the solution we have given here is not so convenient as that given in $\S 11$ for the equilibrium equations, for while there it was the complete solution which was of greatest interest, here it is the rather nasty particular integral.

The boundary conditions to be imposed on the displacements will usually be their vanishing at some support. Since in general the apex of a closed dome will not admit a membrane state of stress the behavior of the displacements near it will not be of interest. Should one desire, however, to investigate the displacements near a singularity, he could easily do so by methods similar to those of $\$ 15$. We shall merely notice that the exponents $\gamma_{n 1}$ and $\gamma_{n 2}$ of the solutions $\mathfrak{B}_{n 1}$ and $\mathfrak{B}_{n 2}$ near the apex of a closed dome of type (15.4) are given by the formulas

$$
\begin{aligned}
& \gamma_{n 1}=\left\{2 \mu-1+\left[1-4 \mu(\mu-1)\left(n^{2}-1\right)\right]^{1 / 2}\right\} / 2, \\
& \gamma_{n 2}=\left\{2 \mu-1-\left[1-4 \mu(\mu-1)\left(n^{2}-1\right)\right]^{1 / 2}\right\} / 2
\end{aligned}
$$

the radicals are the same as those in the exponents (15.5) of $\mathfrak{U}_{n 1}$ and $\mathfrak{U}_{n 2}$.

The form of the equation ( 8 ) indicates that the displacements may become infinite not only at singularities of the shell but also at points of inflection of the meridians or at points where the middle surface is perpendicular to the axis of revolution, and hence that a membrane state of stress may not exist near such points. Now in many cases by making $F_{n}$ zero in (10) we can find a displacement distribution which remains finite near such points. By this choice of constants we have effectively prescribed a certain displacement at the supports; then for such a displacement, and such a displacement only, may there be a membrane state of stress valid near the point in question. Such a state of affairs is not unexpected, for we have seen before that whether a 
given solution of the membrane equilibrium equations represents a membrane state of stress, that is, whether it gives a correct first approximation to the stress resultants in a given region, will depend on the particular one out of the many corresponding displacement distributions which is chosen. The criteria for such choices we could easily work out, but they are not sufficiently interesting to be worth the space demanded.

Before leaving the general subject of displacements, we should mention the elegant method of Flügge for finding the displacements of particular points $\left({ }^{39}\right)$, which, while of restricted applicability, often leads to the result more quickly than the general methods outlined here.

\section{EXAMPLES}

19. An indirect method of solution of the stress equation. The complete stress functions $\mathfrak{U}_{n c}{ }^{\left({ }^{40}\right)}$ satisfy the equation

$$
\mathfrak{u}_{n c}^{\prime \prime}+\frac{f^{\prime \prime}}{f}\left(n^{2}-1\right) \mathfrak{u}_{n c}=0 .
$$

We may write this equation in the form of a system:

$$
\begin{aligned}
f^{\prime \prime} / f & =A^{2} p(z), \\
\mathfrak{U}_{n c}^{\prime \prime} / \mathfrak{U}_{n c} & =-A^{2}\left(n^{2}-1\right) \dot{p}(z),
\end{aligned}
$$

where $p(z)$ is some function of $z$. Since (2) and (3) are of exactly the same type, the complete stress functions $\mathfrak{U}_{n c}$ may be obtained by replacing $A^{2}$ by $-A^{2}\left(n^{2}-1\right)$ in the general equation of the family of meridians satisfying (2), the process being subject to the proper alteration in the case when this change produces or destroys an integer exponent difference. By choosing functions $p(z)$ for which equation (2) is easily integrable, we may find the equations of many doubly infinite families of surfaces for which the equations may be solved exactly in terms of familiar functions; in the succeeding sections we shall enumerate and apply some of these results.

Equations (2) and (3) show conversely that if we have the complete stress functions for a surface with meridian $f$, they are also complete stress functions for any surface with a meridian belonging to the family

$$
r=f\left[A+B \int d z / f^{2}\right],
$$

where $A$ and $B$ are arbitrary constants, since if $f$ satisfies (2), so does any function $r$ included in the family (4). For example, the complete stress functions valid for the sphere, for which $f=\left(a^{2}-z^{2}\right)^{1 / 2}$, are equally valid for any surface whose meridian is of form

(39) Flügge (1934), pp. 59-63.

$\left({ }^{(0)}\right)$ We are using the terminology and notations of $\$ 11$. 


$$
r=\left(a^{2}-z^{2}\right)^{1 / 2}\left[A+B \operatorname{tnh}^{-1}(z / a)\right],
$$

in particular for spheroids and for hyperboloids. The solution given originally by $\mathrm{H}$. Reissner $\left({ }^{41}\right)$ for the sphere may be put in the form $\left({ }^{42}\right)$

$$
\mathfrak{u}_{n c}=\left(a^{2}-z^{2}\right)^{1 / 2}\left[C_{n}\left(\frac{a-z}{a+z}\right)^{n / 2}+D_{n}\left(\frac{a+z}{a-z}\right)^{n / 2}\right] .
$$

From these complete stress functions we may construct, with the aid of (11.11) and (11.13), the general solutions of the equilibrium equations for any member of the family (5). It should be remarked that this result holds only after we have expressed $\mathfrak{U}_{n c}$ in terms of $z$. For a sphere, the complete stress functions (6) may be written in the form in which they were originally given:

$$
\mathfrak{U}_{n c}=\sin \phi\left[C_{n} \tan ^{n}(\phi / 2)+D_{n} \cot ^{n}(\phi / 2)\right],
$$

but this representation is no longer correct for the other members of the family (5), since the relation between $\phi$ and $z$ is a function of $f$.

The value of the stress functions is greater than that of a mere device to solve the partial differential equations of equilibrium. $\mathfrak{U}_{n c}$ is much more intimately connected with the surface than are the stresses; no two surfaces in general lead to the same solutions of the equations of equilibrium, but any complete stress function is the common property of a doubly infinite family of surfaces. It is probably unnecessary to remark that the reason that two different surfaces having the same complete stress functions will nevertheless have different stress resultants is that the relations (11.13) which deduce the stress resultants from the stress functions are themselves functions of $f$.

20. The displacement equation. Unfortunately there seems to be no such simple method of finding solutions for (18.8). We may observe, however, that if $\mathfrak{B}_{n c}$ is the complete solution of (18.8) for a surface with meridian $f$, it is also the complete solution for surfaces with meridian $A f$, where $A$ is any constant.

Thus for example, from the solution of Rayleigh for the sphere $\left({ }^{43}\right)$,

$$
\mathfrak{B}_{n c}=E_{n} \tan ^{n}(\phi / 2)+F_{n} \cot ^{n}(\phi / 2),
$$

we may find the displacements of spheroids and hyperboloids with meridian $f=A\left(a^{2}-z^{2}\right)^{1 / 2}$ by expressing the result in terms of $z$ :

$$
\mathfrak{B}_{n c}=E_{n}\left(\frac{a-z}{a+z}\right)^{n / 2}+F_{n}\left(\frac{a+z}{a-z}\right)^{n / 2} \text {. }
$$

From (2), with the aid of (18.11) and (18.12), we may construct the general solutions of the displacement equations for spheroids and hyperboloids.

(11) H. Reissner (1912); Flügge (1934), pp. 38-39.

(42) This simple result is to be contrasted to that of Pucher (1938) (for the case when $B=0$ only), involving hypergeometric functions.

(4) Flügge (1934), p. 57. 
Our choice of displacement functions has been a happy one, for while in general under the same load distribution no two surfaces will have the same displacement distribution, each complete displacement function is the common property of an infinite family of surfaces.

We notice that the equation for the displacement functions usually has at least one more singularity than that for the stress functions. Thus if the stress functions are Bessel functions, the displacement functions will be hypergeometric functions; if the stress functions are hypergeometric functions, the displacement functions will be Heun functions, and so on.

21. Bessel and exponential functions; paraboloids. A natural choice for $p(z)$ in (19.2) is some power of $z$; we may write

$$
f^{\prime \prime}+A^{2}(z+a)^{1 / m-2} f=0,
$$$$
m \neq 0,
$$

as the equation for the meridians. Its integral family is easily shown to be

$$
f=(z+a)^{1 / 2} B_{m}\left[2 m A(z+a)^{1 /(2 m)}\right],
$$$$
m \neq 0,
$$

where we have put $B_{m}[x]$ as a convenient notation for the general solution of Bessel's equation in $x$ of order $m$. Then for the family (2) we shall have the complete stress functions

$$
\mathfrak{U}_{n c}=(z+a)^{1 / 2} B_{m}\left[2 m i A\left(n^{2}-1\right)^{1 / 2} .(z+a)^{1 /(2 m)}\right] .
$$

We notice that if $|n|>1$ the complete stress functions for a surface whose meridian is a combination of Bessel functions of the first kind are combinations of Bessel functions of the second kind, and vice versa. The solution (3) breaks down when $n= \pm 1$, and is not of interest when $n=0$; we shall observe this same phenomenon, naturally enough; with the other solutions in this chapter, and we notice now once and for all that if we wish solutions involving terms in which $n=0$ or \pm 1 we should resort for them to (12.1) and (14.1).

When $m=1 / 2$, the formulas (2) and (3) may be put into the form

$$
\begin{gathered}
f=A \sin (a z+b)+B \cos (a z+b), \\
\mathfrak{U}_{n c}=C_{n} \operatorname{sh}\left[\left(n^{2}-1\right)^{1 / 2}(a z+b)\right]+D_{n} \operatorname{ch}\left[\left(n^{2}-1\right)^{1 / 2}(a z+b)\right] .
\end{gathered}
$$

For the members of the family (4) for which $B=0$ it is easy also to find the complete displacement functions :

$$
\begin{aligned}
\mathfrak{B}_{n c}= & E_{n} F\left(-1+i\left(n^{2}-1\right)^{1 / 2},-1-i\left(n^{2}-1\right)^{1 / 2} ;-1 / 2 ; \sin ^{2}[(a z+b) / 2]\right) \\
& +F_{n} \sin ^{3}[(a z+b) / 2] F\left(1 / 2+i\left(n^{2}-1\right)^{1 / 2},\right. \\
& \left.1 / 2-i\left(n^{2}-1\right)^{1 / 2} ; 5 / 2 ; \sin ^{2}[(a z+b) / 2]\right),
\end{aligned}
$$

where $F(\alpha, \beta ; \gamma ; x)$ is the hypergeometric function. A similar result holds for the other half of the family (4). For an example of a boundary problem involving a dome with a meridian belonging to the family (4), see $\$ 24$. For the subfamily of (4) for which 
(7)

$$
f=A e^{a s}
$$

the complete displacement functions are

$$
\begin{aligned}
\mathfrak{B}_{ \pm 1, c} & =e^{a z}\left[E_{1}+F_{1 z}\right], \\
\mathfrak{B}_{n c} & =e^{a z}\left[E_{n} \cos \left(a\left(n^{2}-1\right)^{1 / 2} z\right)+F_{n} \sin \left(a\left(n^{2}-1\right)^{1 / 2} z\right)\right], \quad n \neq \pm 1
\end{aligned}
$$

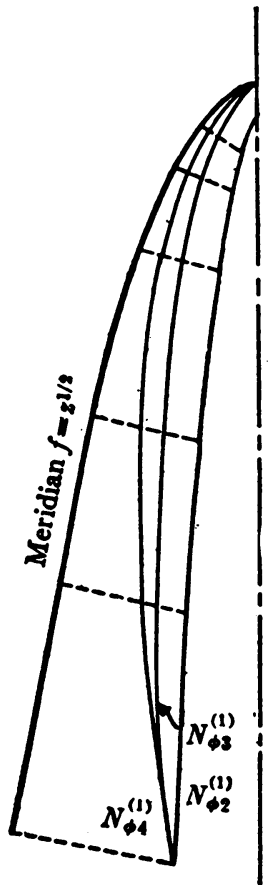

Fig. 8.

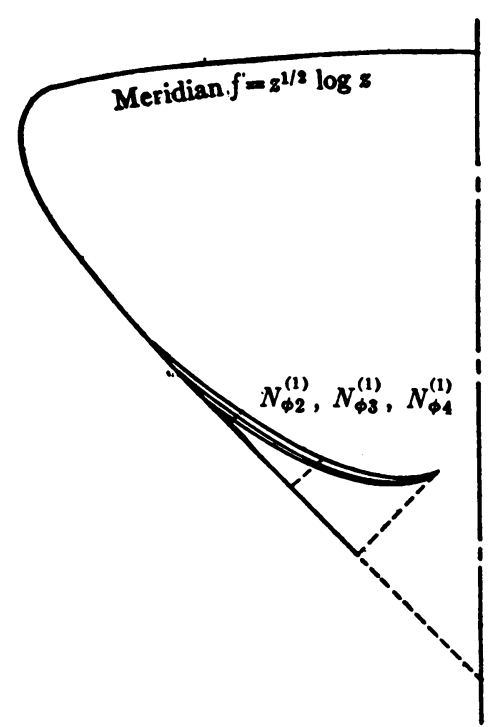

Fig. 9.

$N_{\phi \infty}^{(2)}$, constrained to unit value at the base, is read normally to the meridian.

If $m$ becomes infinite in (2), from the form of (1) we can see that our family of meridians becomes

$$
\begin{array}{ll}
f=A(z+a)^{\mu}+B(z+a)^{1-\mu}, & \mu \neq 1 / 2, \\
f=(z+a)^{1 / 2}[A+B \log (z+a)], & \mu=1 / 2,
\end{array}
$$

for which the complete stress functions are given by the formula

$$
\mathfrak{U}_{n c}=(z+a)^{1 / 2}\left[C_{n}(z+a)^{q(n)}+D_{n}(z+a)^{-q(n)}\right],
$$

where $[q(n)]^{2} \equiv 1 / 4-\mu(\mu-1)\left(n^{2}-1\right)$. This result is exactly what we should expect from the exponents (15.5). We may gain a qualitative estimate of these solutions from Fig. 8 and Fig. 9, which give plots of $N_{\phi n}^{(1)}$, when $n=2,3,4$, for two meridians included in the family (9), the parabola $f=z^{1 / 2}$, and the top-shaped curve $f=z^{1 / 2} \log z$, the value, which is prescribed to be unity at the 
lower edge, being read along the normal to the meridian.

For the sub-family of (9) for which $B=0$, it is easy to find the complete displacement functions :

$$
\mathfrak{B}_{n 0}=(z+a)^{\mu-1 / 2}\left[E_{n}(z+a)^{q(n)}+F_{n}(z+a)^{-q(n)}\right],
$$

where $q(n)$ is the same function of $n$ which was defined for the stress functions (10). The complete displacement functions for the meridians

$$
f=A(z+a)^{1 / 2} \log (z+a)
$$

are given by the equation

$$
\mathfrak{B}_{n c}=\log (z+a)\left\{E_{n}\left[\operatorname{ch} \log (z+a)^{n / 2}-\frac{2 \operatorname{sh} \log (z+a)^{n / 2}}{n \log (z+a)}\right]\right.
$$

$$
\left.+F_{n}\left[\operatorname{sh} \log (z+a)^{n / 2}-\frac{2 \operatorname{ch} \log (z+a)^{n / 2}}{n \log (z+a)}\right]\right\} \text {. }
$$

One of the surfaces easiest to treat in the membrane theory is the paraboloid, for which

$$
f=2 A(a-z)^{1 / 2} .
$$

Let us find the membrane stresses in such a shell, subject to its own weight, supported uniformly on $m$ symmetrical columns at its base $z=0$. First we shall need a solution for the shell supported uniformly at each point of its base; evaluating the constant of integration in (12.4) in accordance with conditions (15.3), we find that

$$
\begin{aligned}
& N_{\phi 0}=-2 p\left(a+A^{2}-z\right)^{1 / 2}\left[\left(a+A^{2}-z\right)^{3 / 2}-A^{3}\right] /[3(a-z)], \\
& N_{\theta 0}=-A N_{\phi 0} /\left(a+A^{2}-z\right)-2 A^{2} p, \quad N_{\theta \phi 0}=0 .
\end{aligned}
$$

Upon this solution we shall superpose a second, for which $X=0, Y=0, Z=0$, satisfying the conditions

$$
\left.N_{\phi}\right|_{z=0}=\left\{\begin{array}{l}
-P(\pi-m \epsilon) /(m \epsilon) \text { at the supports, } \\
P \text { elsewhere, }
\end{array}\right.
$$

where $2 \epsilon$ is the angular width of the supporting columns, and $P=-\left.N_{\phi 0}\right|_{z=0}$, or

$$
P=2 p\left(a+A^{2}\right)^{1 / 2}\left[\left(a+A^{2}\right)^{3 / 2}-A^{3}\right] /(3 a) .
$$

The boundary stresses (16) form an equilibrium system, and when $P$ is chosen by (17), the stress $N_{\phi}$ will vanish between the supports. $N_{\theta \phi}$ will not vanish everywhere between the supports, so we must suppose some sort of rigid ring is attached to the bottom of the shell to absorb the shear, or else we must regard our solution merely as a first approximation to which correcting terms from the bending theory should be added. The Fourier series for (16) is 


$$
\left.N_{\phi}\right|_{z=0}=-\frac{2 P}{\epsilon} \sum_{n=m, 2 m, 3 m, \ldots}^{\infty} \frac{\sin n \epsilon}{n} \cos n \theta,
$$

if we suppose the supports occupy the regions where $-\epsilon \leqq \theta \leqq \epsilon, 2 \pi / m-\epsilon \leqq \theta$ $\leqq 2 \pi / m+\epsilon, \cdots, 2(m-1) \pi / 2-\epsilon \leqq \theta \leqq 2(m-1) \pi / 2+\epsilon$. We now construct from the complete stress functions (10) a solution such that (18) will be satisfied; the boundary condition at the apex, as we saw in $\$ 15$, is $D_{n}=0$.

$$
N_{\phi}=-\frac{2 P a\left(a+A^{2}-z\right)^{1 / 2}}{\epsilon(a-z)\left(a+A^{2}\right)^{1 / 2}} \sum_{n=m, 2 m, \cdots}^{\infty} \frac{\sin n \epsilon}{n}\left(1-\frac{z}{a}\right)^{n / 2} \cos n \theta,
$$

(19) $\quad N_{\theta}=-A^{2} N_{\phi} /\left(a+A^{2}-z\right)$,

$$
N_{\theta \phi}=-\frac{2 P a A}{\epsilon(a-z)\left(a+A^{2}\right)^{1 / 2}} \sum_{n=m, 2 m}^{\infty} \ldots \frac{\sin n \epsilon}{n}\left(1-\frac{z}{a}\right)^{n / 2} \sin n \theta .
$$

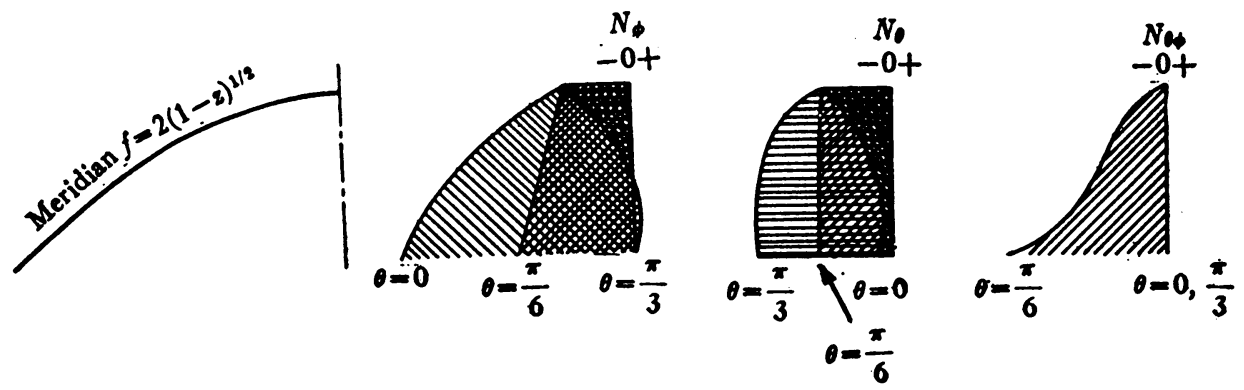

FIG. 10.

For the case when $m=3, \epsilon=\pi / 6, a=1, A=1$, a plot of this solution, with (13) superposed, at the meridians $\theta=0, \pi / 6, \pi / 3$, that is, at the middle of the supports, at the edges of the supports, and midway between the supports, is given in Fig. 10.

When the supports are very narrow, we can simplify the calculation by. letting $\epsilon$ approach 0 in (19). The series then become geometric series for certain values of $\theta$; in particular,

(20)

$$
\begin{gathered}
\left.N_{\phi}\right|_{\theta=0}=-\frac{2 P a\left(a+A^{2}-z\right)^{1 / 2}}{(a-z)\left(a+A^{2}\right)^{1 / 2}} \frac{\left(1-\frac{z}{a}\right)^{m / 2}}{1-\left(1-\frac{z}{a}\right)^{m / 2}}, \\
\left.N_{\phi}\right|_{\theta=x / 2 m}=\frac{2 P a\left(a+A^{2}-z\right)^{1 / 2}}{(a-z)\left(a+A^{2}\right)^{1 / 2}} \frac{\left(1-\frac{z}{a}\right)^{m}}{1+\left(1-\frac{z}{a}\right)^{m}},
\end{gathered}
$$


(20)

$$
\begin{gathered}
\left.N_{\phi}\right|_{\theta=\pi / m}=\frac{2 P a\left(a+A^{2}-z\right)^{1 / 2}}{(a-z)\left(a+A^{2}\right)^{1 / 2}} \frac{\left(1-\frac{z}{a}\right)^{m / 2}}{1+\left(1-\frac{z}{a}\right)^{m / 2}}, \\
\left.N_{\theta \phi}\right|_{\theta=\pi / 2 m}=-\frac{2 P a A}{(a-z)\left(a+A^{2}\right)^{1 / 2}} \frac{\left(1-\frac{z}{a}\right)^{m / 2}}{1+\left(1-\frac{z}{a}\right)^{m}} .
\end{gathered}
$$

For the case when $m=3, a=1, A=1$, these results are plotted in Fig. 11 to the same scale as Fig. 10.

The reader may note that the general considerations of existence and value at the apex of $\$ 15$ are easily verified in this example. In particular, when

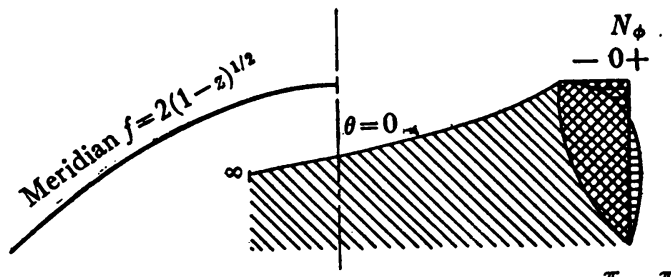

$$
\theta=\frac{\pi}{6}, \frac{\pi}{3}
$$

FIG. 11.

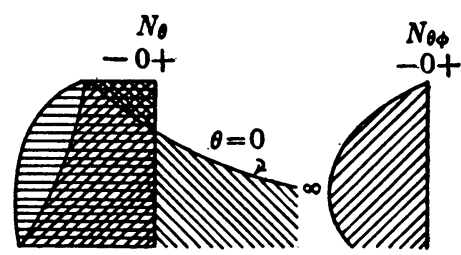

$\theta=\frac{\pi}{3}, \frac{\pi}{6}$

$m=2$, the stress resultants will be finite but not zero at the apex, but when $m>2$ all but the part due to (15) will vanish at the apex.

To compute the displacements corresponding to the solution (19), we begin with the complete displacement functions

$$
\mathfrak{B}_{n c}=E_{n}(a-z)^{n / 2}+F_{n}(a-z)^{-n / 2}
$$

which are given by (11). Since the apex is not a singularity of (17), we shall expect all displacements to remain finite there; hence $F_{n}=0$ is a boundary condition. To (21) must be added a particular integral from (18.11):

$$
\begin{array}{r}
\mathfrak{B}_{n p}=(1 / n) \int^{z} h_{n}(\xi)(a-\xi)\left[(a-\xi)^{n / 2}(a-z)^{-n / 2}\right. \\
\left.-(a-\xi)^{-n / 2}(a-z)^{n / 2}\right] d \xi .
\end{array}
$$

The function $h_{n}(z)$, given by (18.9) from the stresses (19), although even when $\delta=$ const. it is rather long, can be computed by straightforward calculations and the quadrature (22) can be evaluated with elementary functions, but the result is too cumbersome for us to tarry over it here. The constant $E_{n}$ should be evaluated by the condition $U=0$ when $z=0$. Upon this solution 
should be superposed one corresponding to (15), which is easily computed.

It is interesting to compare these results with those for the corresponding problem for the top-shaped shell whose meridian is

$$
f=z^{1 / 2} \log z \text {. }
$$

Unfortunately the integral (12.4) for the everywhere supported shell is not elementary, and is indeed quite hard to handle. When the dome is supported at $z=z_{0}, z_{0}<1$, the part of the solution corresponding to (19) is

(24)

$$
\begin{aligned}
& N_{\phi}=-\frac{2 P}{\epsilon} \frac{\left[z+(1+\log z / 2)^{2}\right]^{1 / 2}}{\left[z_{0}+\left(1+\log z_{0} / 2\right)^{2}\right]^{1 / 2}} \frac{z_{0}}{z} \frac{\left(\log z_{0}\right)^{2}}{(\log z)^{2}} \\
& N_{\theta}=-\frac{4 z^{3}}{z+(1+\log z / 2)^{2}} N_{\phi}^{\infty} \frac{\sin n \epsilon}{n}\left(\frac{z}{z_{0}}\right)^{n / 2} \cos n \theta, \\
& N_{\theta \phi}=\frac{2 P}{\epsilon} \frac{z_{\theta}\left(\log z_{0}\right)^{2}}{z\left[z_{0}+\left(1+\log z_{0} / 2\right)^{2}\right]^{1 / 2}} \\
& \cdot \sum_{n=m, 2 m,}^{\infty} \frac{\sin n \epsilon}{n}\left[1 / 2-(\log z)^{-2 / n]}\left(\frac{z}{z_{0}}\right)^{n / 2} \sin n \theta .\right.
\end{aligned}
$$

The family (2) does not contain any Bessel functions of zero order, but a different analysis will yield a result for them, too. The integral of the equation

$$
f^{\prime \prime}+a^{2} A^{2}\left[1+(a z+b)^{-2} / 4\right] f=0
$$

is

$$
f=(a z+b)^{1 / 2} B_{p}[A(a z+b)],
$$

where $p \equiv-\left(1-A^{2}\right)^{1 / 2} / 2$; accordingly the complete stress functions for this family are given by the equation

$$
\mathfrak{u}_{n c}=(a z+b)^{1 / 2} B_{m}\left[i A\left(n^{2}-1\right)^{1 / 2}(a z+b)\right],
$$

where $m \equiv\left[1+A^{2}\left(n^{2}-1\right)\right]^{1 / 2} / 2$. The case of greatest interest is that for which $A=1$, namely, that for which

$$
f=(a z+b)^{1 / 2}\left[A J_{0}(a z+b)+B Y_{0}(a z+b)\right] .
$$

For this family

$$
\begin{aligned}
& \mathfrak{U}_{2 n, c}=(a z+b)^{1 / 2}\left\{C_{2 n} I_{n}\left[\left(4 n^{2}-1\right)^{1 / 2}(a z+b)\right]\right.\left.\cdot D_{2 n} K_{n}\left[\left(4 n^{2}-1\right)^{1 / 2}(a z+b)\right]\right\} \\
& \mathfrak{U}_{2 n+1, c}=(a z+b)^{1 / 2}\left\{C_{2 n+1} I_{n+1 / 2}\left[2\left(n^{2}+n\right)^{1 / 2}(a z+b)\right]\right. \\
&\left.+D_{2 n+1} I_{-n-1 / 2}\left[2\left(n^{2}+n\right)^{1 / 2}(a z+b)\right]\right\}
\end{aligned}
$$


While a balloon is essentially a shell which derives a shape of its own rather than being rigidly constricted to any form, the meridian

$$
f=z^{1 / 2} J_{0}(z)
$$

is not unlike the shape often assumed (see Fig. 12). Let us investigate a boundary problem which might be considered that of a balloon subject to the

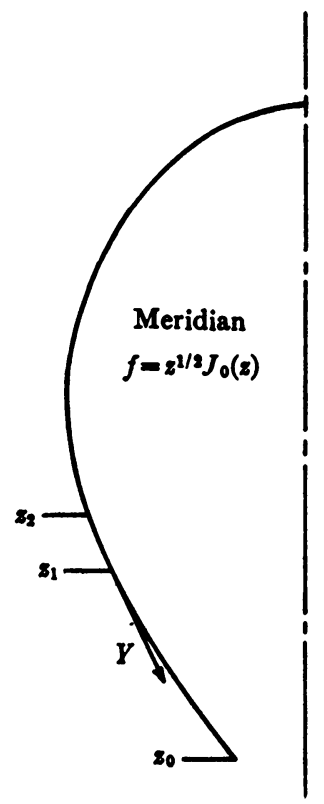

FIG. 12.

pull of $2 m$ symmetrically attached ropes acting on that part of the bag between $z=z_{1}$ and $z=z_{2}$. Then

(31) $\quad \boldsymbol{Y}=\left\{\begin{array}{lr}F \text { on } 2 m \text { symmetrical regions of angular width } 2 \epsilon \\ 0 \text { elsewhere } \\ 0, & z>z_{1} \text { or } z<z_{2} .\end{array}\right.$

Let the boundary conditions be the vanishing of $N_{\phi}$ at the lower edge, $z=z_{0}$. We may represent the load (31) in the form

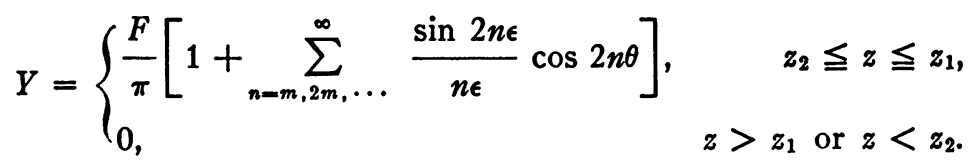

A set of stress functions satisfying the boundary condition at $z=0$ (see $\S 15$ ) is given by the formula 


$$
\mathfrak{U}_{2 n}=z^{1 / 2}\left(C_{n} I_{n}\left[\left(4 n^{2}-1\right)^{1 / 2} z\right]+\int_{0}^{z} F(z, t) d t\right), \quad|n|>0,
$$

where

$$
\begin{aligned}
& F(z, t) \equiv t^{1 / 2} g_{2 n}(t)\left\{I_{n}\left[\left(4 n^{2}-1\right)^{1 / 2} z\right] K_{n}\left[\left(4 n^{2}-1\right)^{1 / 2} t\right]\right. \\
& \left.-I_{n}\left[\left(4 n^{2}-1\right)^{1 / 2} t\right] K_{n}\left[\left(4 n^{2}-1\right)^{1 / 2} z\right]\right\}, \\
& g_{2 n}(t)=\left\{\begin{array}{rr}
\frac{3 F \sin 2 n \epsilon}{\pi n \epsilon}\left[\frac{J_{0}(t)}{2}-t J_{1}(t)\right] J_{0}(t), & z_{2} \leqq t \leqq z_{1}, \\
0, & t<z_{2} \text { or } z_{0} \geqq t>z_{1} .
\end{array}\right.
\end{aligned}
$$

Then

$$
\mathfrak{U}_{2 n}=\left\{\begin{array}{lr}
z^{1 / 2} C_{2 n} I_{n}\left[\left(4 n^{2}-1\right)^{1 / 2} z\right], & z<z_{2}, \\
z^{1 / 2}\left(C_{2 n} I_{n}\left[\left(4 n^{2}-1\right)^{1 / 2} z\right]+\int_{z_{2}}^{z} F(z, t) d t\right), & z_{2} \leqq z \leqq z_{1}, \\
z^{1 / 2}\left(C_{2 n} I_{n}\left[\left(4 n^{2}-1\right)^{1 / 2} z\right]+\int_{z_{2}}^{z_{1}} F(z, t) d t\right), & z_{0} \geqq z \geqq z_{1} .
\end{array}\right.
$$

The condition $N_{\phi}=0$ at $z=z_{0}$ yields the value of $C_{2 n}$ :

$$
C_{2 n}=-\int_{z_{2}}^{z_{1}} F(z, t) d t / I_{n}\left[\left(4 n^{2}-1\right)^{1 / 2} z_{0}\right] .
$$

These results dispose of the terms for which $n \neq 0$ in (32); there remains the term in which $n=0$ :

$$
\mathfrak{U}_{0}=\left\{\begin{array}{lr}
\frac{F}{\pi} z^{1 / 2} J_{0}(z) \int_{z_{2}}^{z_{1}} \xi^{1 / 2} J_{0}(\xi) d \xi, & z<z_{2}, \\
\frac{F}{\pi} z^{1 / 2} J_{0}(z) \int_{z}^{z_{1}} \xi^{1 / 2} J_{0}(\xi) d \xi, & z_{2} \leqq z \leqq z_{1} . \\
0, & z_{1} \leqq z \leqq z_{0},
\end{array}\right.
$$

as can easily be verified from the integral (12.1). The load (31) does not form an equilibrium system; its resultant downward force should be cancelled by a symmetrical upward force due to the lift of the atmosphere.

22. A transformation. Conicoids. Sometimes the knowledge of the solution for a surface with meridian $f$ will lead us to the solutions for some other surface with meridian $g$. Say that

$$
f^{\prime \prime} / f=A^{2} p(z),
$$

and say we make the substitution $z=\phi(x)$. The question then is, can we find some $g, g=g(f, x)$, such that $g$ satisfies an equation of the type 


$$
\frac{d^{2} g}{d x^{2}} / g=B^{2} q(x)
$$

say? Of course, neither $p, \phi$, nor $q$ is to be a function of $A$ or $B$. A straightforward analysis in the theory of transformations of linear differential equations shows that such a $g$ can be found only for the transformation $\phi(x)$ $=(a x+b) /(c x+d)$, and then

$$
g=(c x+d) f\left(\frac{a x+b}{c x+d}\right) .
$$

Then from the discussion in the beginning of $\$ 19$ we may conclude that if the $\mathfrak{U}_{n c}(z)$ are the complete stress functions for a surface with meridian $f(z)$, for a surface with meridian (3) the complete stress functions are

$$
(c x+d) \mathfrak{u}_{n c}\left(\frac{a x+b}{c x+d}\right) .
$$

In particular, we may verify and extend an earlier result by supposing that $c=0$ and $d=1$; it follows that if the $\mathfrak{U}_{n c}$ are the complete stress functions for $f(x)$, then the functions $\mathfrak{u}_{n c}(a x+b)$ are the complete stress functions for $A f(a x+b)$.

From (21.4) and (21.5) we have at once that for surfaces such that

$$
f=(c x+d)\{A \sin [(a x+b) /(c x+d)]+B \cos [(a x+b) /(c x+d)]\},
$$

the complete stress functions are given by the equation

$$
\begin{aligned}
\mathfrak{u}_{n c}=(c x+d)\left\{C_{n} \operatorname{sh}\left[\left(n^{2}-1\right)^{1 / 2}(a x+b) /(c x+d)\right]\right. \\
\left.+D_{n} \operatorname{ch}\left[\left(n^{2}-1\right)^{1 / 2}(a x+b) /(c x+d)\right]\right\} .
\end{aligned}
$$

From our solution for the paraboloid (the case when $\mu=1 / 2$ in (21.9) and (21.10)), by applying the bilinear transformation, we may see, after a rearrangement of constants, that for a surface with meridian

$$
f=(a-z)^{1 / 2}(b-z)^{1 / 2}[A+B \log ((a-z) /(b-z))]
$$

the complete stress functions are given by the expression

$$
\mathfrak{u}_{n c}=(a-z)^{1 / 2}(b-z)^{1 / 2}\left[C_{n}\left(\frac{a-z}{b-z}\right)^{n / 2}+D_{n}\left(\frac{b-z}{a-z}\right)^{n / 2}\right] .
$$

The family (6), when $b=-a$, is simply that of spheres, spheroids, hyperboloids, and so on, which we found already (19.5); it contains various ovoids also. For the subfamily of (6) for which $B=0$, namely the conicoids and ovoids only, it is not difficult to find the complete displacement functions from (18.8): 
(8)

$$
\mathfrak{B}_{n c}=E_{n}\left(\frac{a-z}{b-z}\right)^{n / 2}+F_{n}\left(\frac{b-z}{a-z}\right)^{n / 2} .
$$

This result contains (20.2) as a special case. For graphs of the stress coefficients $N_{\phi m}^{(1)}$ derived from the solutions (7) for the sphere, two spheroids, and a hyperboloid of two sheets, see Figs. 13-16.

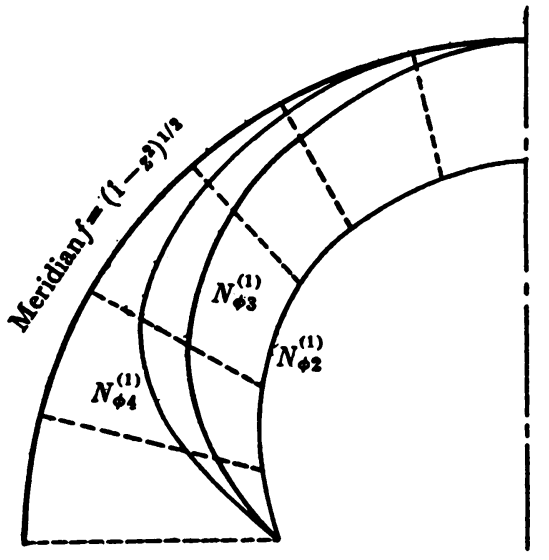

Fig. 13.

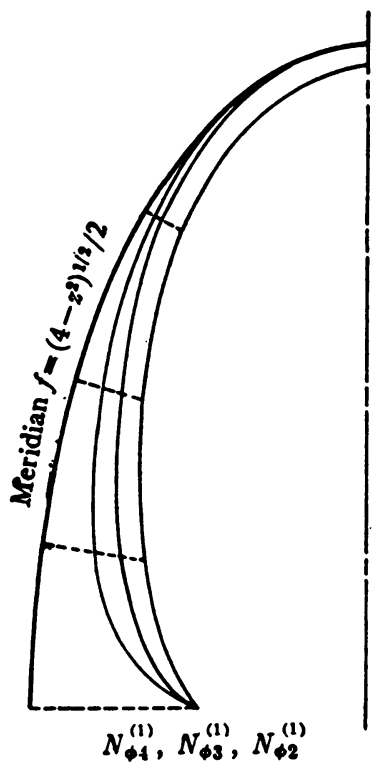

FIG. 14.

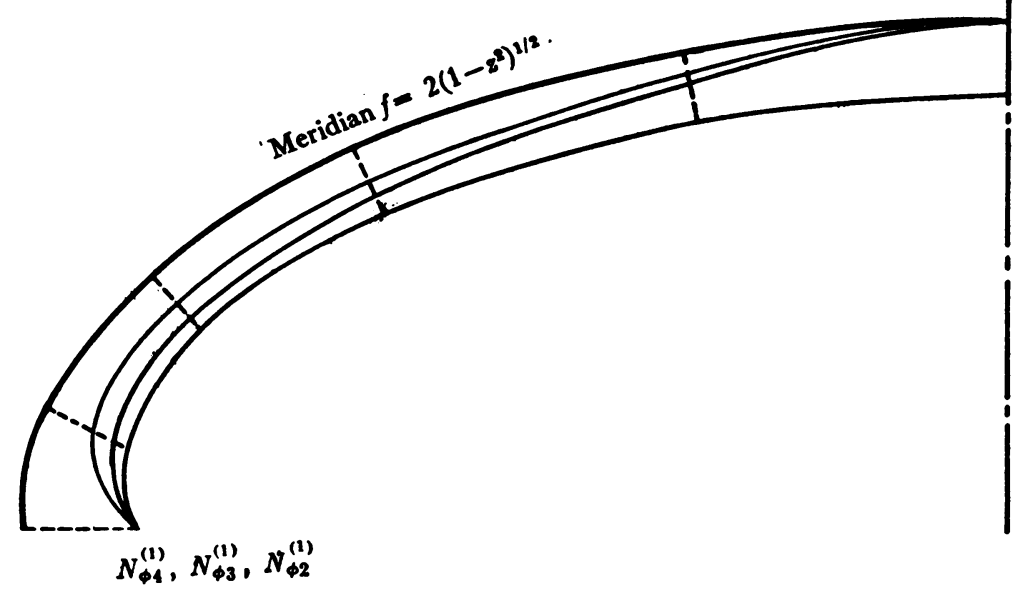

Fig. 15. 


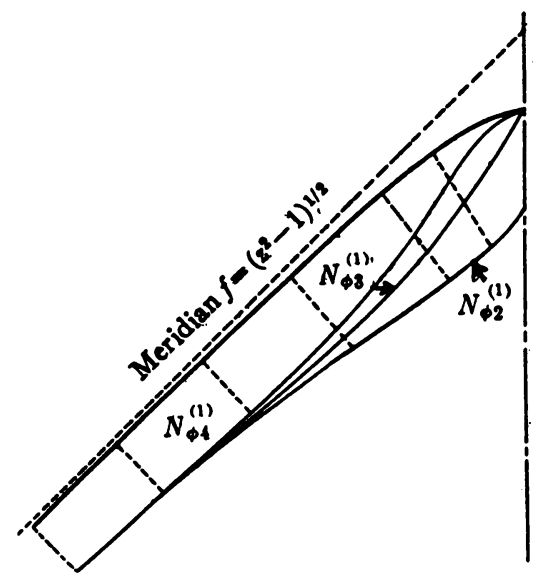

FIG. 16.

23. Two general families of surfaces. Most of the results hitherto considered are special cases of the two following.

Consider the equation

$$
f^{\prime \prime}+\frac{A^{2}\left(1-\beta^{2}\right)(a-b)^{2}}{4(z-a)^{2}(z-b)^{2}} f=0 .
$$

If we define $\lambda$ and $\gamma$ by the formulas

(2) $\lambda \equiv A / 2+\left[\left(\beta^{2}-1\right) A^{2}+1\right]^{1 / 2} / 2, \quad \gamma \equiv A / 2-\left[\left(\beta^{2}-1\right) A^{2}+1\right]^{1 / 2} / 2$, and make the substitution

$$
f=(z-a)^{(1-\lambda-\gamma) / 2}(z-b)^{(1+\lambda+\gamma) / 2} \eta,
$$

then (1) assumes the form

$$
\frac{d^{2} v}{d z^{2}}+\left\{\frac{1-\lambda-\gamma}{z-a}+\frac{1+\lambda+\gamma}{z-b}\right\} \frac{d v}{d z}+\frac{\lambda \gamma(a-b)^{2}}{(z-a)^{2}(z-b)^{2}} v=0
$$

Hence the integral of (1) is given by the expression $\left({ }^{44}\right)$

$$
f=(z-a)^{(1-\lambda-\gamma) / 2}(z-b)^{(1+\lambda+\gamma) / 2}\left[B\left(\frac{z-a}{z-b}\right)^{\lambda}+C\left(\frac{z-a}{z-b}\right)^{\gamma}\right],
$$

when $\lambda \neq \gamma$. Replacing $A^{2}$ by $-A^{2}\left(n^{2}-1\right)$ in $\lambda$ and $\gamma$ is equivalent to replacing $\lambda$ and $\gamma$ by $\lambda(n)$ and $\gamma(n)$ respectively, where

$$
\begin{aligned}
& \lambda(n)=i\left\{\lambda+\gamma+\left[(\lambda-\gamma)^{2}-n^{2}\left(n^{2}-1\right)^{-1 / 2}\right]^{1 / 2}\right\}\left(n^{2}-1\right)^{1 / 2} / 2, \\
& \gamma(n)=i\left\{\lambda+\gamma-\left[(\lambda-\gamma)^{2}-n^{2}\left(n^{2}-1\right)^{-1 / 2}\right]^{1 / 2}\right\}\left(n^{2}-1\right)^{1 / 2} / 2 .
\end{aligned}
$$

(4) Whittaker and Watson (1927), p. 208. 
Hence the solutions $\mathfrak{U}_{n c}$ for the five parameter family of surfaces (4) are obtained by replacing $\lambda$ and $\gamma$ by $\lambda(n)$ and $\gamma(n)$ respectively. This analysis of course must be modified when $\lambda=\gamma$, when the result turns out to be exactly what we have found earlier in equations (22.6), (22.7).

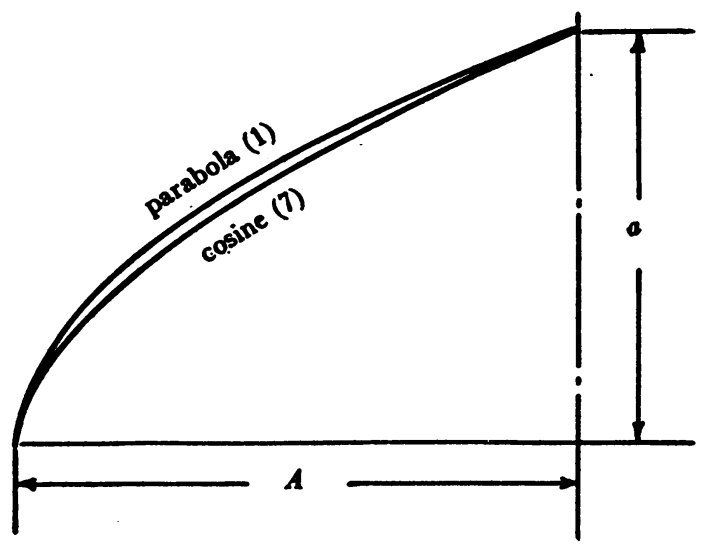

Frg. 17.

The equation

$$
f^{\prime \prime}+A^{2}\left[-a^{2}+b /(z-\alpha)+c /(z-\alpha)^{2}\right] f=0
$$

may be put into the form

$$
\frac{d^{2} f}{d y^{2}}+\left\{-1 / 4+p / y+\left(1 / 4-q^{2}\right) / y^{2}\right\} f=0,
$$

which is Whittaker's equation( $\left.{ }^{45}\right)$, if we choose $y, p, q$, and $r$ by the definitions

$$
y \equiv r(z-\alpha), \quad p \equiv b A / 2, \quad q \equiv\left(1 / 4-c A^{2}\right)^{1 / 2}, \quad r \equiv 2 a A .
$$

Replacing $A^{2}$ by $-A^{2}\left(n^{2}-1\right)$ in (6) is equivalent to replacing $p, q$, and $r$ by $p(n), q(n)$, and $r(n)$ respectively, where

$$
\begin{gathered}
p(n) \equiv i p\left(n^{2}-1\right)^{1 / 2}, \quad q(n) \equiv i q\left[n^{2}-1-n^{2} /\left(4 q^{2}\right)\right]^{1 / 2} \\
r(n) \equiv i r\left(n^{2}-1\right)^{1 / 2} .
\end{gathered}
$$

Accordingly if the meridian is included in the family

$$
f=B W_{p, q}[r(z-\alpha)]+C W_{-p, q}[-r(z-\alpha)],
$$

the complete stress functions $\mathfrak{u}_{n c}$ may be obtained by replacing $p, q$, and $r$ by $p(n), q(n)$, and $r(n)$, respectively, in (10). This analysis includes much of that of $\$ 21$ as special cases.

A result of equal generality could be written down when the meridian is a

(5) Whittaker and Watson (1927), pp. 337, 339-340, 343. 
hypergeometric function, but the formulas are too complicated to be useful. Some special cases are considered in the succeeding sections.

24. Two pointed domes. Let us consider the pointed dome whose meridian is the parabola

$$
f=\left(A / a^{2}\right)\left(a^{2}-z^{2}\right)
$$

(see Fig. 17). We shall first solve the problem of such a dome subject to its own weight and uniformly supported. A straightforward calculation with the integral (12.4) yields the solution

$$
\begin{aligned}
N_{\phi 0}= & -\frac{p \rho}{4\left(a^{2}-z^{2}\right)}\left[\frac{a^{4}}{A}\left(1+\frac{a^{2}}{16 A^{2}}\right) \log \frac{2 A z / a^{2}+\rho}{2 A / a+\alpha}\right. \\
& \left.+2 a^{2}\left(1+\frac{a^{2}}{16 A^{2}}\right)(z \rho-a \alpha)+\frac{a^{4}}{4 A^{2}}\left(a \alpha^{3}-z \rho^{3}\right)\right], \\
N_{\theta 0}= & \frac{2 A^{2}\left(a^{2}-z^{2}\right)}{a^{4}}\left[-p z-N_{\phi 0} / \rho^{2}\right],
\end{aligned}
$$

where for convenience we have used the abbreviations

$$
\rho \equiv\left(1+4 A^{2} z^{2} / a^{4}\right)^{1 / 2}, \quad \alpha \equiv\left(1+4 A^{2} / a^{2}\right)^{1 / 2} .
$$

When the shell is subject to "wind pressure" (14.2), the solution, given by the integral (14.1), may be shown to be

$$
\begin{aligned}
N_{\phi}= & \frac{F a^{6} \rho \cos \theta}{2 A\left(a^{2}-z^{2}\right)^{2}}\left[C(z-a)+\left(1+\frac{a^{2}}{8 A^{2}}\right) \cdot \frac{z}{a} \log \frac{2 A z / a^{2}+\rho}{2 A / a+\alpha}\right. \\
& +\left(1-\frac{3 a^{2}}{16 A^{2}}-\frac{a^{4}}{32 A^{4}}\right)(\rho-\alpha) \\
& \left.-\frac{a^{2}}{8 A^{4}}\left(a^{2} / 2+4 A^{2} / 3\right)\left(\rho^{3}-\alpha^{3}\right)+\frac{a^{4}}{80 A^{4}}\left(\rho^{5}-\alpha^{5}\right)\right],
\end{aligned}
$$

(4) $N_{\theta}=-\frac{A}{a^{2}}\left(a^{2}-z^{2}\right)\left[F \cos \theta+2 A N_{\phi} /\left(a^{2} \rho^{2}\right)\right]$,

$$
\begin{aligned}
N_{\theta \phi}= & -\frac{2 A \tan \theta}{a^{2} \rho} N_{\phi}-\frac{F a^{4} \sin \theta}{2\left(a^{2}-z^{2}\right)}\left[C+\frac{1}{a}\left(1+\frac{a^{2}}{8 A^{2}}\right) \log \frac{2 A z / a^{2}+\rho}{2 A / a+\alpha}\right. \\
& +\left(\frac{2 A}{a^{3}}+\frac{1}{4 a A}+\frac{4 A^{2}}{a^{4}}-\frac{3}{4 a^{2}}-\frac{1}{4 A^{2}}\right) \frac{z}{\rho} \\
& \left.+\left(\frac{1}{3 A^{2}}+\frac{4}{3 a^{2}}\right)(3 z \rho / 2)+\frac{z \rho^{3}}{4 A^{2}}\right]+\frac{2 A^{2}\left(a^{2}-z^{2}\right)}{a^{4} \rho} F \sin \theta,
\end{aligned}
$$


where

$$
\begin{aligned}
C= & -(1 / A \alpha)\left[2 A^{2} / a^{2}+1 / 4+4 A^{3} / a^{3}-3 A /(4 a)-a /(8 A)\right] \\
& +\frac{\alpha}{a}\left[1+3 a^{2} /\left(4 A^{2}\right)\right]+\alpha^{3} a /\left(4 A^{2}\right) .
\end{aligned}
$$

We now consider a problem which was treated by approximate methods by Flügge $\left({ }^{(6)}\right)$, namely that of a shell with meridian (1) supported on $m$ columns at $z=0$, as was the paraboloid of $\$ 21$. Flügge replaced the differential equations of equilibrium by difference equations, then computed the solutions by interpolation, separating the infinite from the finite portions of the solutions at the apex by taking means. Flügge's numerical result is that $N_{\phi}=0$ at $z=a$, but in $\$ 15$ we have seen that when $\mu=1$, which is the case in the present problem, $N_{\phi}$ must be infinite at the apex. Possibly the apex is no longer a singularity in the difference equations. It is easy to show that

(5) $\mathfrak{U}_{n 1}=\left(a^{2}-z^{2}\right) F\left(\left[3+i\left(8 n^{2}-9\right)^{1 / 2}\right] / 2,\left[3-i\left(8 n^{2}-9\right)^{1 / 2}\right] / 2 ; 2 ; 1 / 2-z /(2 a)\right)$.

$\mathfrak{U}_{n 2}$ we shall not wish. The complete stress functions may be expressed in any one of a variety of forms, as hypergeometric functions with argument $1-z^{2} / a^{2}$, Legendre or Gegenbauer functions, and so on, but we shall use the form (5) as a point of departure. In view of the discussions of $\$ 15$ a solution analogous to (21.19) valid sufficiently far from the apex is

$$
\begin{aligned}
N_{\phi}=-\frac{2 P a^{2}\left(1+4 A^{2} z^{2} / a^{4}\right)^{1 / 2}}{\epsilon\left(a^{2}-z^{2}\right)\left(1+4 A^{2} / a^{2}\right)^{1 / 2}} \sum_{n=m, 2 m, \ldots}^{\infty} \frac{\sin n \epsilon}{n} \\
\\
\quad \frac{F\left(\left[3+i\left(8 n^{2}-9\right)^{1 / 2}\right] / 2,\left[3-i\left(8 n^{2}-9\right)^{1 / 2}\right] / 2 ; 2 ; 1 / 2-z /(2 a)\right)}{F\left(\left[3+i\left(8 n^{2}-9\right)^{1 / 2}\right] / 2,\left[3-i\left(8 n^{2}-9\right)^{1 / 2}\right] / 2 ; 2 ; 1 / 2\right)} \cos n \theta,
\end{aligned}
$$

(6) $N_{\theta}=-\frac{2 A^{2}}{a^{4}} \frac{a^{2}-z^{2}}{\left(1+4 A^{2} z^{2} / a^{4}\right)} N_{\phi}$,

$$
\begin{aligned}
N_{\theta \phi}= & -\frac{2 P A}{a \epsilon\left(1+4 A^{2} / a^{2}\right)^{1 / 2}} \sum_{n=m, 2 m}^{\infty}, \ldots \\
& \sin n \epsilon \\
& \frac{F\left(\left[5+i\left(8 n^{2}-9\right)^{1 / 2}\right] / 2,\left[5-i\left(8 n^{2}-9\right)^{1 / 2}\right] / 2 ; 3 ; 1 / 2-z /(2 a)\right)}{F\left(\left[3+i\left(8 n^{2}-9\right)^{1 / 2}\right] / 2,\left[3-i\left(8 n^{2}-9\right)^{1 / 2}\right] / 2 ; 2 ; 1 / 2\right)} \sin n \theta,
\end{aligned}
$$

where $P$ is chosen so that $P=-\left.N_{\phi 0}\right|_{z=0}, N_{\phi \theta}$ being given by (2).

The hypergeometric function in the denominators is difficult to compute from the series, which converge very slowly when $n$ is large, but the formula $\left({ }^{47}\right)$

(16) Flügge (1934), pp. 47-49.

(47) Whittaker and Watson (1927), p. 298, exercise 13. 


$$
\begin{aligned}
F\left(\left[3+i\left(8 n^{2}-9\right)^{1 / 2}\right] / 2,\right. & {\left.\left[3-i\left(8 n^{2}-9\right)^{1 / 2}\right] / 2 ; 2 ; 1 / 2\right) } \\
= & \frac{\pi^{1 / 2}}{\Gamma\left(3 / 4+i\left(8 n^{2}-9\right)^{1 / 2} / 4\right) \Gamma\left(3 / 4-i\left(8 n^{2}-9\right)^{1 / 2} / 4\right)},
\end{aligned}
$$

together with the asymptotic expansion of the $\Gamma$-function $\left({ }^{48}\right)$, from which we may show that

$$
\begin{aligned}
& \Gamma(x+i y) \Gamma(x-i y) \sim 2 \pi \exp [-2 x-2 y \arctan (y / x)]\left(x^{2}+y^{2}\right)^{x-1 / 2} \\
& \quad \cdot\left\{1+289 x /\left(1728\left[x^{2}+y^{2}\right]\right)+\left(173 x^{2}+259 y^{2}\right) /\left(31104\left[x^{2}+y^{2}\right]^{2}\right)+\cdots\right\},
\end{aligned}
$$

enables us to derive an asymptotic formula which serves for ready computations :

$$
\begin{aligned}
& F\left(\left[3+i\left(8 n^{2}-9\right)^{1 / 2}\right] / 2,\left[3-i\left(8 n^{2}-9\right)^{1 / 2}\right] / 2 ; 2 ; 1 / 2\right) \\
& \approx 2^{-3 / 4}(n \pi)^{-1 / 2} \exp \left[3 / 2+\left(\left[8 n^{2}-9\right]^{1 / 2}\right) / 2 \cdot \arctan \left(\left[8 n^{2}-9\right]^{1 / 2} / 3\right)\right] .
\end{aligned}
$$

The values of this function for $n=8,16, \cdots$ are $5.36 \times 10^{6}, 1.31 \times 10^{14}, \cdots$. But even when $z>a / 2$, that is, in the upper half of the dome, the remaining hypergeometric series occurring in this example converge so slowly as to make computation impractical unless some asymptotic formula is used. Instead of attempting to complete this example numerically, we shall consider a dome of a very similar meridian for which the computations are much easier. In passing we need merely notice that at the apex $z=a$ the hypergeometric functions in the numerator of (6) have the value 1 , while at the base $z=0$ they are very large; thus the effect of the singularity at the apex will be confined to a very small region near it.

A shell whose meridian is practically indistinguishable from (1) (see Fig. 17 ) is given by the formula

$$
f=A \cos [\pi z /(2 a)] .
$$

The solution for the uniformly supported shell loaded only by its own weight is

$$
\begin{aligned}
& N_{\phi 0}=-\frac{4 a p}{3 \pi} \frac{\left\{1+\left[A^{2} \pi^{2} /\left(4 a^{2}\right)\right] \sin ^{2}[\pi z /(2 a)]\right\}^{1 / 2}}{\cos [\pi z /(2 a)]} \\
& \text { - }\left[\left\{1+A^{2} \pi^{2} /\left(4 a^{2}\right)\right\}^{3 / 2}-\left\{1+\left[A^{2} \pi^{2} /\left(4 a^{2}\right)\right] \sin ^{2}[\pi z /(2 a)]\right\}^{3 / 2}\right], \\
& N_{\theta 0}=-\left[p A^{2} \pi /(4 a)\right] \sin [\pi z / a] \\
& -\frac{A^{2} \pi^{2}(\cos [\pi z / a]+1)}{8 a^{2}\left\{1+\left[A^{2} \pi^{2} /\left(4 a^{2}\right)\right] \sin ^{2}[\pi z /(2 a)]\right\}} N_{\phi} .
\end{aligned}
$$

The solution for the shell supported on $m$ columns is easily found from the results (21.5):

(48) Whittaker and Watson (1927), p. 253. 
(9)

$$
\begin{aligned}
& N_{\phi}=-\frac{2 P\left(1+\left[A^{2} \pi^{2} /\left(4 a^{2}\right)\right] \sin ^{2}[\pi z /(2 a)]\right)^{1 / 2}}{\epsilon \cos ^{2}[\pi z /(2 a)]} \\
& \text {. } \sum_{n=m, 2 m, \ldots}^{\infty} \frac{\sin n \epsilon}{n} \frac{\operatorname{sh}\left[\pi\left(n^{2}-1\right)^{1 / 2}(a-z) /(2 a)\right]}{\operatorname{sh}\left[\pi\left(n^{2}-1\right)^{1 / 2} / 2\right]} \cos n \theta, \\
& N_{\theta}=-\frac{\pi^{2} A^{2} \cos ^{2}[\pi z /(2 a)]}{4 a^{2}\left\{1+\left[A^{2} \pi^{2} /\left(4 a^{2}\right)\right] \sin ^{2}[\pi z /(2 a)]\right\}} N_{\phi} \text {, } \\
& N_{\theta \phi}=\frac{P A \pi}{a \epsilon} \sec [\pi z /(2 a)]\{\tan [\pi z /(2 a)] \\
& \text {. } \sum_{n=m, 2 m, \ldots}^{\infty} \frac{\sin n \epsilon}{n} \frac{\operatorname{sh}\left[\pi\left(n^{2}-1\right)^{1 / 2}(a-z) /(2 a)\right]}{\operatorname{sh}\left[\pi\left(n^{2}-1\right)^{1 / 2} / 2\right]} \sin n \theta \\
& \left.-\sum_{n=m, 2 m, \ldots}^{\infty} \frac{\left(n^{2}-1\right)^{1 / 2}}{n} \sin n \epsilon \frac{\operatorname{ch}\left[\pi\left(n^{2}-1\right)^{1 / 2}(a-z) /(2 a)\right]}{\operatorname{sh}\left[\pi\left(n^{2}-1\right)^{1 / 2} / 2\right]} \sin n \theta\right\} .
\end{aligned}
$$

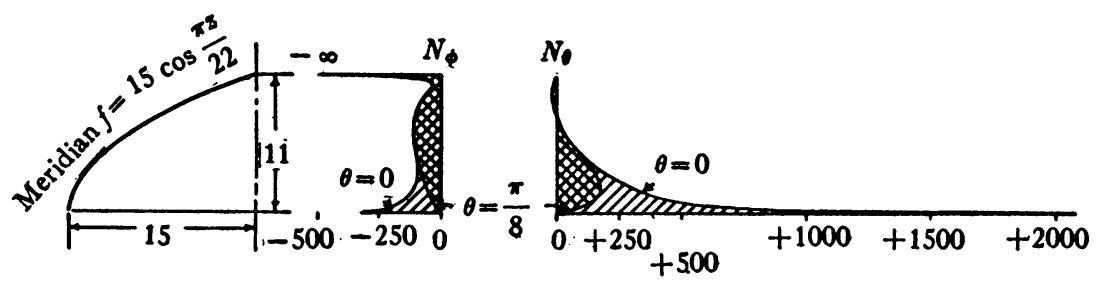

FIG. 18.

For comparison with Flügge's numerical results for the meridian (1), I have appended a graph (Fig. 18) of the solutions (8) and (9) superposed, when $p=1, a=11, A=15, \epsilon=\pi / 64, m=8$. The results are qualitatively the same as Flügge's, except that in his work, as we said before, the fact that $N_{\phi}$ is infinite at the apex does not appear. The quantitative difference may be due to three reasons: that Flügge's results are approximate, that Flügge does not state, and indeed does not seem to use, the width of the supports, and that the meridian (7) is slightly different from the meridian (1). The effect of the nonuniform support is noticeable only in the lower half of the dome, and in that there is a singularity, quite unperceivable except in a small region near the apex.

25. Two open domes. The dome whose meridian is the hyperbola (see Fig. 19)

$$
f=A / z
$$


is free of singularity in the region where $z>0$. Let us first treat a shell supported uniformly at $z=z_{0}$ bearing uniformly a load $H$, say the weight of some

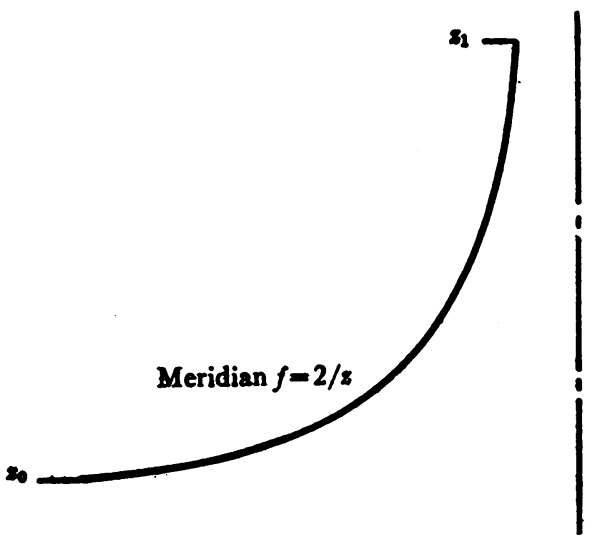

FIG. 19.

superstructure, at $z=z_{1}$. Then we shall prescribe that $\left.N_{\phi 0}\right|_{z-z 1}$ shall be the resultant of the downward force/length due to the load, $-\left.H\left(2 \pi r_{2} \sin \phi\right)\right|_{z-z_{1}}$, and a horizontal force/length due to a stiffener:

$$
\left.N_{\phi 0}\right|_{z=z_{1}}=-H\left(A^{2}+z_{1}^{4}\right)^{1 / 2} /\left(2 \pi A z_{1}\right) .
$$

From the integral (12.4) the desired solution is easily found.

$$
\begin{aligned}
N_{\phi 0}=\frac{p\left(A^{2}+z^{4}\right)^{1 / 2}}{2 z}\left[\frac{\left(A^{2}+z^{4}\right)^{1 / 2}}{z^{2}}-\right. & \frac{\left(A^{2}+z_{1}^{4}\right)^{1 / 2}}{z_{1}^{2}} \\
& \left.-\log \frac{z^{2}+\left(A^{2}+z^{4}\right)^{1 / 2}}{z_{1}^{2}+\left(A^{2}+z_{1}^{4}\right)^{1 / 2}}-\frac{H}{\pi a p}\right] .
\end{aligned}
$$

To solve unsymmetrical problems we shall need the real form of the solution (21.9),

$$
\mathfrak{U}_{n c}=A_{n} z^{1 / 2} \sin \left[\frac{\left(8 n^{2}-9\right)^{1 / 2}}{2} \log \frac{z}{B_{n}}\right] .
$$

Suppose now the shell bears its load $H$ at $z=z_{1}$ on $r$ symmetrical columns of angular width $2 \eta$, and is itself supported at $z=z_{0}$ on $s$ symmetrical columns of angular width $2 \epsilon, \theta=0$ being the location of the center of one column of each set; then upon (3) must be superposed the solution 


$$
N_{\phi}=z^{1 / 2}\left(A^{2}+z^{4}\right)^{1 / 2}\left\{\frac{2 P}{\eta z_{1}^{1 / 2}\left(A^{2}+z_{1}^{4}\right)^{1 / 2}}\right.
$$

(5)

$$
\times \sum_{n=r, 2 r, \ldots}^{\infty} \frac{\sin n \epsilon}{n} \frac{\sin \left[\frac{\left(8 n^{2}-9\right)^{1 / 2}}{2} \log \frac{z}{z_{0}}\right]}{\sin \left[\frac{\left(8 n^{2}-9\right)^{1 / 2}}{2} \log \frac{z_{1}}{z_{0}}\right]} \cos n \theta
$$

$$
+\frac{2 Q}{\epsilon z_{0}^{1 / 2}\left(A^{2}+z_{0}^{4}\right)^{1 / 2}}
$$

$$
\left.\times \sum_{n=s, 2 s, \ldots}^{\infty} \frac{\sin n \epsilon}{n} \frac{\sin \left[\frac{\left(8 n^{2}-9\right)^{1 / 2}}{2} \log \frac{z}{z_{1}}\right]}{\sin \left[\frac{\left(8 n^{2}-9\right)^{1 / 2}}{2} \log \frac{z_{0}}{z_{1}}\right]} \cos n \theta\right\}
$$

where

$$
P=-\left.N_{\phi 0}\right|_{z=\varepsilon_{1}}, \quad Q=-\left.N_{\phi 0}\right|_{z-\varepsilon_{0}},
$$

$N_{\phi 0}$ being given by (3).

If the meridian is of form

$$
f=a+b z^{\nu}
$$

points on the ring $z=0$ are singularities, and the considerations of $\$ 17$ are applicable. It is not difficult to verify that

$$
\mathfrak{U}_{n 1}=z F\left((1+p) /(2 v),(1-p) /(2 v) ; 1+1 / \nu ;-b z^{\nu} / a\right),
$$

where $p \equiv\left[1-4 \nu(\nu-1)\left(n^{2}-1\right)\right]^{1 / 2}$. The case when $\nu=1 / 2$,

$$
f=a+b z^{1 / 2} \text {, }
$$

(see Fig. 20) is of particular interest; for this case

$$
\mathfrak{u}_{n 1}=z F\left(1+n, 1-n ; 3 ;-b z^{1 / 2} / a\right)=z H_{n-1}\left(-b z^{1 / 2} / a\right),
$$

where $H_{n}(x)$ is Jacobi's polynomial $\left({ }^{49}\right)$. For a profile of $N_{\phi n}{ }^{(1)}$ see Fig. 20, with which Fig. $8, \S 21$, should be compared, the meridians being of the same shape but the one shell having a closed top, the other an aperture. All sorts of unsymmetrical problems are easily solved with the meridian (9).

(49) Bateman (1932), p. 392. 

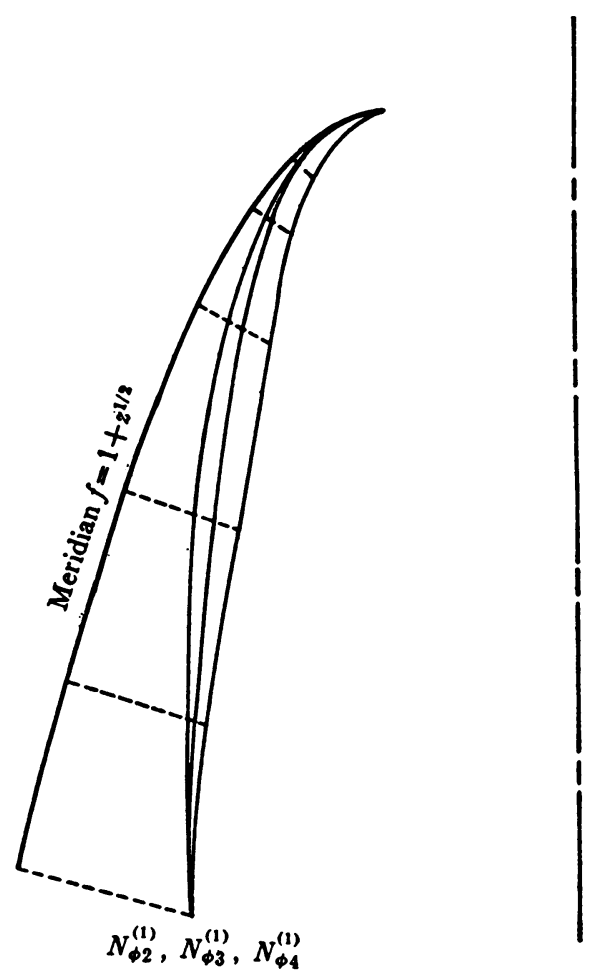

FIg. 20.

26. Two domes with a point of inflection in the meridian. The meridian

$$
f=A z^{\mu}(1+B z)
$$

has a point of inflection at the point $z=-A(\mu-1) /(B(\mu+1))$. The stress functions satisfying the boundary conditions at $z=0$, if they can be satisfied at all, are given by the formula

$$
\mathfrak{U}_{n 1}=z^{(p+1) / 2} F(a, b ; c ;-B z),
$$

where

$$
\begin{aligned}
& p \equiv\left[1-4 \mu(\mu-1)\left(n^{2}-1\right)\right]^{1 / 2}, \quad a \equiv(p+q) / 2, \quad b \equiv(p-q) / 2, \\
& q \equiv\left[1-4 \mu(\mu+1)\left(n^{2}-1\right)\right]^{1 / 2}, \quad c \equiv 1+p .
\end{aligned}
$$

When $\mu=1 / 2$ the formula (2) becomes simply

(3) $\mathfrak{U}_{n 1}=z^{(n+1) / 2} F\left(n / 2+i\left(3 n^{2}-4\right)^{1 / 2} / 2 ; n / 2-i\left(3 n^{2}-4\right)^{1 / 2} / 2 ; n+1 ;-B z\right)$.

If one sketches a curve of the family (1), he will find the point of inflection 
hardly noticeable, as may be seen from the special case for which we give a profile in Fig. 21.

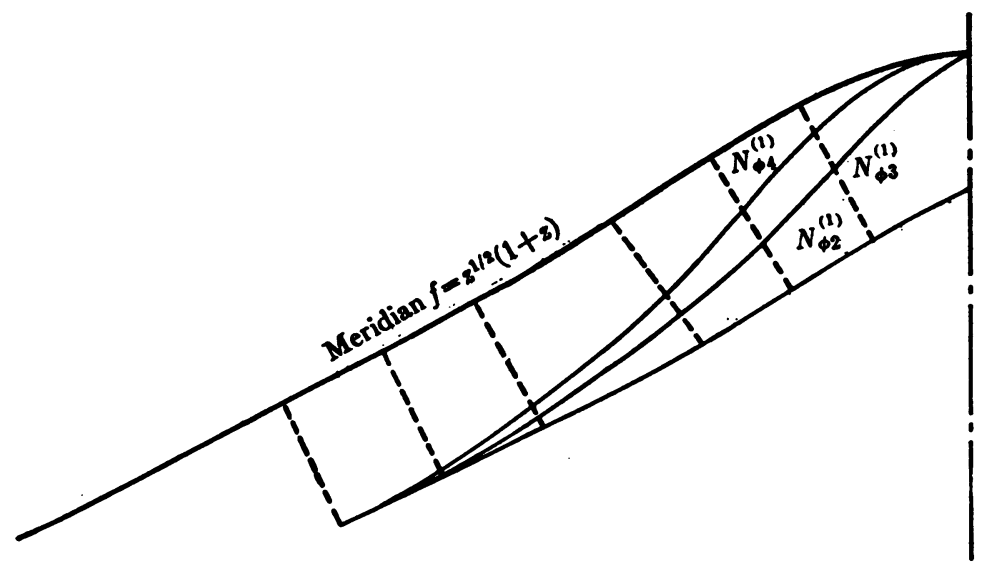

FIG. 21.

A meridian with a much more marked point of inflection is given by (4)

$$
f=(\tan z)^{1 / 2} \text {. }
$$

For this meridian, the complete stress functions satisfying the boundary conditions at the apex $z=0$ are given by the formula

$$
\begin{aligned}
\mathfrak{U}_{n 1}=\tan ^{(n+1) / 2} z F\left(\left[n+2+i\left(3 n^{2}-4\right)^{1 / 2}\right] / 4,\right. \\
\\
{\left.\left[n+2-i\left(3 n^{2}-4\right)^{1 / 2}\right] / 4 ; n / 2+1 ;-\tan ^{2} z\right) . }
\end{aligned}
$$

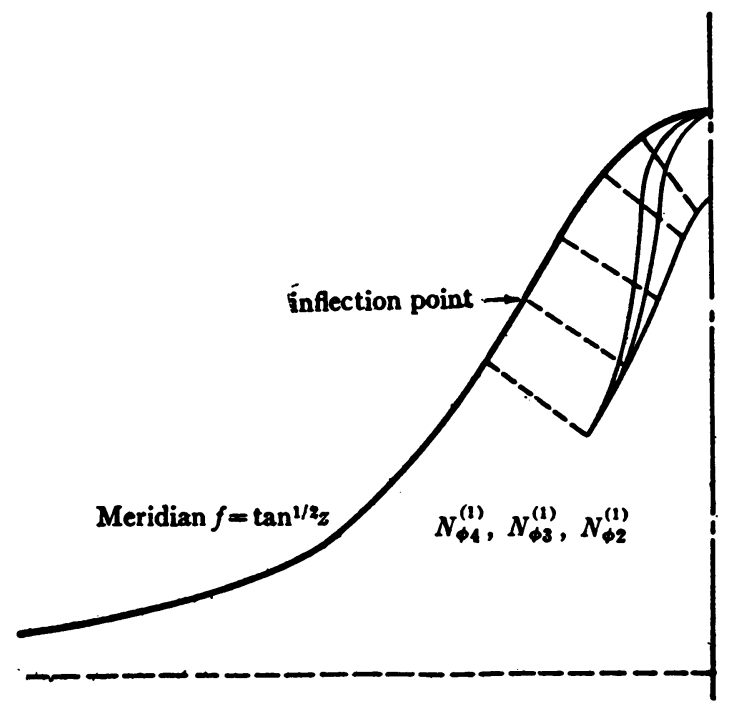

FIG. 22. 
Unlike the series occurring in (24.6), the hypergeometric series (5) converge very rapidly even reasonably far from the apex, provided $n$ is not too large, and the profile (Fig. 22) was not very tedious to compute. Since the exponent of (4) at the apex is $1 / 2$ (see $\$ 15$ ), all sorts of problems may be treated successfully for this meridian; the labor of computation is much less than for the meridian (1).

27. Stress functions for a few other surfaces. To save possible labor for engineers computing shells we list a few other meridians and their stress functions.

If $c \equiv-1 / 2+\left(1-A^{2}\right)^{1 / 2} / 2$, and if $\mathbb{R}_{n}^{m}[x]$ is the complete solution of the associated Legendre equation, then the stress functions $\mathfrak{u}_{n c}$ for the family of surfaces with meridian

$$
f=\mathfrak{R}_{c}^{A b}[\operatorname{tnh}(z+a)]
$$

may be obtained by replacing $A^{2}$ by $-A^{2}\left(n^{2}-1\right)$.

$$
\begin{aligned}
f & =A\left(e^{z}-e^{b}\right) \\
\mathfrak{U}_{n 1} & =F\left(i\left(n^{2}-1\right)^{1 / 2},-i\left(n^{2}-1\right)^{1 / 2} ; 1 ; e^{z-b}\right) . \\
f & =(\sin z)^{1 / 2}
\end{aligned}
$$

(3) $\mathfrak{U}_{n 1}=\sin ^{(n+1) / 2} z F\left(\left[n+1+i\left(n^{2}-1\right)^{1 / 2}\right] / 4\right.$,

$$
\left.\left[n+1-i\left(n^{2}-1\right)^{1 / 2}\right] / 4 ; n+1 / 2 ; \sin ^{2} z\right) \text {. }
$$

$$
f=A \tan z
$$

$$
\mathfrak{U}_{n 1}=F\left(a, \bar{a} ; 1 ; \sec ^{2} z\right)
$$

$$
\begin{aligned}
= & \frac{\pi^{1 / 2}}{\Gamma(b) \Gamma(\bar{b})} F\left(a, \bar{a} ; 1 / 2 ;-\tan ^{2} z\right) \\
& +\frac{2 \pi^{1 / 2} \tan z}{\Gamma(a) \Gamma(\bar{a})} F\left(b, b ; 3 / 2 ;-\tan ^{2} z\right),
\end{aligned}
$$

where $a \equiv\left[1+i\left(8 n^{2}-9\right)^{1 / 2}\right] / 4, b \equiv a+1 / 2$.

$$
\begin{aligned}
f & =A \sin ^{2} z, \\
\mathfrak{U}_{n 1} & =\sin ^{p} z F\left(-1,0 ; p+2 i\left(n^{2}-1\right)^{1 / 2}, p-2 i\left(n^{2}-1\right)^{1 / 2}, 2 p, 1 / 2 ; \sin z\right),
\end{aligned}
$$

where $p \equiv-1 / 2-i\left(8 n^{2}-5\right)^{1 / 2} / 2$, and $F(a, q ; \alpha, \beta, \gamma, \delta ; x)$ is the function of Heun $\left({ }^{50}\right)$. This last meridian is an example of Case III, $\$ 15$, and hence, as we may verify from the stress functions themselves, is not suitable for problems of shell theory unless the apex is cut out. Should one wish to treat it, a more convenient solution can be obtained in the form

(50) Whittaker and Watson (1927), pp. 576-577, exercises 7-10 


$$
\mathfrak{u}_{n c}=a_{0}+\sum_{m=1}^{\infty}\left(a_{m} \cos 2 m z+b_{m} \sin 2 m z\right),
$$

where $a_{1}=0, a_{2}=2\left(n^{2}-1\right) a_{0} /\left(n^{2}+3\right), b_{2}=2 b_{1} /\left(n^{2}+3\right)$, and both the $a_{n}$ and the $b_{n}$ satisfy the same recurrence formula

$$
\left(m^{2}+2 m+n^{2}\right) a_{m+1}=2 m^{2} a_{m}-\left(m^{2}-2 m+n^{2}\right) a_{m-1} .
$$

28. Nemenyi's integral equation. If we integrate the equation of the stress functions (11.8) twice, by reversing the order of integrations we obtain a linear integral equation satisfied by the function $\mathfrak{u}_{n}$ :

$$
\begin{aligned}
\mathfrak{U}_{n}(z)= & \mathfrak{U}_{n}(a)+(z-a) \mathfrak{U}_{n}^{\prime}(a) \\
& +\left(1-n^{2}\right) \int_{a}^{z} d \xi(z-\xi) \frac{f^{\prime \prime}(\xi)}{f(\xi)} \mathfrak{U}_{n}(\xi)+\int_{a}^{z} d \xi \int_{a}^{\xi} g_{n}(\eta) d \eta
\end{aligned}
$$

Since by differentiating (1) we may obtain (11.8), (1) is an equivalent form of the stress equation. If it is possible to satisfy the conditions of equilibrium at the top of a closed dome, $z=a$, we shall find that $\mathfrak{U}_{n}(a)=0$, and usually $\mathfrak{U}_{n}^{\prime}:(a)=0$. Then for the homogeneous stress functions $\mathfrak{U}_{n c}$ the equation (1) becomes the integral equation

$$
\mathfrak{u}_{n c}(z)=\left(1-n^{2}\right) \int_{a}^{z}(z-\xi) \frac{f^{\prime \prime}(\xi)}{f(\xi)} \mathfrak{u}_{n c}(\xi) d \xi,
$$

originally deduced by Nemenyi $\left.{ }^{51}\right)$ from graphostatic considerations. It was through this equation that Nemenyi introduced stress functions which are essentially the $\mathfrak{U}_{n c}$ and arrived at a special case of (11.8). Nemenyi has given a simple physical interpretation of (2); the right-hand side represents the static moment of a section of the meridian line of the middle section from the apex $z=a$ to $z$, subject to a load $f^{\prime \prime} \mathfrak{U}_{n c} / f$. Then if one has a start at the apex, one may proceed in finite steps downward, computing approximate values of $\mathfrak{U}_{n c}$ by graphical means. To obtain $\mathfrak{u}_{n}$ when there is a load, one need only add the integral involving $g_{n}(z)$ in (1), which may be computed mechanically if necessary. To obtain the start at the apex, one may approximate the middle section either by the curve $f=A z^{\mu}$ or by the curve $f=A z^{\mu}(1+B z)$ which we have treated exactly in the previous sections (see (21.9) and (26.1) respectively). Thus the stresses for any load distribution on any dome may be found approximately.

While the graphical means just outlined may serve to treat a quite arbitrary meridian, it is tedious to carry out. A more practical approach, probably, would be, given a meridian, to find one approximating it closely from one of the numerous infinite families which we found in the previous sections to be amenable to a simple exact solution.

( $\left.{ }^{51}\right)$ Nemenyi (1936). 


\section{INDEX OF DEFINITIONS AND NOTATIONS}

Only those quantities used in more than one section are listed. The numbers refer to the section where the quantity or term is defined.

complete stress functions

conical coordinates

displacement functions

insoluble

membrane stress

moment resultants

particular stress functions

revolution coordinates

singularity (of a shell)

soluble

soluble in the membrane theory

stress functions

stress resultants

thickness

$A(\theta), B(\theta)$

$C_{n}, D_{n}$

$D$

$E$

$E_{n}, F_{n}$

$F$

$f, f(z)$

$g_{n}(z)$

$h_{n}(z)$

$K$

$L_{x}, L_{y}$

$M_{\theta}, M_{\phi}, M_{\theta \phi}, M_{\phi \theta}, M_{y}, M_{\theta y}, M_{y \theta}$
$11 N_{\theta}, N_{\phi}, N_{y \theta}, N_{\theta y}, N_{\theta \phi}, N_{\phi \theta}$

$3 N_{\theta n}, N_{\phi n}, N_{\theta \phi n}$

$18 N_{\phi n}^{(1)}$

$9 p$

$6,10 Q_{\phi}, Q_{\theta}, Q_{\nu}$

$4 \quad R$

$11 r_{1}, r_{2}$

$3 u, v, w$

$3 U, V, W$

$9 U_{n}, V_{n}, W_{n}$

$15 \mathfrak{U}_{n}, \mathfrak{U}_{n 1}, \mathfrak{U}_{n 2}, \mathfrak{U}_{n p}, \mathfrak{U}_{n c}$

$11 \mathfrak{B}_{n}, \mathfrak{B}_{n 1}, \mathfrak{B}_{n 2}, \mathfrak{B}_{n p}$

$4 x$

$3 X, Y, Z$

$X_{n}, Y_{n}, Z_{n}$

$6 X_{t}, Y_{t}, Z_{t}$

$11 y$

5 z

5

$18 \alpha$

$14,7 \quad \alpha_{n 1}, \alpha_{n 2}$

$8 \delta$

$11 \Delta$

$18 \theta$

$5 \mu$

$4 \nu$

$4 \phi$
$3,5,9$

3

5,8

5,8

18

11

18

3

6,10

11

4

3

3

15

3

5

3

15

5

3

\section{REFERENCES}

This bibliography lists only those books and memoirs to which the text refers explicitly.

\section{THREE-DIMENSIONAL ELASTICITY}

\section{Books}

A. E. H. Love, The mathematical theory of elasticity, 4th ed., Cambridge, 1927.

E. Trefftz, Mathematische Elastizitätstheorie, Handbuch der Physik, vol. 6, 1928, Berlin.

I. S. Sokolnikoff, Mathematical theory of elasticity (notes), Brown University, 1941. MEMOIRS

G. Lamé, Memoire sur l'équilibre d'élasticité des enveloppes spheriques, Journal des Mathematiques vol. 19 (1854) pp. 51-87. 
H. Neuber, Ein neuer Ansatz zur Lösung räumlicher Probleme der Elastizitätstheorie, Zeitschrift für Angewandte Mathematik und Mechanik vol. 14 (1934) pp. 203-212.

F. Odqvist, Equations de compatibilité pour une système de coordonnés triples orthogonaux quelconques, C. R. Acad. Sci. Paris vol. 205 (1937) pp. 202-204.

\section{Books}

THE THEORY OF SHELLS

J. W. Geckeler, Elastostatik, Handbuch der Physik, vol. 6, 1928, Berlin.

W. Flügge, Statik und Dynamik der Schalen, Berlin, 1934.

S. Timoshenko, Theory of plates and shells, New York and London, 1940. MEMOIRS

H. Aron, Das Gleichgewicht und die Bewegung einer unendlich dïnnen, beliebig gekrimmten elastischen Schale, Math. Ann. vol. 78 (1874) pp. 136-174. 1912.

H. Reissner, Spannungen in Kugelschalen (Kuppeln), Müller-Breslau Festschrift, Leipzig,

E. Schwerin, Über die Spannungen in symmetrisch und unsymmetrisch belasteten Kugeln (Kuppeln) usw., Dissertation (1917), Berlin, 1918. Berlin.

F. Dischinger, Schalen und Rippenkuppeln, Handbuch für Eisenbetonbau, vol. 6, 1928,

P. Nemenyi, Beiträge zur Berechnung der Schalen unter unsymmetrischer und unstetiger Belastung, Bygningsstatiske Meddelelser (Denmark), 1936.

A. Pucher, Über die Spannungsfunktion beliebig gekrümmter dünner Schalen, Proceedings of the Fif th International Congress of Applied Mechanics, New York, 1939.

E. Reissner, $A$ new derivation of the equations for the deformation of elastic shells, Amer. J. Math. vol. 63 (1941) pp. 177-184.

J. L. Synge and W. Z. Chien, Intrinsic theory of elastic shells and plates, Karman Anniversary Volume, Pasadena, 1941.

P. Nemenyi and C. Truesdell, $A$ stress function for the membrane theory of shells of revolution, Proc. Nat. Acad. Sci. U.S.A. vol. 29 (1943) pp. 159-162.

C. Truesdell, The membrane theory of shells of revolution, Dissertation (1943), MS in Princeton Library.

\section{Differential EQUATIONS AND SPECIAL FUNCTIONS}

E. T. Whittaker and G. N. Watson, $A$ course of modern analysis, 4 th ed., Cambridge, 1927.

H. Bateman, Partial differential equations of mathematical physics, Cambridge, 1932.

\section{Princeton University,}

Princeton, N. J. 